Development of Ni-based Oxide Dispersion Strengthened (ODS) powder particles for Metal Additive Manufacturing (AM) Applications

\author{
Markova Dion McPherson \\ mdmcpherson@mix.wvu.edu
}

Follow this and additional works at: https://researchrepository.wvu.edu/etd

\author{
Recommended Citation \\ McPherson, Markova Dion, "Development of Ni-based Oxide Dispersion Strengthened (ODS) powder \\ particles for Metal Additive Manufacturing (AM) Applications" (2019). Graduate Theses, Dissertations, \\ and Problem Reports. 4027. \\ https://researchrepository.wvu.edu/etd/4027
}

This Thesis is protected by copyright and/or related rights. It has been brought to you by the The Research Repository @ WVU with permission from the rights-holder(s). You are free to use this Thesis in any way that is permitted by the copyright and related rights legislation that applies to your use. For other uses you must obtain permission from the rights-holder(s) directly, unless additional rights are indicated by a Creative Commons license in the record and/ or on the work itself. This Thesis has been accepted for inclusion in WVU Graduate Theses, Dissertations, and Problem Reports collection by an authorized administrator of The Research Repository @ WVU. For more information, please contact researchrepository@mail.wvu.edu. 


\title{
Development of Ni-based Oxide Dispersion Strengthened (ODS) powder for Metal Additive Manufacturing (AM) Applications
}

\author{
Markova Dion McPherson
}

\author{
Thesis submitted to the \\ College of Engineering and Mineral Resources \\ West Virginia University \\ In partial fulfilment of the requirements \\ For the degree of
}

\author{
Master of Science \\ In \\ Mechanical Engineering
}
Bruce Kang, Ph.D. Committee Chairman
Kenneth H. Means, Ph.D. Jacky C. Prucz, Ph.D.

\section{Department of Mechanical and Aerospace Engineering Morgantown, West Virginia}

2019

Keywords: Oxide-Dispersion, ODS alloy, Mechanical Alloying, Additive Manufacturing Copyright 2019 Markova D. McPherson 


\section{ABSTRACT \\ Development of Ni-based Oxide Dispersion Strengthened (ODS) powder for Metal Additive Manufacturing (AM) Applications}

\section{Markova Dion McPherson}

The capabilities of Additive Manufacturing (AM) techniques have grown rapidly in recent years, however, current available metal powders for AM processes, such as Powder Bed Fusion and Directed Energy Deposition, are limited and primarily fabricated through gas atomization process which; the gas atomization process is capable of producing metal powders $15 \mu \mathrm{m}$ to $150 \mu \mathrm{m}$ in size with near spherical shape. Despite the advantages of atomization process, iron or nickel-based Oxide Dispersion Strengthened (ODS) powders, with nanocrystalline microstructure, cannot be produced with the gas atomization process because of the high melting temperature of yttrium (III) oxide $\left(\mathrm{Y}_{2} \mathrm{O}_{3}\right.$, $\left.2425^{\circ} \mathrm{C}\right)$ compared to iron $\left(\mathrm{Fe}, 1538^{\circ} \mathrm{C}\right)$, nickel $\left(\mathrm{Ni}, 1668^{\circ} \mathrm{C}\right)$, chromium $\left(\mathrm{Cr}, 1907^{\circ} \mathrm{C}\right)$ and aluminum ( $\mathrm{Al}, 660^{\circ} \mathrm{C}$ ), thus, uniform dispersion of $\mathrm{Y}_{2} \mathrm{O}_{3}$ is problematic for ODS powders. In this work, a combination of Mechano-Chemical Bonding (MCB) process and Mechanical Alloying (MA) by planetary ball milling (BM) will be implemented to produce ODS powders suitable for AM applications. The MCB process fractures and uniformly disperse the $\mathrm{Y}_{2} \mathrm{O}_{3}$ nanoparticles and the nanoparticles are bonded on the surface of the master particles ( $\mathrm{Ni}$ and $\mathrm{Cr}$ ). Also, the MA process, because of the constant fracturing and cold-welding of the elemental particles, produces alloyed ODS powders with suitable uniform size distribution, near spherical shape, and nanocrystalline microstructure.

The objectives of this research are to (1) optimize the MCB+BM processing parameters and (2) study effects of the process parameters on the size, morphology, and microstructure of Ni-based ODS powders for metal AM applications using Laser Engineering Net Shaping (LENS) machine.

Results showed that Ni-based ODS particles with nearly spherical in morphology, average particle size of $15 \mu \mathrm{m}$, uniform dispersion of $\mathrm{Y}_{2} \mathrm{O}_{3}$, and nanocrystalline microstructure can be successfully produced via the proposed $\mathrm{MCB}+\mathrm{BM}$ methodology. These resultant Ni-based ODS particles were successfully used on a LENS AM machine to produce coupon specimen. The coupon specimen microstructure contains $\mathrm{Y}-\mathrm{NiAl}$ matrix and submicron $\mathrm{y}^{\prime}-\mathrm{Ni}_{3} \mathrm{Al}$ strengthening phase. 


\section{ACKNOWLEDGEMENTS}

Thanks you to Dr. Bruce Kang, my academic advisor, for his guidance and support for my research efforts to obtain my Master's Degree. I am also thankful for Dr. Kenneth $\mathrm{H}$. Means and Dr. Jacky $\mathrm{C}$. Prucz for suggestions, advice, and criticism given of my research.

I am also grateful to the MAE department, especially Dr. Victor Mucino, Pam Gelet, and Pat Goldie, for the opportunity to work as a Teaching Assistant and Research Assistant during my graduate studies.

This work would not have been possible without help from members in our research group: Jaeyoon Kim, Eric Chia, Sizhe Huang, and Changyu Ma. Without the combined efforts of their research, help, discussion, and time, I could not finish this study.

I will also like to thank my mentors Robert O. Lampl, Jonathan Ferguson, and John Batton for their consistent support and guidance.

Thanks and definitely not last, I have a special thanks and love for my mother and older sister for their continuous love and support when I needed it the most.

I am thankful and grateful for all the motivation, advice, guidance, and support given to me. 


\section{TABLE OF CONTENTS}

ABSTRACT

ACKNOWLEDGEMENTS

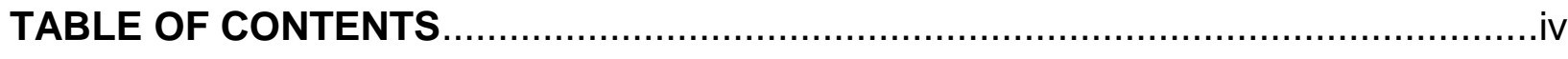

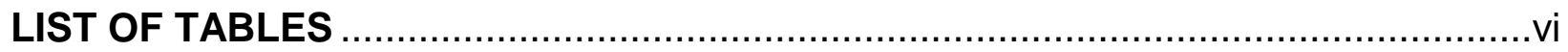

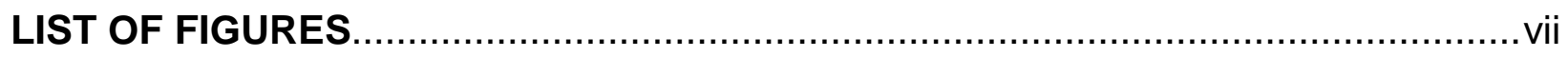

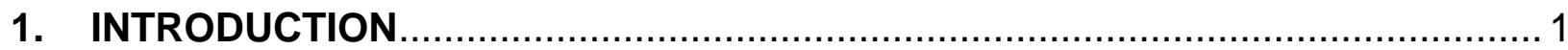

1.1 Metal Powder Additive Manufacturing ............................................... 1

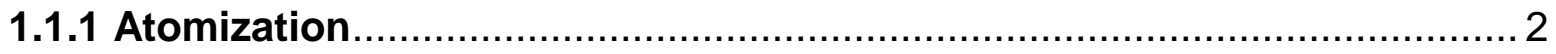

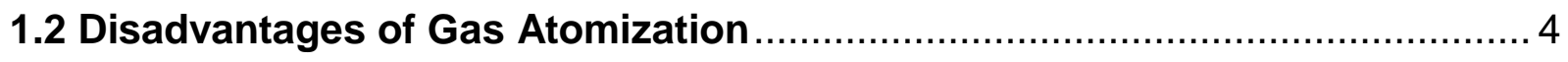

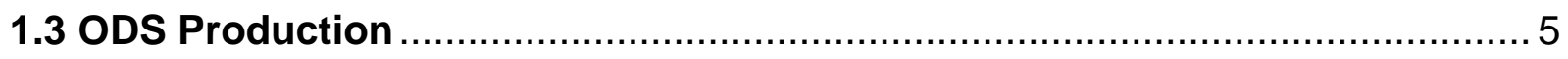

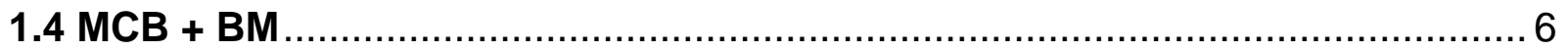

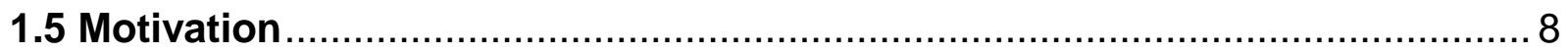

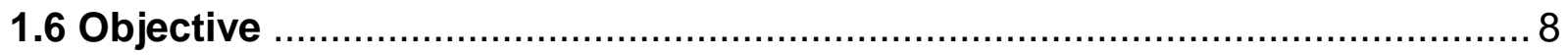

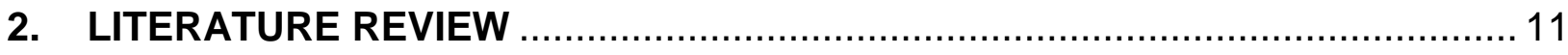

2.1 Superalloys and Oxide Dispersion Strengthened (ODS) Superalloys .......... 11

2.1.1 ODS Ni-based Superalloys ............................................................. 12

2.2 Fabrication Techniques of ODS alloy Powders ...................................... 13

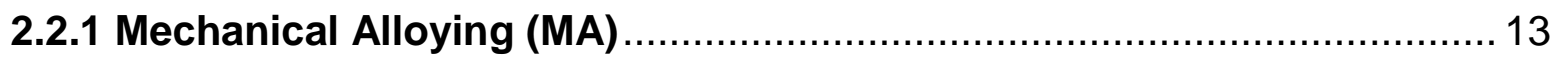

2.2.2 Mechano-Chemical Bonding (MCB) …......................................... 15

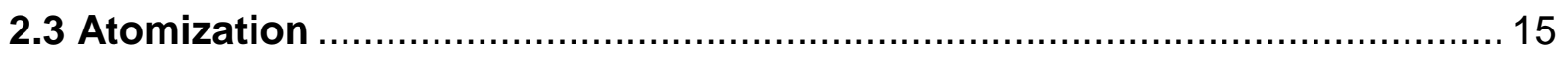

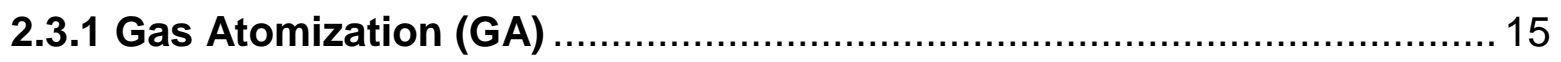

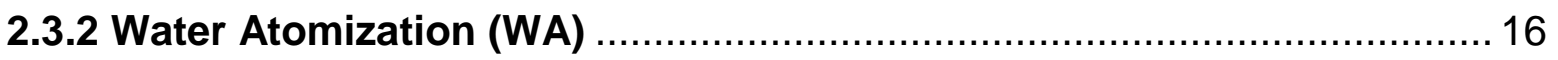

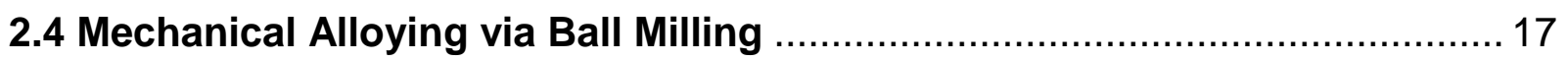

2.4.1 Parameters of Planetary Ball Milling ................................................. 17

2.4.2 Parameter Process Controls of Ball Milling ......................................... 18

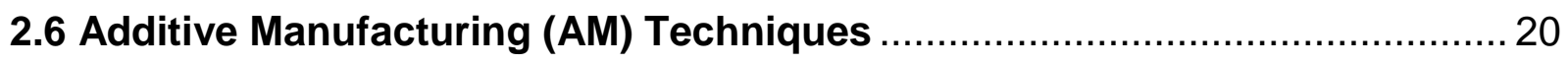

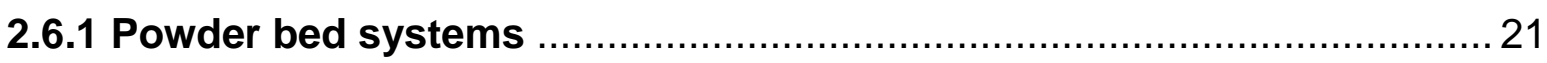

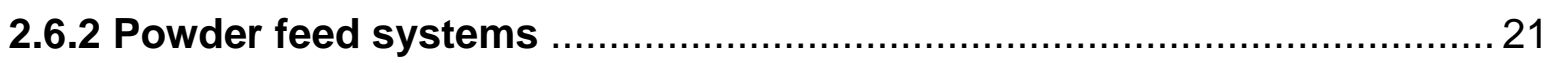

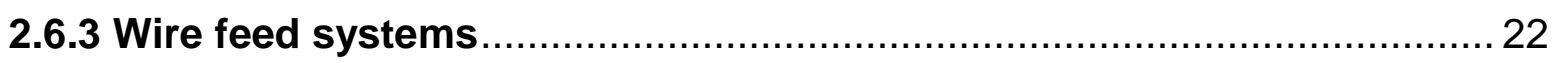

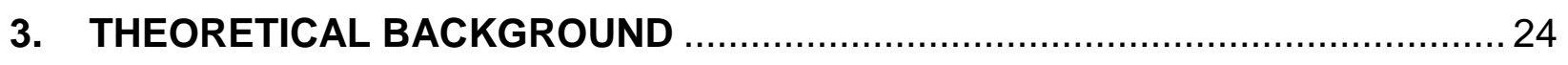

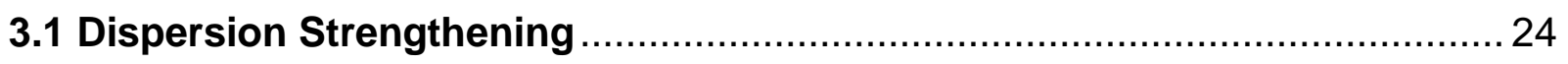

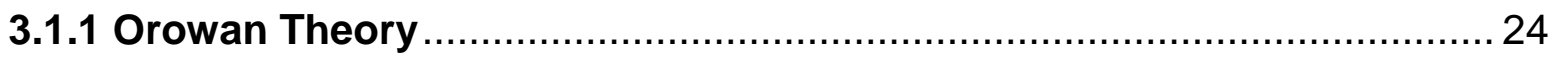




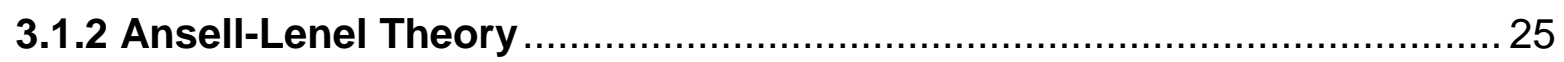

3.2 Elemental composition and effects on ODS Ni-based Superalloys ............. 25

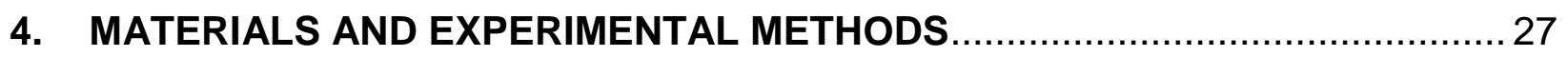

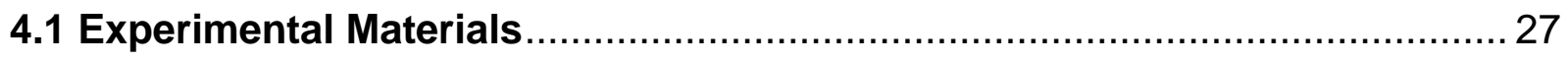

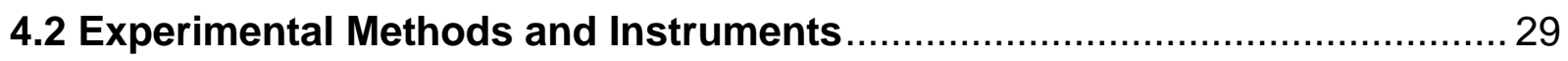

4.2.1 Mechano-Chemical Bonding (MCB) Process........................................... 29

4.2.2 Mechanical Alloying via Ball Milling (BM) .......................................... 29

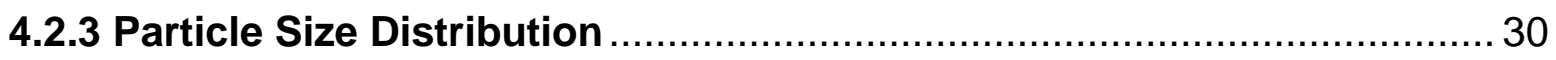

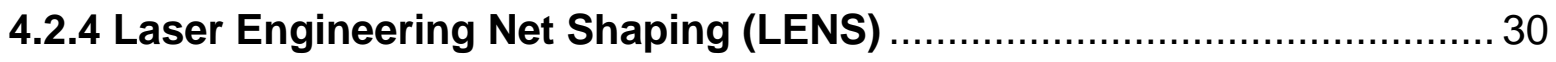

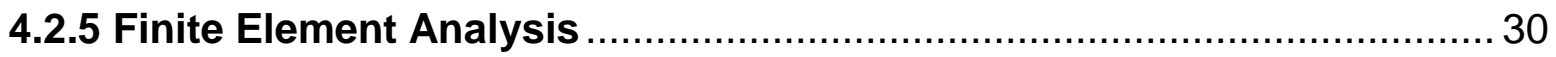

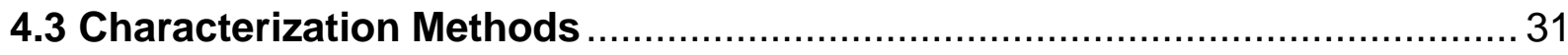

4.3.1 SEM

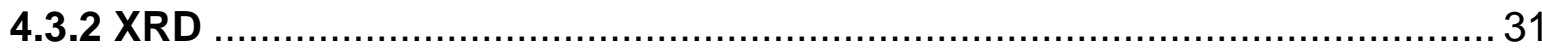

4.3.3 EDS

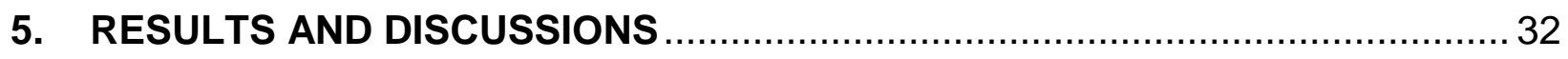

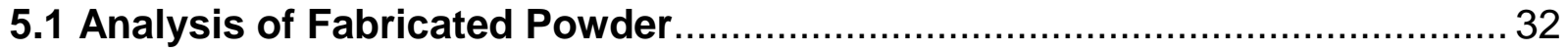

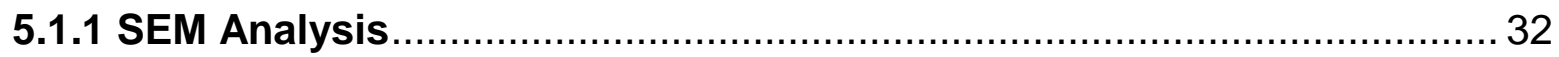

5.1.2 Size Distribution of Processed Powders.............................................. 35

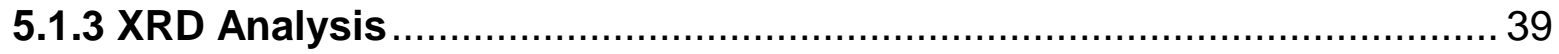

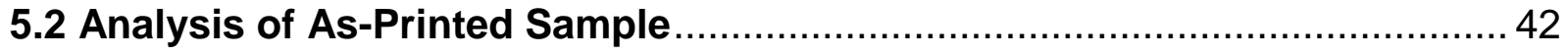

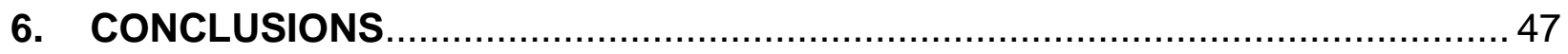

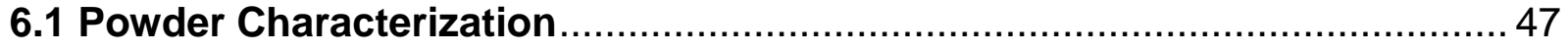

6.2 AM processing of MCB+BM powders .................................................. 47

7. RECOMMENDATIONS FOR FUTURE WORK .............................................. 48

A. Appendix

A.1 Mechanical Properties of powders/particles ..............................................

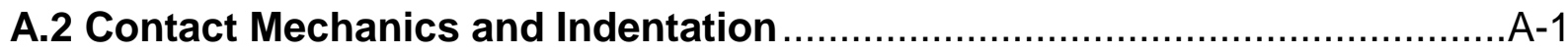

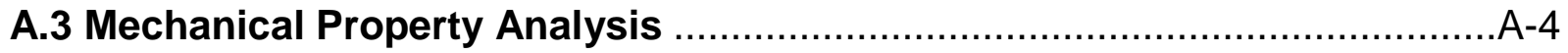

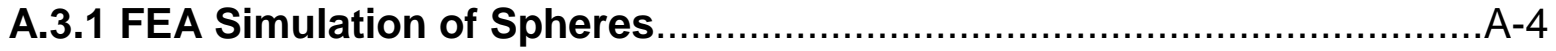

A.3.2 Experimental Multiple Loading-Partial Unloading Micro-indentation .A-11

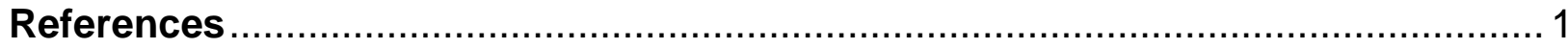




\section{LIST OF TABLES}

Table 2-1. Components of common Superalloys (wt. \%)[26] ............................... 11

Table 2-2. Components of common ODS alloys (wt. \%) [28] ............................. 12

Table 2-3. Assignment of factors and levels of $L_{16}\left(4^{5}\right)$ orthogonal array[34] ....... 19

Table 2-4. $L_{16}\left(4^{4}\right)$ orthogonal array matrix and response of grain size, D50 and

SSA[34]

Table 4-1.1 Breakdown of elemental powders used to produce ODS …................ 27

Table 4-2. Configuration of planetary ball milling parameters............................ 30

Table 5-1. The experimental density relative density, and input linear energy of as-

printed samples.

Table A-1. FEA Simulation Results of sphere with various sizes.

Table A-2. Experimental Results using Micro-Indentation Technique 


\section{LIST OF FIGURES}

Figure 1-1.Inconel 625 (a) average particle size of $160 \mu \mathrm{m}$ and size distribution of 60 $250 \mu \mathrm{m}$ and (b) average particle size of $100 \mu \mathrm{m}$ and size distribution of 45-120 $\mu \mathrm{m}[8] .2$ Figure 1-2. Images illustrate the gas and plasma Atomization process, respectively[14], [15]

Figure 1-3. SEM micrographs of Ti-6Al-4V powders produced by (a) gas atomization and (b) plasma atomization[13] ....

Figure 1-4. XRD data revealing dispersoid formation after heat treatment of powders under different chemical compositions[12] 3 Figure 1-5. TEM images of MCB processed ODS Ni-based powders (a) TEM BF image, (b) HRTEM image, (c) STEM EDX image [22]. 7

Figure 1-6. Types of strengthening mechanisms and corresponding effective working temperatures [23].

Figure 2-1. SEM images of $\mathrm{Y}^{\prime}$ strengthening phase in conventional Ni-based superalloys: (a). Wrought alloy and (b). Cast alloy [25] ...................................... 13 Figure 2-2. Initial, Middle and Final stages of the MA process technique. [18], [29] ..... 14 Figure 2-3. SEM images morphology of as-produced atomized powders (a) GA Ti6Al4V and (b) GA cross section IN718 [32] 16

Figure 2-4.Schematic of general powder bed system [35] .................................... 21

Figure 2-5. Schematic of general powder feed system [35] ...................................... 22

Figure 2-6. Schematic of general wire feed system [35].......................................... 22

Figure 4-1. SEM Micrographs of (a) Ni powder, (b) Cr powder, (c) Al powder, (d) W powder, (e) Y2O3 and (f) As-blended powders ...................................................... 28

Figure 4-2. HOSOKAWA NOBILTA-130 MCB Machine .......................................... 29 Figure 5-1. 300 rpm (a) BPR 15:1 15 hours (C1), (b) BPR 30:1 15 hours (C2), (c) 300 rpm BPR 30:1 45 hours (C3) ................................................................. 33 Figure 5-2. 400 rpm (a) BPR 15:1 15 hours (C4), (b) BPR 15:1 25 hours (C5), (c) BPR 15:1 30 hours (C6), (d) BPR 15:1 40 hours .................................................... 33 Figure 5-3. 300 rpm (a) BPR 15:1 15 hours (C1), (b) BPR 30:1 15 hours (C2), (c) 300 rpm BPR 30:1 45 hours (C3) ................................................................... 34 Figure 5-4. 400 rpm (a) BPR 15:1 15 hours (C4), (b) BPR 15:1 25 hours (C5), (c) BPR

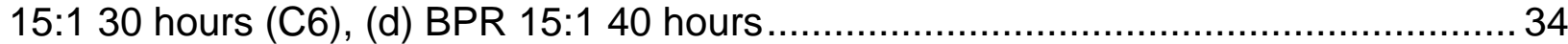
Figure 5-5. Variations of powder size distributions) [40] ....................................... 35 Figure 5-6. Powder size distribution of MCB+BM processed powder $400 \mathrm{rpm}$ BPR 15:1

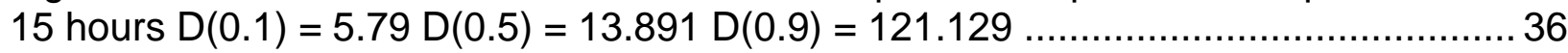
Figure 5-7. Powder size distribution of MCB+BM processed powder $400 \mathrm{rpm}$ BPR 15:1

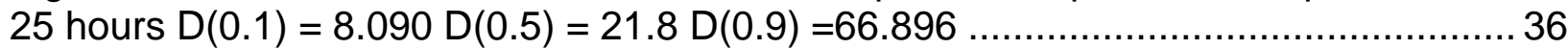
Figure 5-8. Powder size distribution of MCB+BM processed powder $400 \mathrm{rpm}$ BPR 15:1

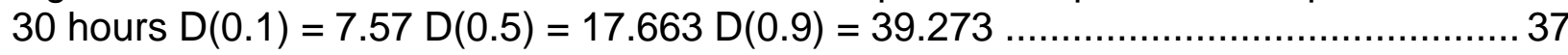
Figure 5-9. Powder size distribution of MCB+BM processed powder $400 \mathrm{rpm}$ BPR 15:1

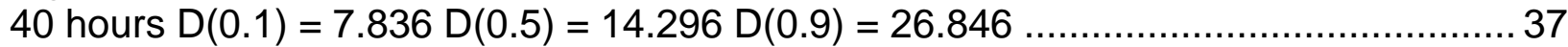
Figure 5-10.XRD patterns of pure elemental starting powders.................................. 39 Figure 5-11. XRD spectrum of MCB-only processed powders .................................. 40 
Figure 5-12.XRD spectrum of MCB+BM process at $300 \mathrm{rpm}$ (C1) BPR 15:1 and 15 hours (C2) BPR 30:1 and 15 hours (C3) BPR 30:1 and 45 hours.

Figure 5-13. XRD spectrum of MCB+BM process at BM milling speed of $400 \mathrm{rpm}$ and BPR of 15:1 (C4) 15 hours (C5) 25 hours (C6) 30 hours (C7) 40 hours.....

Figure 5-14. A schematic of the first batch of LENS as-printed samples[44]....

Figure 5-15. SEM showing images of the characteristic microstructures of as-printed ODS parts at different laser power and input linear energy: (a). 200W-6.06J/mm; (b). $250 \mathrm{~W}-7.75 \mathrm{~J} / \mathrm{mm}$; (c). $275 \mathrm{~W}-8.33 \mathrm{~J} / \mathrm{mm}$

Figure 5-16. High-magnification of SEM micrographs showing the characteristic microstructures of as-printed ODS parts in: (a). Figure 5-19 (a); (b). Figure 5-19 (b); (c). Figure 5-19 (c)...

Figure 5-17. EDS point analysis of as-printed ODS parts at different laser powder and input linear energy: (a). $250 \mathrm{~W}-7.75 \mathrm{~J} / \mathrm{mm}$ in mid-layer; (b). $275 \mathrm{~W}-8.33 \mathrm{~J} / \mathrm{mm}$ in mid-layer.

Figure A-1. Table-top load depth sensing indentation system (a) Schematic of indentation system and (b) indentation system [49].

Figure A-2. Experimental results from multiple loading partial unloading on $\mathrm{O} 1$ tool steel

(a) Experimental load-displacement curve and (b) linear relationship[49].

Figure A-3. Schematic of spherical specimen under the flat indenter with boundary

conditions shown.

A 5

Figure A-4. Mesh distribution of (a) analytical rigid indenter and (b) deformable sphereA6

Figure A-5. Stress Strain curve of Stainless Steel 316 at room temperature, $1400^{\circ} \mathrm{F}$, $1600^{\circ} \mathrm{F}$, and $1800^{\circ} \mathrm{F}[51]$

Figure A-6. FEA simulation of stiffness response of axisymmetric sphere $(D=10 \mathrm{~mm}) A-8$ Figure A-7. FEA simulation of stiffness response of axisymmetric sphere $(D=5 \mathrm{~mm})$..A-8 Figure A-8. FEA simulation of stiffness response of axisymmetric sphere $(D=3 \mathrm{~mm})$..A-9 Figure A-9. FEA simulation of stiffness response of axisymmetric sphere $(D=2 \mathrm{~mm})$..A-9 Figure $A-10$. FEA simulation of stiffness response of axisymmetric sphere $(D=1 \mathrm{~mm})$..A10

Figure $A-11$. FEA simulation of stiffness response of axisymmetric sphere $(D=0.5 \mathrm{~mm})$ 


\section{INTRODUCTION}

Combined with the capability of producing parts of complex geometries with efficient use of raw materials and minor waste, in recent years, metal additive manufacturing (AM) techniques have been under rapid development. This research will be focused on the development of special metal powders suitable for metal AM-assisted fabrication. Ni-based ODS alloys are favorable for gas turbine applications due to the combined capabilities of excellent mechanical strength at intermediate temperatures $\left(700-900^{\circ} \mathrm{C}\right)$ due to gamma-prime $\left(\mathrm{Y}^{\prime}\right)$ precipitation strengthening and oxide dispersion strengthening for creep resistance up to $1200^{\circ} \mathrm{C}$. Given the prospect of the rapid production of ODS alloys suitable for high temperature applications, the need to develop special ODS powder alloys is promising for the design, fabrication, and production of critical gas turbine components using metal AM technologies.

\subsection{Metal Powder Additive Manufacturing}

AM was first developed in the 1980's for creating models and prototypes layer by layer using computer-aided design (CAD). Thus, parts are built by bonding layers of material through high energy source, a binder or chemical instead of utilizing subtractive methods to remove materials from a larger piece[1]. AM techniques not only allow for rapid production of parts, unlike other methods, but also allow for complex geometries of parts. Powder Bed Fusion (PBF) and Directed Energy Deposition (DED) processes are common techniques for metal AM; PBF uses a high energy electron beam to melt and fuse material powder together, while DED utilizes a nozzle that deposits melted material on a build platform and the part rapidly solidifies [1,2]. The size, shape, and microstructure of the metal feedstock for PBF and DED processes influence key parameters of the end part, such as surface roughness and density of manufactured parts [3-5]. As a result, metal powders of particle size $10 \mu \mathrm{m}$ to $150 \mu \mathrm{m}$ and spherical in shape exhibit end parts with desirable mechanical properties, low porosity, and smooth surface finish. Typically, powders must be controlled in order to ensure repeatability for the production of end products; thus, many studies have been done to determine the optimized morphology and microstructure of powdered particles used for AM techniques.

For PBF, a near one-hundred percent relative density and smooth surface finish is ideal for the final product. As a result, particle size influences the minimum layer thickness and the minimal size of the part to be built[6], thus the desired layer thickness limits the maximum particle size for the powder. Also, if powders are too small, agglomeration of the fine powders will impede the efficiency of the high energy electron beam during the PBF process. With the agglomeration of fine powders on larger powders, the powders now have an irregular shape, unlike a spherical shape with optimized surface area; therefore, the produced part will have porosity. Although fine powders as used to produce end parts with low density and low surface roughness, the minimum particle size must be constrained to prevent agglomeration. 
On the other hand, parts produced using DED processes not only depend on the particle size and size distribution, but the high energy beam used to melt the powders. However, Kakinuma et al. shows fine powders with a narrow particle size distribution can lead to a uniform microstructure and high tensile strength[7]. The authors conducted a study on nickel-based superalloy Inconel 625, commonly used in aerospace applications, which require components with complex geometries. Inconel powder of average particle size $160 \mu \mathrm{m}$ (powder 1) and $100 \mu \mathrm{m}$ (powder 2), with size distribution of 60-250 $\mu \mathrm{m}$ and 45-120 $\mu \mathrm{m}$, respectively, was used for the DED process; Figure 1-1, below, shows the size, shape, and size distribution of the two powders used in the study. Conventional tensile tests were done on the DED AM produced parts and average tensile strengths in powders 1 and powders 2 were shown to be $738 \mathrm{MPa}$ and $778 \mathrm{MPa}$, respectively. The authors concluded, with analysis of the microstructure of the AM produced part, powder 2 showed a uniform microstructure due to the smaller average particle size and narrow size distribution when compared to powder $1[8]$.

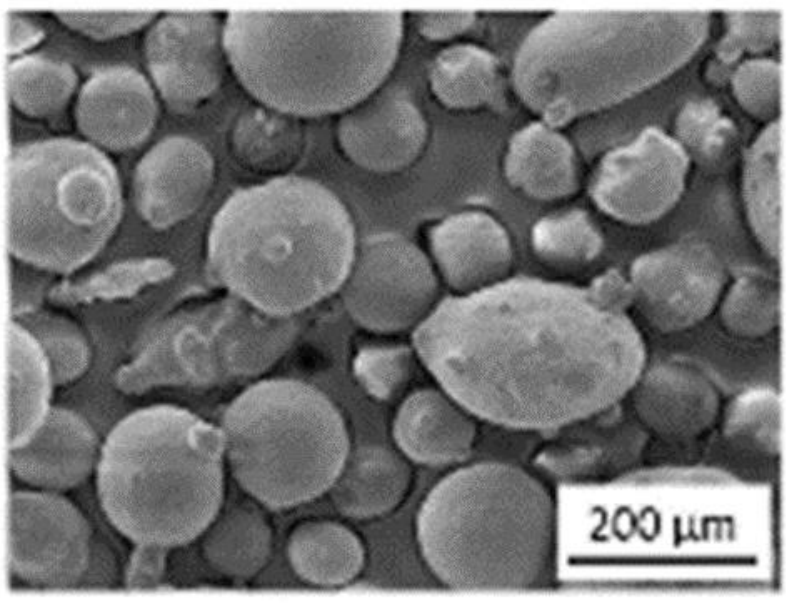

(a) Powder \#1

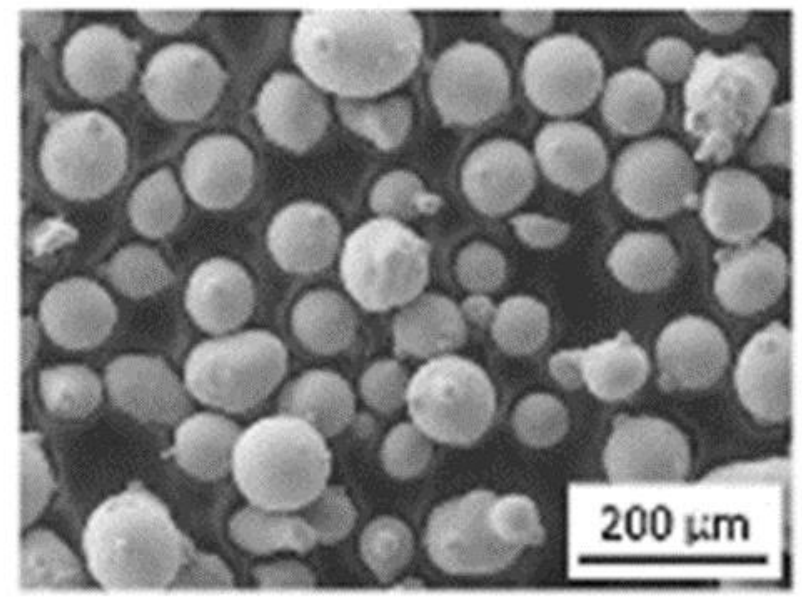

(b) Powder \#2

Figure 1-1.Inconel 625 (a) average particle size of $160 \mu \mathrm{m}$ and size distribution of 60$250 \mu \mathrm{m}$ and (b) average particle size of $100 \mu \mathrm{m}$ and size distribution of 45-120 $\mu \mathrm{m}[8]$.

Thus, for both PBF and DED processes, particle size, shape, and particle size distribution influence key factors of the end part. As of now, atomization processes are the leading metal powder fabrication techniques used to produce metal powders for AM processes.

\subsubsection{Atomization}

Atomization processes are capable of producing high quality metal powders, such as aluminum, brass, iron, stainless steel, and superalloys with uniform particle distribution and spherical shape. Gas atomization uses inert gas jets to atomize molten metal powders into fine droplets, which cool down as the droplets drop in the chamber and form spherical powders with uniform size distribution. On the other hand, plasma atomization uses DC arc plasmas to accelerate the inert gas breaking up the molten stream. A simple schematic of gas atomization and plasma atomization is shown in Figure 1-2 on the left 
and right, respectively. Both these process produce high quality metal powders with littleto-no contamination, uniform size distribution, and spherical shape [9-12], properties desirable for AM processes. The shape and size of Inconel 625, shown in Figure 1-2, and Ti-6Al-4V, shown in Figure 1-3, are alloyed powders produced by atomization. The Ti$6 \mathrm{Al}-4 \mathrm{~V}$ powders, produced by both gas and plasma atomization, has near spherical shape, $70 \mu \mathrm{m}$ average particle size, and particle size distribution from $50 \mu \mathrm{m}$ to $100 \mu \mathrm{m}$ [13]. However, given the cost and processing time of plasma atomization, gas atomization is the common technique for fabrication of metal alloy powders for AM production.
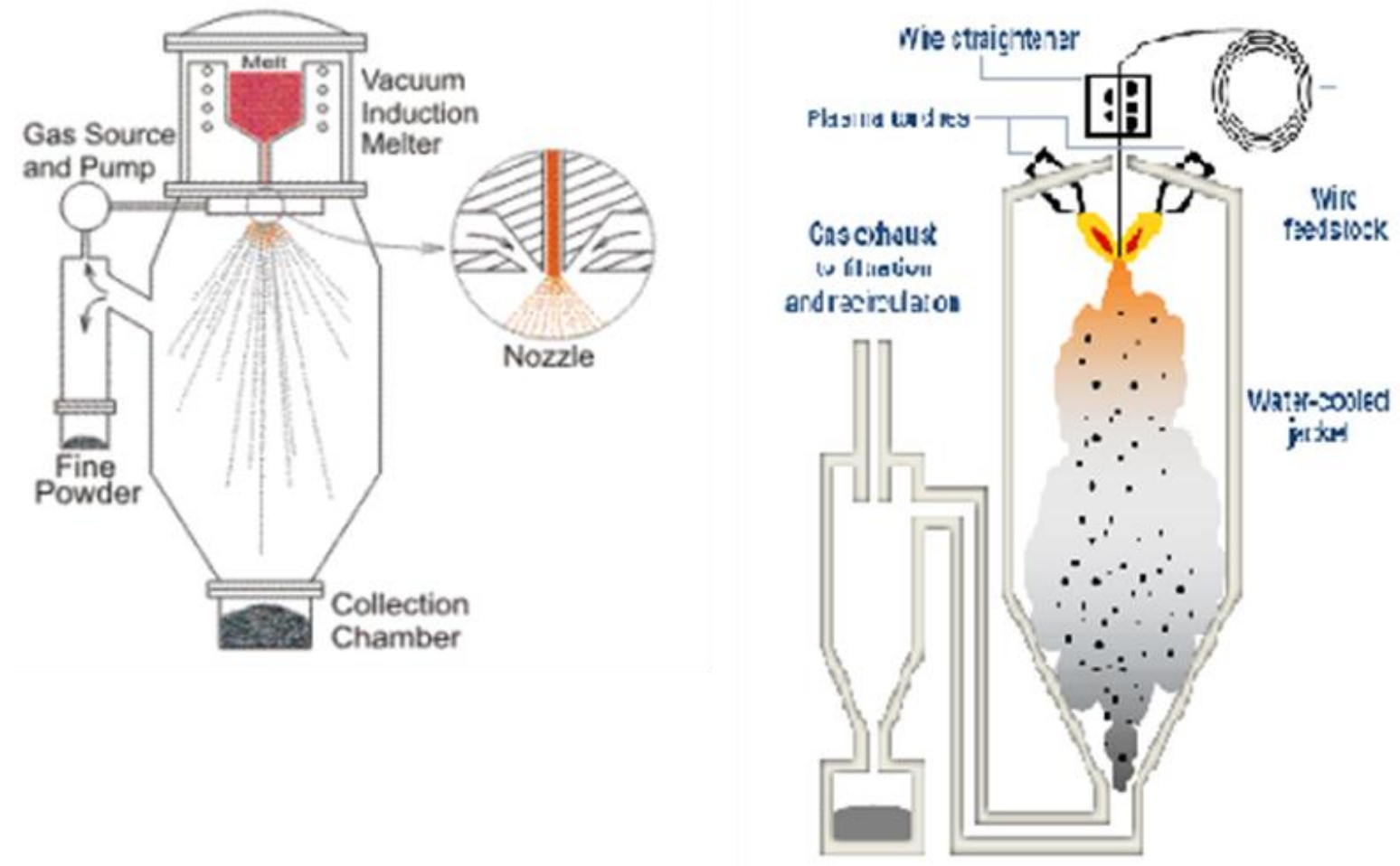

Figure 1-2. Images illustrate the gas and plasma Atomization process, respectively[14,15]
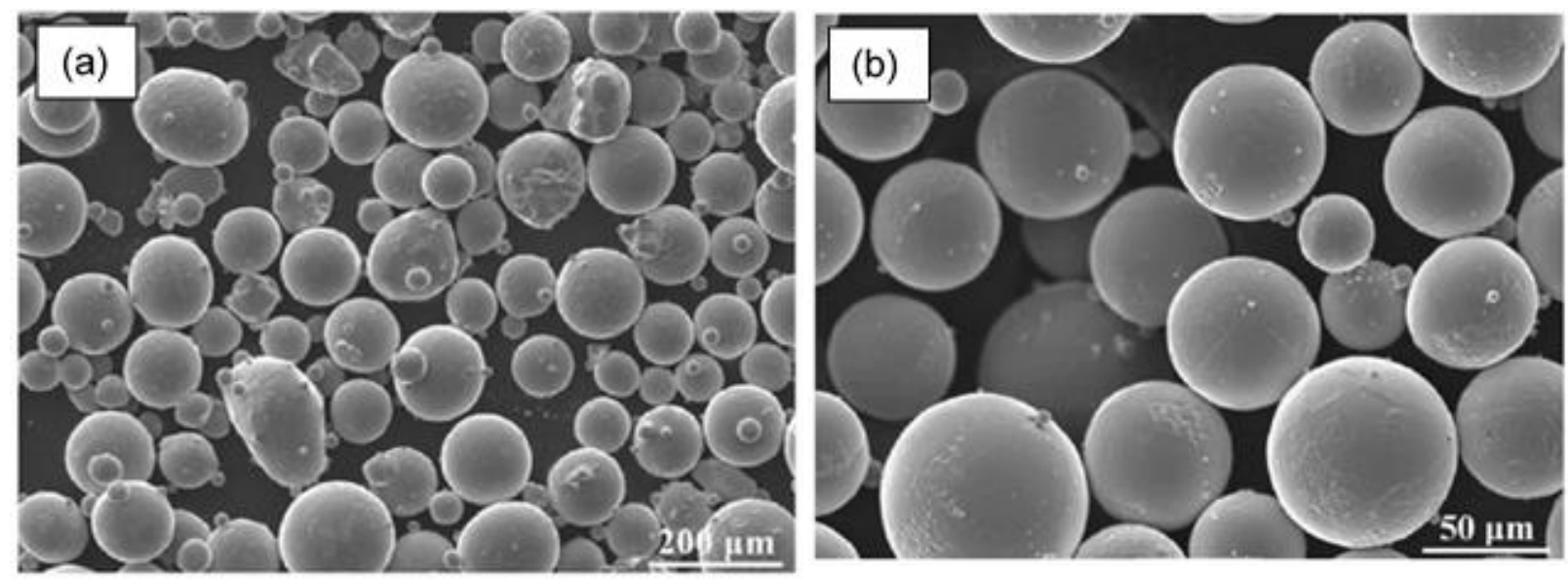

Figure 1-3. SEM micrographs of Ti-6Al-4V powders produced by (a) gas atomization and (b) plasma atomization[13] 


\subsection{Disadvantages of Gas Atomization}

Despite the capability of gas atomization process to produce powders with spherical shape, fine powder size, and narrow size distribution, iron or nickel-based Oxide Dispersion Strengthened (ODS) powders become problematic to produce because of the high melting temperature of yttrium (III) oxide $\left(\mathrm{Y}_{2} \mathrm{O}_{3}, 2425^{\circ} \mathrm{C}\right)$ compared to iron (Fe, 1538 $\left.{ }^{\circ} \mathrm{C}\right)$, nickel $\left(\mathrm{Ni}, 1668{ }^{\circ} \mathrm{C}\right)$, chromium $\left(\mathrm{Cr}, 1907{ }^{\circ} \mathrm{C}\right)$ and aluminum $\left(\mathrm{Al}, 660^{\circ} \mathrm{C}\right)$, thus, uniform dispersion of $\mathrm{Y}_{2} \mathrm{O}_{3}$ become problematic for production of ODS powders. However, post processing of the gas atomized powders or alterations to the conventional gas atomization process must be done in order to produce ODS alloyed powders. Conventionally, gas atomized powders are mechanically alloyed post process with $\mathrm{Y}_{2} \mathrm{O}_{3}$ in order to obtain uniform oxide dispersion and nanocrystalline microstructure. Rieken et al., also proposed a reactive gas atomization process for Fe-based ODS alloys which uses a reactive atomization gas to oxidize the surface of the ferritic alloys during the gas atomization process[12]. However, further processing, similar to utilizing mechanical alloying post gas atomization, was done to enable $\mathrm{O}$ exchange with $\mathrm{Y}$ precipitates to obtain uniform oxide dispersion. Figure 1-4 demonstrates the presence of the oxide dispersoid during all stages for the aforementioned reactive gas atomization process. It is evident from the XRD plot the as-atomized powder and the hot isostatic pressed (HIP) powder does not show oxide dispersoid formation until after heat treatment at $1200^{\circ} \mathrm{C}$. 


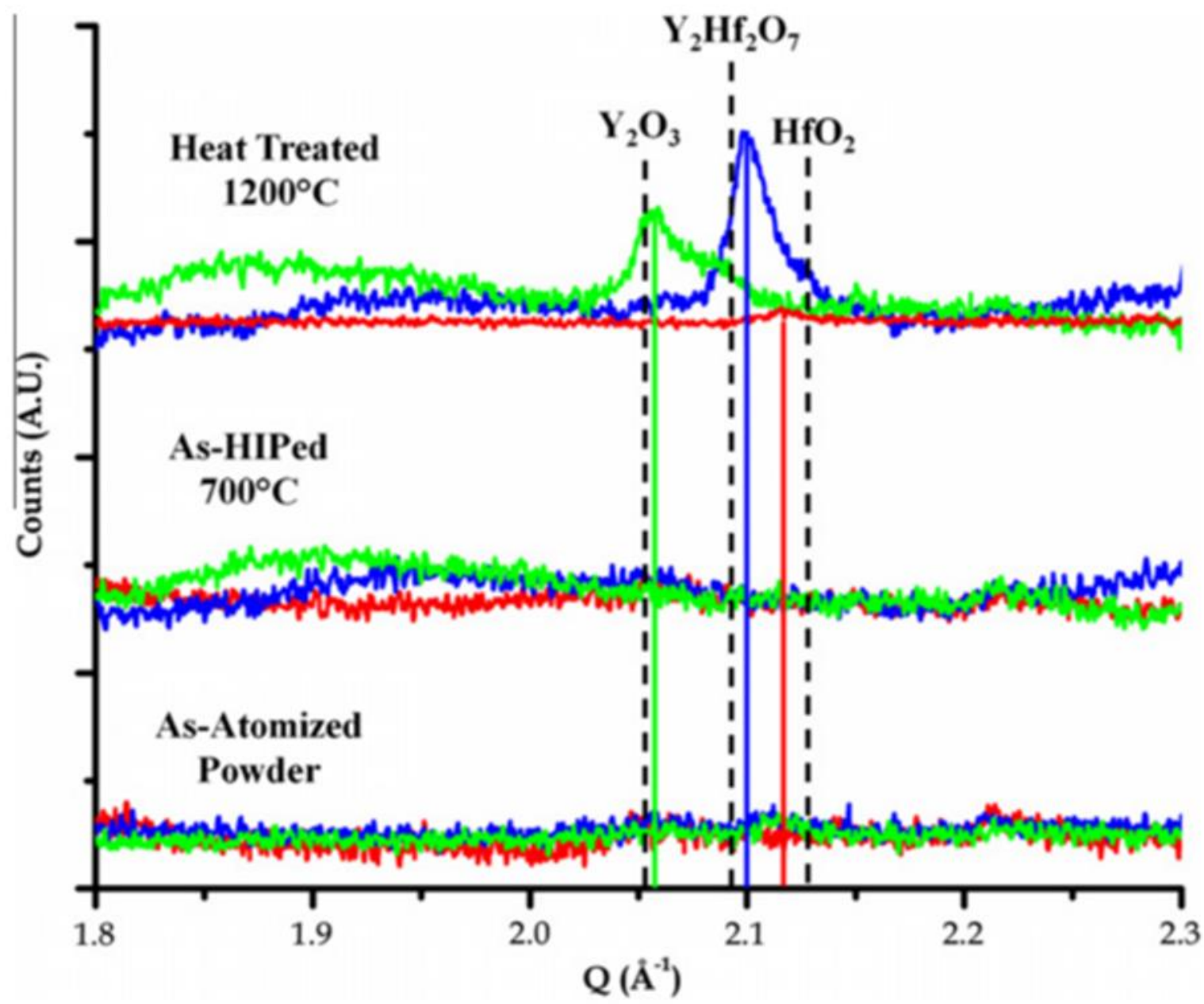

Figure 1-4. XRD data revealing dispersoid formation after heat treatment of powders under different chemical compositions[12]

Although this study shows evidence of the formation of oxide dispersoid in the gas atomized powders, proof of uniform distribution of the oxide is not clear. Moreover, formation of the dispersoids did not appear until further heat treatment of the atomized powders, which in turn will cause coarsening of the grain size. Mechanical alloying, however, excels in the production of ODS alloys with uniform oxide dispersion and nanocrystalline microstructure without further processing.

\subsection{ODS Production}

Nickel-based ODS alloys produced by MA process attract great attention as advanced high temperature materials [16-19]. ODS alloys retain useful strength up to high temperatures $\left(>1000{ }^{\circ} \mathrm{C}\right)$ due to strengthening of uniform dispersion of $\mathrm{Y}_{2} \mathrm{O}_{3}$ nanoparticles and intermediate temperatures $\left(700-900^{\circ} \mathrm{C}\right)$ due to gamma prime, $\mathrm{Y}^{\prime}$, precipitation hardening [20]. Mechanical alloying is a solid-state powder processing method that utilizes high energy ball milling or rod milling of elemental powder mixtures to produce homogenous alloyed particles with nanocrystalline microstructure[16],[18,19]. 
The starting elemental powders undergo cold-welding, fracturing, and rewelding continuously until the final composition of individual particles are of the same chemical composition (steady state conditions).

Typically, after the MA process, the alloyed powders are canned, degassed and consolidated by cold isostatic pressing (CIP), hot isostatic pressing (HIP) or hot extrusion followed by a final annealing and aging process to develop stable and recrystallized microstructure[21].

\subsection{MCB + BM}

Using a combination of the MCB technique and planetary BM, ODS Ni-based powders can be produced with nanocrystalline microstructure, uniform oxide dispersion, and $y^{\prime}$ precipitation. During the MCB process the starting powder mixtures are subjected to high compression, shear, and impact forces as they pass through a narrow gap in a high speed (4000-5000 rpm) rotating device. Earlier studies, utilizing MCB technique, showed uniform dispersion of $\mathrm{Y}_{2} \mathrm{O}_{3}$ with a thin layer of $20-25 \mathrm{~nm}$ on the master particles [22], as shown in Figure 1-5. 

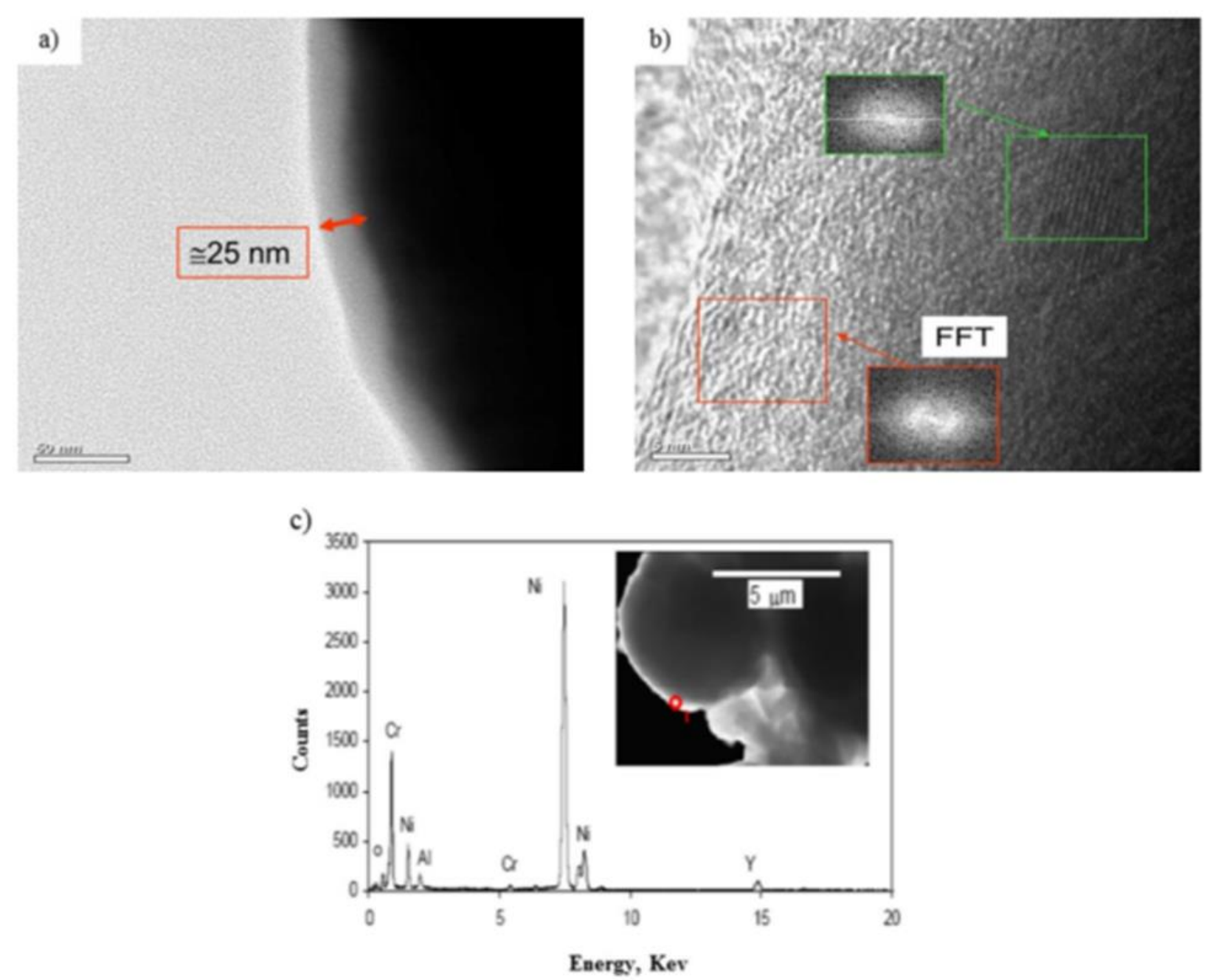

Figure 1-5. TEM images of MCB processed ODS Ni-based powders (a) TEM BF image, (b) HRTEM image, (c) STEM EDX image [22].

Figure 1-5 (a) shows the master particles $(\mathrm{Ni}, \mathrm{Cr})$ in darker contrast, surrounded by a thin film with lighter contrast; HRTEM analysis, shown in Figure 6(b), indicates the thin film to be an amorphous material and further analysis in STEM mode confirmed the presence of the constituent powders. As a result, the study determined the amorphous thin film dispersed and coated on the master particles are indeed the oxide dispersoid, $\mathrm{Y}_{2} \mathrm{O}_{3}$ [22]. Further processing of the MCB powders were done using planetary BM for different milling times, a ball-to-powder ration (BPR) of 30:1 and a speed of $300 \mathrm{rpm}$. The milling jars and milling media were of stainless steel and a range of sizes for the milling media was used to allow for efficient fracturing and cold welding of the MCB processed ODS powders. SEM EDX of the MCB plus BM processed powdered ODS showed the uniform distribution of the starting powders, or, in other words, the ODS particles had similar weight percentage to that of the starting chemical composition. Moreover, XRD analysis show a shift of the $\mathrm{Al}$ and $\mathrm{Ni}$ crystalline peaks for lower milling times and the disappearance of Al peaks and formation of NiAl with increased milling time. Furthermore, the XRD peaks broadened with increased milling time, which indicate a decrease in 
crystallite size of the ODS particles and potentially demonstrates the ability to produce ODS powder with nanocrystalline microstructure.

As shown, the combination of MCB and BM not only allow for uniform dispersion of $\mathrm{Y}_{2} \mathrm{O}_{3}$ unto the master particles, but also uniformly disperses $\mathrm{Al}$ and influences the formation of the NiAl matrix. This combination show potential of limiting the processing time of the ODS Ni-based powders when produced with BM only, while also producing particles with near spherical shape and narrow size distribution, given steady state milling operation is reached. However, adjustment of the process parameter of this combination can be done to improve the shape, size, size distribution and microstructure of the ODS powders.

\subsection{Motivation}

Given the capability of current AM techniques, such as PBF and DED, and the ability to fabricate Ni-based ODS powders with nanocrystalline microstructure, uniform oxide dispersion, and gamma prime precipitation with MCB plus BM, further parameter changes of the BM process allows increased opportunities for components under high temperature conditions. The capability of the AM process to not only create parts with complex geometries, but also, due to the rapid solidification/sintering of the metal alloy powders, can maintain the microstructure of the as-processed ODS powders.

\subsection{Objective}

The primary purpose of this study if to optimize the fabrication of Ni-based ODS powders suitable for AM application. Production of Ni-based ODS alloy particles spherical in shape, uniform size distribution, and average particle size larger than $10 \mu \mathrm{m}$ to be used for AM processes, primarily higher energy melting. The $\mathrm{y}^{\prime} \mathrm{Ni} 3 \mathrm{Al}$ intermetallic precipitate is the major strengthening phase of the Ni-based superalloy system. Unfortunately, at high working temperatures, the $\mathrm{Y}^{\prime}$ phase will dissolve and the superalloy loses the high temperature strength and creep resistance. The addition of $\mathrm{Y}_{2} \mathrm{O}_{3}$ improves the mechanical properties at high temperatures because the nanoparticle is considered a secondary particle in superalloys. Thus, $\mathrm{Y}_{2} \mathrm{O}_{3}$ is capable of pinning the dislocation and grain boundaries of the ODS microstructure.

Figure 1-6 shows the types of strengthening mechanisms and effective working temperatures of superalloy systems. Ni-based ODS alloys are considered in the dispersion strengthening category; the melting point of Ni alloys range between 1400$1500^{\circ} \mathrm{C}$, thus, the effective working temperature of Ni-based ODS alloys can fall in the range of $1250-1350^{\circ} \mathrm{C}$. As a result, the effective working temperature of this alloy system is in the range of the exhaust temperature for turbines. Although gas atomization is the most used technique for superalloy fabrication, the process is incapable of producing ODS alloys. The highest know melting temperature during the gas atomization process is $1700^{\circ} \mathrm{C}$ and melting temperature of oxides in known ODS alloys can be larger than $2000^{\circ} \mathrm{C}$. 


\begin{tabular}{|c|c|c|}
\hline $\begin{array}{c}\text { Strengthening } \\
\text { Mechanism }\end{array}$ & $\begin{array}{c}\text { Effective } \\
\text { Temperature }\end{array}$ & Comments \\
\hline Work Hardening & $\sim 0.3 \mathrm{~T}_{\mathrm{m}}$ & Dislocation/Dislocation Interaction \\
\hline $\begin{array}{c}\text { Grain Size } \\
\text { Refinement }\end{array}$ & $\sim 0.3 \mathrm{~T}_{\mathrm{m}}$ & $\begin{array}{c}\text { Grain Boundary Area - Dislocation } \\
\text { Interaction }\end{array}$ \\
\hline $\begin{array}{c}\text { Solid Solution } \\
\text { Strengthening }\end{array}$ & $\sim 0.4 \mathrm{~T}_{\mathrm{m}}$ & $\begin{array}{c}\text { Lattice Strain Fields: Modulus Change, } \\
\text { dislocation interaction }\end{array}$ \\
\hline $\begin{array}{c}\text { Precipitation } \\
\text { Strengthening }\end{array}$ & $\sim 0.6 \mathrm{~T}_{\mathrm{m}}$ & $\begin{array}{c}\text { Metastable } 2^{\text {nd }} \text { Phase Particles, impede } \\
\text { dislocation motion }\end{array}$ \\
\hline $\begin{array}{c}\text { Dispersion } \\
\text { Strengthening }\end{array}$ & $\sim 0.9 \mathrm{~T}_{\mathrm{m}}$ & $\begin{array}{c}\text { Insoluble } 2^{\text {nd }} \text { Phase, stabilize grain, } \\
\text { subgrains and creep }\end{array}$ \\
\hline
\end{tabular}

Figure 1-6. Types of strengthening mechanisms and corresponding effective working temperatures [23].

Since the early 1970's the MA process has been the most widely used fabrication technique of ODS alloys. The MA process is capable of mixing, cold-welding, fracturing, and rewelding raw elemental powders in a high energy mill. The oxide nanoparticles are uniformly dispersed onto the master particles during this process. Until recently, the finished ODS powders are subjected to Hot Isostatic Pressing (HIP) to consolidate the powders and heat treatment is used to rid of residual stresses. The HIPed powders are then hot worked for further consolidation and undergoes more heat treatment. Thus, the conventional various post processes taken to fabricate ODS alloys can be expensive and time consuming.

As a result, a novel MCB+BM process will the utilized to produce Ni-based ODS powders and parameter manipulation of the BM process will be explored. The MCB technique is a dry powder process that can form alloys through solid state diffusion. Kang et al. has shown the MCB process can uniformly disperse $\mathrm{Y}_{2} \mathrm{O}_{3}$ nanoparticles onto the host $\mathrm{Ni}, \mathrm{Cr}$ particles to form composite powders [22]. It has also been shown the BM technique is capable of producing powders with nanocrystalline microstructure and evidence of the intermetallic $y^{\prime}$ precipitates [24]. As a result, the combination of the $\mathrm{MCB}+\mathrm{BM}$ process is an improvement and alternative to the conventional ODS alloy fabrication currently available. Given the $M C B+B M$ processed powders contain a nanocrystalline microstructure, investigation of the individual powders is also of importance.

Additive Manufacturing will also be used to produce bulk and densified samples of the Ni-based ODS powders. Preliminary investigation of the mechanical property of individual powders can lead to a customization of the AM process for development. The AM technique is capable of achieving bulk samples in a simpler manner compared to the conventional powder consolidation technique used for ODS alloys. However, there are some limitations and challenges to this work. The primary challenge of this research is parameter selection of the BM process to produce ODS powders suitable for AM techniques. The powder morphology, average size, size distribution, and microstructure plays a crucial role in the operating parameters and feasibility of AM use. As a result, this 
research will focus on the production of $\mathrm{Ni}$-based ODS powders given the manipulation an adjustment of the BM process parameters. 


\section{LITERATURE REVIEW}

\subsection{Superalloys and Oxide Dispersion Strengthened (ODS) Superalloys}

Superalloys, commonly used in high temperature turbine applications, refer to iron, nickel, and cobalt based alloys. Superalloys are useful because of good high temperature strength, high ductility at high temperatures, excellent creep strength, and good oxidation/corrosion resistance. Table 2-1 shows the composition of conventional superalloys, including the popular Iron-Nickel based superalloy Inconel 718 (IN718) [25].

Table 2-1. Components of common Superalloys (wt. \%)[26]

\begin{tabular}{ccccccccc} 
Alloy & $\mathrm{Ni}$ & $\mathrm{Fe}$ & $\mathrm{Cr}$ & $\mathrm{Ti}$ & $\mathrm{Al}$ & $\mathrm{Mo}$ & $\mathrm{C}$ & Other \\
\hline IN 100 & $\mathrm{Bal}$ & - & 12.4 & 4.3 & 5 & 3.2 & $0 . .07$ & $18.5 \mathrm{Co}$ \\
Rene 88 & $\mathrm{Bal}$ & - & 16 & 3.7 & 2.1 & 4 & 0.03 & $13 \mathrm{Co} 4 \mathrm{~W} 0.7 \mathrm{Nb}$ \\
IN 718 & $\mathbf{5 0}$ & $\mathbf{3 0}$ & $\mathbf{1 8}$ & $\mathbf{0 . 8}$ & $\mathbf{0 . 6}$ & $\mathbf{3}$ & $\mathbf{0 . 0 5}$ & $\mathbf{5 . 2 N b}$ \\
Waspaloy & 58 & - & 19 & 3 & 1.4 & 4.4 & 0.08 & $13 \mathrm{Co}$ \\
Rene 80 & $\mathrm{Bal}$ & - & 14 & $\mathbf{5}$ & $\mathbf{3}$ & 4 & 0.17 & $9.5 \mathrm{Co}$ W
\end{tabular}

Unfortunately, current superalloys cannot meet the requirements for industrial applications. With an increase in time, the strength of the superalloy is weakened when the working temperature is $50 \%$ of the melting point of said alloy. As a result, addition of oxide dispersoids within the superalloy alloys for enhanced mechanical strength at high temperatures. ODS alloys are the common type of dispersion strengthened superalloys which a new element, harder than the base metal of the alloy, is introduced as a secondary phase particle. Notable strengthening particles are refractory Oxides (i.e., $\mathrm{Y}_{2} \mathrm{O}_{3}, \mathrm{Al}_{2} \mathrm{O}_{3}, \mathrm{HfO}_{2}$, and $\mathrm{ThO}_{2}$ ), Carbides (i.e., TiC, $\mathrm{SiC}$, and $\mathrm{WC}$ ), Nitrides (i.e., TiN, BN, and $\mathrm{Si}_{3} \mathrm{~N}_{4}$ ), and Borides (i.e., $\mathrm{TiB}_{2}, \mathrm{ZrB}_{2}$, and $\mathrm{CrB}_{2}$ ). The addition of dispersoid strengthening results in the dispersion strengthening $Y^{\prime}$ phase, distinguishing ODS alloys from conventional superalloys.

Given the advantage of ODS alloys, the material characteristic of the oxide dispersoid is critical. Dispersoids with high melting temperatures, chemical stability, insolubility in the solid solution, and low diffusion rate are necessary for ODS alloys. In early 1970's, thorium dioxide $\left(\mathrm{ThO}_{2}\right)$ was the common oxide dispersoid used for ODS alloys until replaced by yttrium (III) oxide $\left(\mathrm{Y}_{2} \mathrm{O}_{3}\right)$ due to its radioactivity. $\mathrm{Y}_{2} \mathrm{O}_{3}$ also can form more stable metal composite oxides due to excess oxygen and $\mathrm{Al}$ in the ODS alloy matrix. Srinivasan et al. provide significant evidence dispersion of $\mathrm{Y}_{2} \mathrm{O}_{3}$ consist of several mixed oxides, i.e., $\mathrm{Y}_{3} \mathrm{Al}_{5} \mathrm{O}_{2}$ (YAG), $\mathrm{YAIO}_{3}$ (YAP), $\mathrm{YAH}$, etc. [27]. Table 2-2 demonstrates the commonly used ODS alloys and the corresponding composition given the compound $\mathrm{Y}_{2} \mathrm{O}_{3}$ as the oxide dispersoid to inhibit dispersoid strengthening. ODS Ni-based superalloy and ODS Fe-based superalloy are the most studied. 
Table 2-2. Components of common ODS alloys (wt. \%) [28]

\begin{tabular}{ccccccccc} 
Alloy & $\mathrm{Ni}$ & $\mathrm{Fe}$ & $\mathrm{Cr}$ & $\mathrm{Ti}$ & $\mathrm{Al}$ & $\mathrm{C}$ & $\mathrm{Y}_{2} \mathrm{O}_{3}$ & Other \\
\hline MA 754 & $\mathrm{Bal}$ & 1.0 & 20 & 0.5 & 0.3 & 0.05 & 0.6 & \\
MA 757 & $\mathrm{Bal}$ & - & 16 & - & 4.5 & - & 1 & \\
MA 758 & $\mathrm{Bal}$ & - & 20 & 0.5 & 0.3 & - & 0.6 & \\
PM 1000 & $\mathrm{Bal}$ & - & 20 & - & 0.3 & - & 0.6 & $0.5 \mathrm{Mo}$ \\
MA 6000 & Bal & - & 15 & 2.5 & 4.5 & 0.05 & 1.1 & 2 2Mo4W2Ta \\
MA 956 & - & Bal & 20 & 1 & 4.5 & 0.02 & 0.5 & \\
PM 2000 & - & Bal & 20 & 0.5 & 5.5 & $<0.04$ & 0.5 &
\end{tabular}

\subsubsection{ODS Ni-based Superalloys}

The first ODS Ni-based alloy was produced in the mid-1900's by the DuPont company by a co-precipitation method to generate conventional Td-Ni ODS alloys. Eventually, in the early 1970's, John Benjamin and his team developed the mechanical alloying (MA) technique in order to produce ODS alloys. The selection of the oxide dispersoid is crucial for the production of Ni-based superalloys. The chemical stability, dissolution in superalloy matrix, diffusion coefficient, and interphase energy are key characteristics that determine the choice of oxide particle. The strength of most alloys decreases with increasing temperature, with the exception of few intermetallic compounds. $\mathrm{Y}^{\prime}-\mathrm{Ni}_{3}(\mathrm{Al}, \mathrm{Ti})$ is considered primary strengthener of Ni-based superalloys. The precipitates, however are metastable and will coarsen when heated past temperatures of $900^{\circ} \mathrm{C}$. Introducing stable nanoparticles such as $\mathrm{Y}_{2} \mathrm{O}_{3}, \mathrm{ThO}_{2}$, and $\mathrm{ZrO}_{2}$ can disperse homogeneously through the ODS alloy matrix, increasing the strength of the material at higher temperatures [26].

ODS Ni-based superalloys can be classified as alloys with low Al and Ti content or alloys with high $\mathrm{Al}$ and Ti contents. ODS Ni-based superalloys with low $\mathrm{Al}$ and $\mathrm{Ti}$ content does not include the strengthening $Y$ ' phase. As a result, high temperature strengthening depends on the solid solution strengthening of $\mathrm{Cr}$ : The combination of $\mathrm{Cr}$ and $\mathrm{Al}$ improves oxidation resistance in the alloys and average medium temperature strength. On the other hand, Ni-based ODS alloys with higher $\mathrm{Al}$ and $\mathrm{Ti}$ concentration shows more $\mathrm{y}^{\prime}$ strengthening in the Ni matrix. The latter also can consist of Mo, W or Ta which contributes to stabilizing the strengthening phase and leading to higher strength at medium temperatures. Extrusion, rolling, and hot compression are used to obtain desirable columnar grain structures for improved endurance strength and creep resistance. Figure 2-1 shows the $Y$ ' phase of conventional ODS Ni-based superalloys. The strengthening phase is seen as spheroidal and cuboidal with some degree of coherence [25]. 

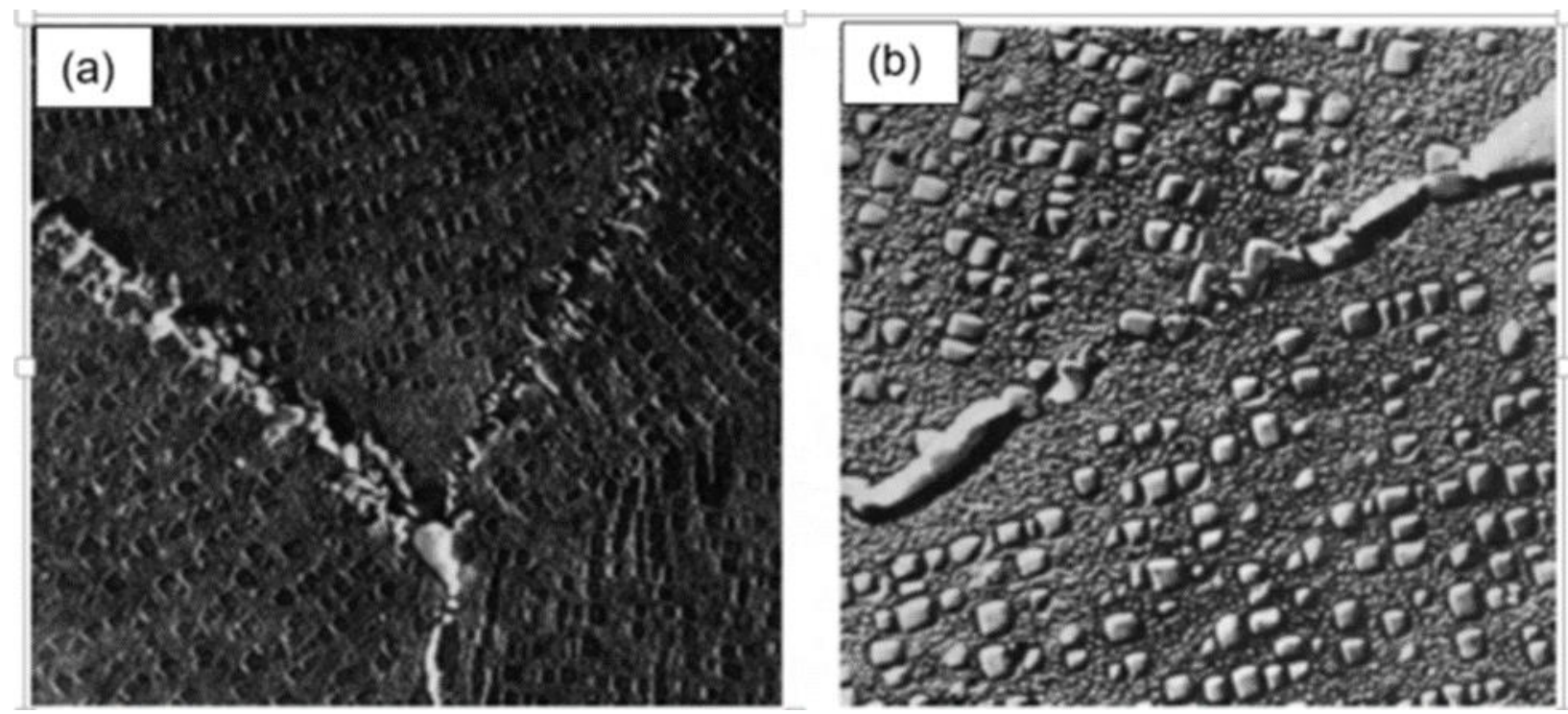

Figure 2-1. SEM images of $\gamma^{\prime}$ strengthening phase in conventional Ni-based superalloys: (a). Wrought alloy and (b). Cast alloy [25]

\subsection{Fabrication Techniques of ODS alloy Powders}

\subsubsection{Mechanical Alloying (MA)}

The MA process uses a high energy ball milling machine to combine two or more powders to form fine-grained homogeneous alloyed powders. The process involves the repeated cold welding, fracturing, and rewelding of powders; the MA process can be broken down into ductile-ductile, brittle-brittle, and ductile-brittle interactions. Ni-based ODS alloys will be classified as a ductile-brittle interaction which the oxide dispersoid $\left(\mathrm{Y}_{2} \mathrm{O}_{3}\right)$ are brittle powders and the master powders (Ni, $\left.\mathrm{Cr}\right)$ are ductile. The process can be simplified into three stages, demonstrated in Figure 2-2 below. 

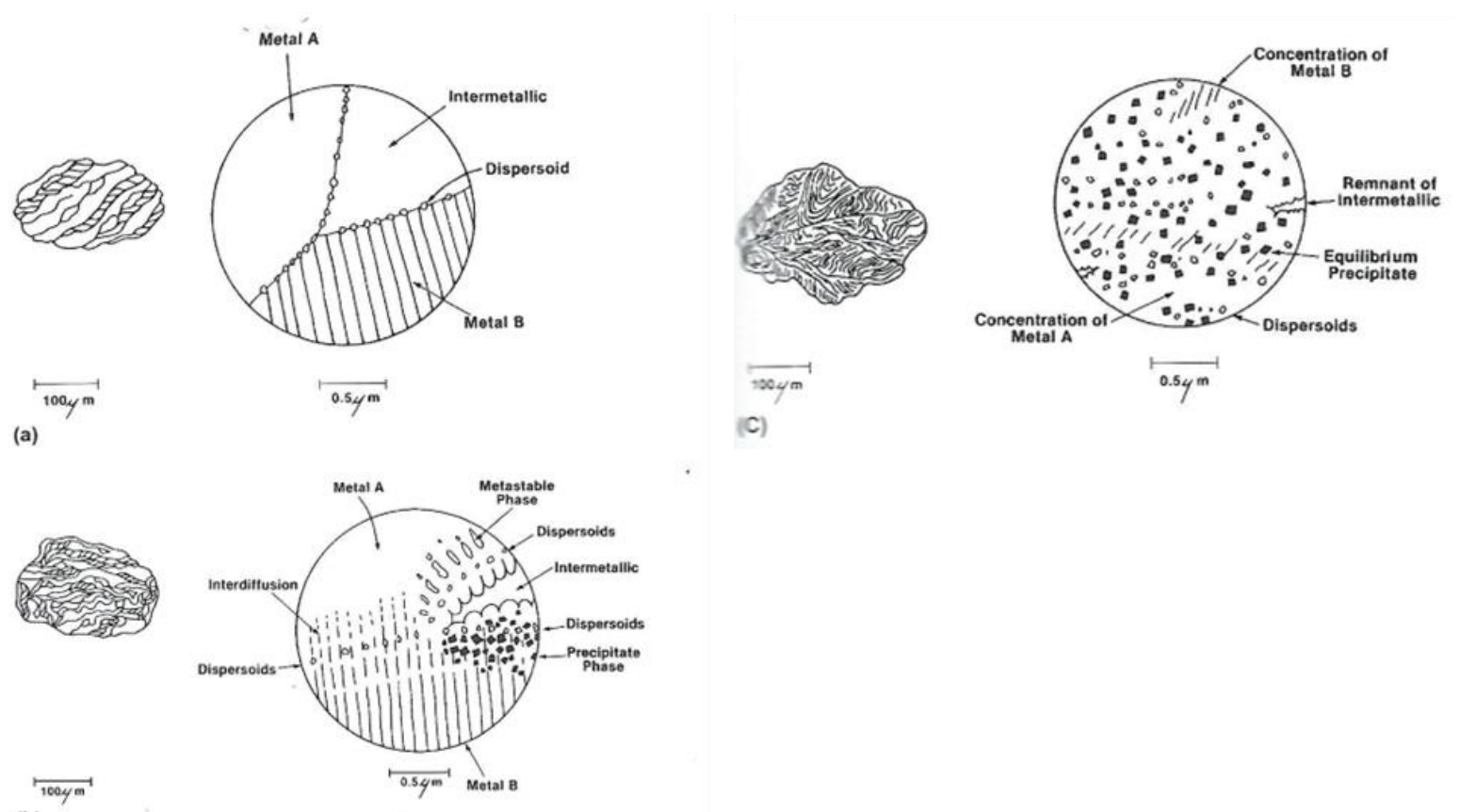

(b)

Figure 2-2. Initial, Middle and Final stages of the MA process technique. [18,29]

Figure 2-2 (a) shows MA process of Metal A, Metal B, and an oxide dispersoid in initial stages of the process. The ductile master particles are flattened, creating lamellar powders, by collision and the brittle oxides are fragmented. The fragmented particles tend to trap in the ductile particles and appear closely spaced along the interlamellar spacing. As further milling takes place, the second stage of the alloying process is seen (Figure 22 (b)). Due to the rewelding during the MA process, the ductile powders are work hardened and the lamellae is refined. With the lamellar refinement, the lamellae become convoluted with the brittle oxide dispersoid constituents. Thus, the composition of the individual particles begins to converge towards the initial composition of the starting powders. Figure 2-2 (c) demonstrates the final stages of the MA process; there is further refinement of the lamellae, decrease interlamellar spacing, and the insoluble oxide dispersoids are uniformly dispersed within the lamellae [18]. The final stage, considered steady state of the MA process, all individual powders have the same composition of the starting blend, uniform dispersion of the given oxide dispersoid, and nanocrystalline microstructure $[18,30,31]$.

Most MA techniques use medium to deliver the high energy impact needed for fracturing and cold welding. Steel balls of varying sizes or similar size is common for fabrication of Ni-based ODS powders. The containers are the same composition of the medium to prevent contamination and a ball-to-powder ratio (BPR) of 1:10 up to 1:40 is used depending on the powder, medium, and previous studies. Thus, the MA process is dominated by solid diffusion. High energy impact causes high-density crystal defects during the process, thus allows for metal atoms diffuse and grow along the free surface of crystals, grain boundaries and lattice until the constituents form an alloy [18]. However, 
MA techniques with use of a medium requires anywhere between 20-100 hours of operation.

\subsubsection{Mechano-Chemical Bonding (MCB)}

Unlike common MA techniques, MCB process does not use medium to fracture and cold weld the starting blend of the constituent powder. The MCB technique passes dry powders through a narrow gap, allowing smaller particles to bond and coat the surface of the large master powders [22]. Only using the internal mechanical energy without any binders or medium, a solid solution may form which small particles diffuse into the host particles. The process allows for uniform dispersion of oxide particles in ODS alloy with little to no contamination from outside sources, i.e., medium for grinding and process control agents (PCA).

The MCB technique is capable of producing Ni-based and Fe-based ODS alloy powders. The major component of the MCB machine are the rotors and blades with press heads. The rotor and blades rotate at speeds as high as $6000 \mathrm{rpm}$, promoting collision and high energy impact of individual particles without use of medium. Earlier studies, utilizing $\mathrm{MCB}$ technique, showed uniform dispersion of $\mathrm{Y}_{2} \mathrm{O}_{3}$ with a thin layer of 20-25 nm on the master particles. TEM studies indicate the thin film to be an amorphous material and further analysis in STEM mode confirmed the presence of the constituent powders. As a result, the study determined the amorphous thin film dispersed and coated on the master particles are indeed the oxide dispersoid, $\mathrm{Y}_{2} \mathrm{O}_{3}$ [22]. MCB process allows for short operation time, uniform dispersion of $\mathrm{Y}_{2} \mathrm{O}_{3}$, and alloy $\mathrm{Y}$-NiAl matrix.

\subsection{Atomization}

Atomization process is the conventional technique used to fabricate superalloys especially used for AM processing. The process manipulates the low mechanical strength of liquid metal to break into metal droplets. High pressure inert gas or solution is sprayed at high speeds unto the metal droplets for solidification with high cooling rate. Atomization process can be used to produce high quality $\mathrm{Ni}$, Fe, and $\mathrm{Al}$ superalloys with homogenous composition and spherical shape. The process is broken into two steps: shearing of the liquid and freezing of the liquid droplets. The shearing of the liquid metal can be supplied from several sources, the common sources are gas atomization (GA) and water atomization (WA).

\subsubsection{Gas Atomization (GA)}

Gas atomization uses inert gas jets to atomize molten metal powders into fine droplets, which cool down as the droplets drop in the chamber and form spherical powders with uniform size distribution. During the GA process, the inert gas jet atomize the molten metal, forms fine metal droplets, and cools the metal droplets as the fall in the atomizing column thus leading to formation of pre-alloyed powders. GA is common in metal powder metallurgy due to ease of use, processing time, and cost. However, the highest known melting temperature of technique can achieve is $1700^{\circ} \mathrm{C}$, lower than the 
melting temperatures of majority of oxides. As a result, fabrication of ODS alloys will be troublesome for oxides such as $\mathrm{Y}_{2} \mathrm{O}_{3}$, which has a melting temperature of $2425^{\circ} \mathrm{C}$. The oxide will not be melted for uniform dispersion in the molten metal pool, causing complications in the process.

Rieken et al., proposed a reactive gas atomization process for Fe-based ODS alloys which uses a reactive atomization gas to oxidize the surface of the ferritic alloys during the atomization process[12]. However, further processing, similar to utilizing mechanical alloying post gas atomization, was done to enable $O$ exchange with $Y$ precipitates to obtain uniform oxide dispersion. Figure 4 demonstrates the presence of the oxide dispersoid during all stages for the aforementioned reactive gas atomization process. It is evident from the XRD plot the as-atomized powder and the hot isostatic pressed (HIP) powder does not show oxide dispersoid formation until after heat treatment at $1200^{\circ} \mathrm{C}$. Figure 2-3 shows SEM images of morphology and size distribution of asproduced gas atomized powders.
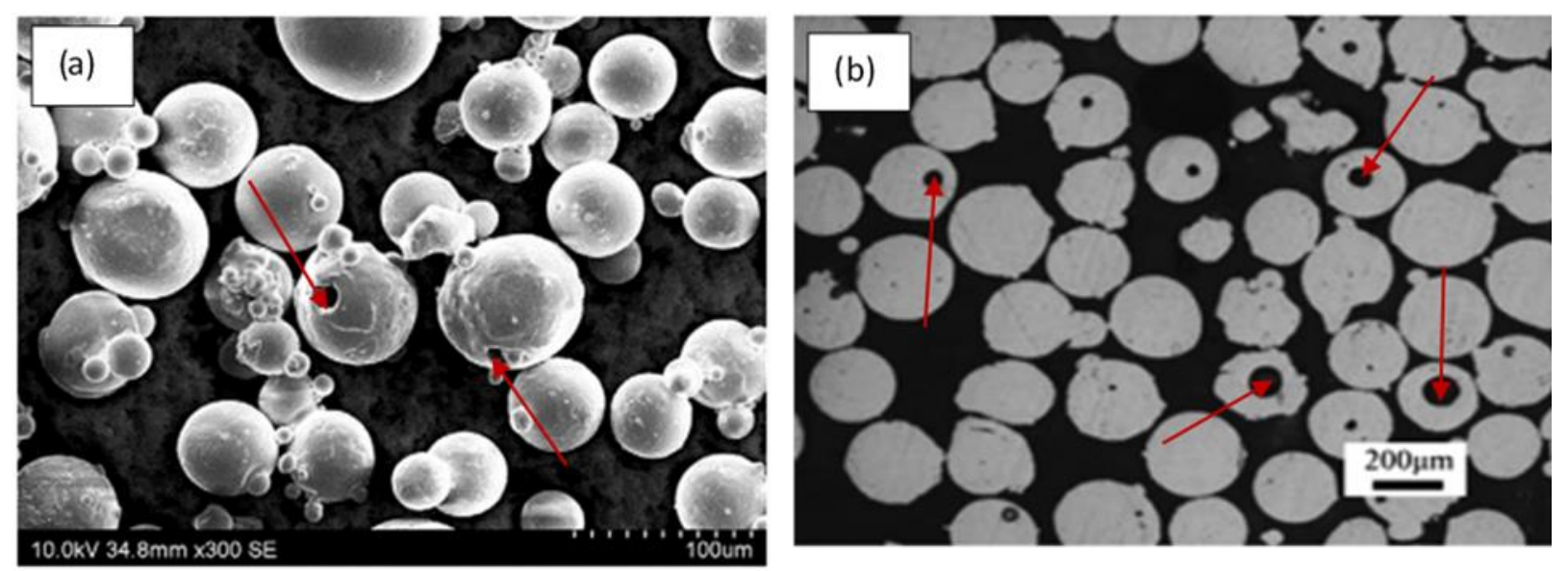

Figure 2-3. SEM images morphology of as-produced atomized powders (a) GA Ti6Al4V and (b) GA cross section IN718 [32]

The SEM images show spherical superalloy powders with little agglomeration and uniform size distribution. On the other hand, as indicated by the arrows in the images, pores caused during the solidification of the GA process can occur. The pores inside the particles would affect the density and microstructure of AM printed samples [33]. It is clear the GA process is capable of fabricating superalloy powders with uniform size distribution and spherical shape suitable for AM applications, however further processing to create ODS alloy and development of pores during solidification does not make GA an ideal process.

\subsubsection{Water Atomization (WA)}

Unlike GA process, water atomization process atomizes the molten metal pool with water jets. The metal stream is blown out in a fine jet while water from a highpressure pump blows onto the metal. The resulting powders are further dried and sieved for powder size classification. The resultant ODS particles are found to be not 
perfectly spherically shaped or irregular in shape. Also, the use of a liquid solution for atomization and solidification can cause contamination of the superalloy powders [10]. WA process has the same limitation of GA process, causing further processing after atomization in order to obtain ODS alloy.

\subsection{Mechanical Alloying via Ball Milling}

The MA process starts with mixing of powders with suitable chemical composition and loading powder mix with grinding medium. The mixture is milled for a set time until the individual powders reach a steady state. Each particle is not only alloyed, but have the same composition of the starting powder mix. The MA process is affected by the raw materials, milling type and processing variables.

\subsubsection{Parameters of Planetary Ball Milling}

The following process variables are critical to achieve product phase, microstructure, morphology, and size distribution of ODS alloys produced via planetary BM:

- Milling container

- Milling energy/speed

- Milling time

- Type size and size distribution of grinding medium

- Ball-to-powder ratio (BPR)

- Extent of vial filling

- Milling atmosphere

- Process Control Agent (PCA)

The type of milling container is important due to impact $f$ the grinding medium on the inner walls of the container. As a result, material from the container can dislodge into the powder and contaminate the material. Work hardened steel, stainless steel, tungsten carbide coated steel, and yttria-stabilized zirconia (YSZ) are specific materials used for milling containers depending on starting constituent powders. Stainless steel and tungsten carbide coated steel are most common milling jars used for production of ODS alloys [29].

The milling speed during the MA process a key role in energy input to the starting powders. Higher speed results in more impact/higher energy to the starting powders, however, at critical speeds, the medium can be pinned to the inner walls of the milling container. In other words, higher speed is necessary for the MA process and the optimal speed should be slightly below the critical speed. Higher speeds during MA process may also lead to higher temperature in milling containers. The increased temperature can be advantageous and disadvantageous for the starting powders. High temperature can promote diffusion of the oxide dispersoid in the lamellae; on the other hand, increase temperature can decompose supersaturated solid solutions or metastable stables formed during the MA process [30]. 
The most important parameter in MA process is the milling time. The milling time is determined when a steady state is reached between the fracturing and cold welding of the starting powders. The milling time depends on the milling type, milling energy, BPR, and temperature. Lastly, the powders to be mechanically alloyed plays the largest role in determination of milling time. Unfortunately, contamination of the milling powders can increase and undesirable phases can form if powders are milled past steady state [31].

The grinding medium is of the same material of the milling container to prevent contamination of powders. High density medium is chosen so the impact force on the powders is large enough to fracture and cold-weld the powders. The size of the grinding medium influences the milling efficiency. Instances in literature show different sized grinding medium create the highest collision energy [29]. This increase in collision energy and variation in medium size prevent excess wear of the medium and milling container while also avoiding contamination of the powders. However, the different in ball size is shown to lead to lower powder yield.

The BPR has a significant effect on the milling time to achieve particular phases of starting powders. Higher BPR leads to shorter milling time required because of the increase of collisions over time increases allowing more energy to be transferred to the powder system. The BPR, milling time, and milling speed effects the extent the milling container is filled. Mixing and alloying of the powders result due to the impact forces experienced on them. Thus, the powder and medium used for the MA process must be enough to have increased powder yield, but not so much there is not enough space for the impact to occur.

The milling atmosphere can cause contamination to the powders, therefore, the MA process either has milling containers vacuum or filled with an inert gas (argon or nitrogen). Nitrogen has been found to react with metal powders and to prevent contamination, high purity argon is most common to prevent oxidation and contamination of the powders.

As stated earlier, alloying of the powders can only take place with constant fracturing and cold-welding. With high milling speed, large BPR, increased milling time, etc., cold welding of the powders overwhelms the process. The cold-welding takes place due to the heavy plastic deformation. To promote equal fracturing and cold-welding a lubricant, known as a process control agent (PCA) is used. The PCA coats the surface of the powders and minimizes the cold-welding between the particles, medium, and milling container. The choice of PCA depends on the powders and the desired final product. The type and amount of PCA influences the final powder size and powder yield.

\subsubsection{Parameter Process Controls of Ball Milling}

The Taguchi technique is shown to provide an efficient and systematic approach to determine the optimal parameters in a manufacturing process. The main parameters of the planetary ball milling are the BPR, ball size, type of milling medium, volume of medium and the rotation speed. These parameters primarily effect the size, morphology and microstructure of the powders that are being mechanically alloyed, which Zhang, Zhu, 
and Wang examined with WC-Co powder. Table 2-3 shows the factors considered for optimizing the ball milling process and the median size of the composite particle, specific surface area, and microstructure was determined.

Table 2-3. Assignment of factors and levels of $L_{16}\left(4^{5}\right)$ orthogonal array[34]

\begin{tabular}{llllll} 
Factors & A & B & C & D & E \\
\cline { 2 - 6 } & $\begin{array}{l}\text { Weight ratio } \\
\text { of balls to } \\
\text { powder }\end{array}$ & $\begin{array}{l}\text { Balls } \\
\text { Size } \\
(\mathrm{mm})\end{array}$ & $\begin{array}{l}\text { Types of } \\
\text { milling } \\
\text { medium }\end{array}$ & $\begin{array}{l}\text { Volume } \\
\text { Of } \\
\text { Medium } \\
(\mathrm{ml})\end{array}$ & $\begin{array}{l}\text { Rotation } \\
\text { Speed } \\
\text { (RPM) }\end{array}$ \\
\hline 1 & $5: 1$ & 6 & $\begin{array}{l}\text { Distilled } \\
\text { Water }\end{array}$ & 0 & 100 \\
2 & $10: 1$ & $6+8$ & Ethanol & 4 & 150 \\
3 & $15: 1$ & $8+12$ & $\begin{array}{l}\text { Acetone } \\
\text { Ethanol }\end{array}$ & 8 & 12 \\
4 & $20: 1$ & $12+18$ & $\begin{array}{l}\text { Water } \\
\text { solution }\end{array}$ & & 200 \\
& & & &
\end{tabular}

Given the factors, the results of the grain size, median powder size, and specific surface area are shown in Table 2-4. From Table 2-4 higher BPR can improve the reduction of particle size and larger balls provide higher energy within the rotation of the ball mill, leading to higher impact energy to improve deformation and fracture of the WC particles. However, the authors found the best particle size was obtained with a ball size combination of $8+12 \mathrm{~mm}$ balls; due the size of the medium balls, cold-welding of the particles were limited and prevent agglomeration of the powders. Also, due the larger balls higher impact energy, the powders had less particle size reduction and, in fact, there was an increase in cold-welding and agglomeration [34]. Based on the results, it can be found the volume of the milling medium and the rotation speed of the ball mill offers the largest contributions to the particle size and grain size.

To prevent contamination of ODS powders during ball milling, a process control agent (PCA) will be used in place of the milling media introduced in this text. PCA, stated earlier, allows equal fracturing and cold-welding of the MA process. The type and amount of PCA depends on the starting mix of powders. Ramezani and Neitzert demonstrate the effects of PCA on mechanically alloyed aluminum powder through planetary ball milling. Use of an orthogonal array matrix relates the average grain size and surface depending on the changes of BPR, medium size, PCA, volume of medium, and the rotation speed used for tungsten carbide (WC) powders.

Table 2-4. $L_{16}\left(4^{4}\right)$ orthogonal array matrix and response of grain size, D50 and SSA[34]

\begin{tabular}{lllllllll}
\hline $\begin{array}{l}\text { Experiment } \\
\text { number }\end{array}$ & A & B & C & D & E & Results & & \\
\cline { 2 - 8 } & 1 & 2 & 3 & 4 & 5 & $\begin{array}{l}\text { Grain size of } \\
\text { WC }(\mathrm{nm})\end{array}$ & $\begin{array}{l}\text { D50 } \\
(\mathrm{nm})\end{array}$ & $\begin{array}{l}\text { SSA } \\
\left(\mathrm{m}^{2} / \mathrm{g}\right)\end{array}$ \\
\hline
\end{tabular}




\begin{tabular}{lllllllll}
\hline 1 & 1 & 1 & 1 & 1 & 1 & 104.6 & 2656 & 0.267 \\
2 & 1 & 2 & 2 & 2 & 2 & 46.5 & 1413 & 0.479 \\
3 & 1 & 3 & 3 & 3 & 3 & 59.4 & 1392 & 0.605 \\
4 & 1 & 4 & 4 & 4 & 4 & 33.3 & 592 & 1.269 \\
5 & 2 & 3 & 2 & 3 & 4 & 23.4 & 693 & 1.069 \\
6 & 2 & 2 & 1 & 4 & 3 & 23.1 & 564 & 1.030 \\
7 & 2 & 1 & 4 & 1 & 2 & 45.1 & 3586 & 0.192 \\
8 & 2 & 4 & 3 & 2 & 1 & 48.9 & 1938 & 0.422 \\
9 & 3 & 1 & 3 & 4 & 2 & 23.8 & 698 & 0.85 \\
10 & 3 & 2 & 4 & 3 & 1 & 31.8 & 837 & 0.747 \\
11 & 3 & 3 & 1 & 2 & 4 & 22.5 & 569 & 1.067 \\
12 & 3 & 4 & 2 & 1 & 3 & 17.3 & 2676 & 0.251 \\
13 & 4 & 1 & 4 & 2 & 3 & 23.2 & 866 & 0.721 \\
14 & 4 & 2 & 3 & 1 & 4 & 11.1 & 1503 & 0.433 \\
15 & 4 & 3 & 2 & 4 & 1 & 35.7 & 769 & 0.856 \\
16 & 4 & 4 & 1 & 3 & 2 & 26.5 & 667 & 0.941 \\
\hline
\end{tabular}

The Taguchi method used in the study for WC powders show higher impact energy (rotation speed) and a BPR of $15: 1$ is suitable to obtain suitable grain size and particle size. The medium or ball size distribution also shows a combination of large and medium size balls are best for achieving suitable results.

\subsection{Additive Manufacturing (AM) Techniques}

AM processes has high degree-of-freedom and allows product customization. Since there are several ways to deposit materials and different energy sources high energy electron beam source can be used. The systems are categorized into three different types according to the material feeding: powder bed systems, powder feed systems, and wire feed systems[35]. 


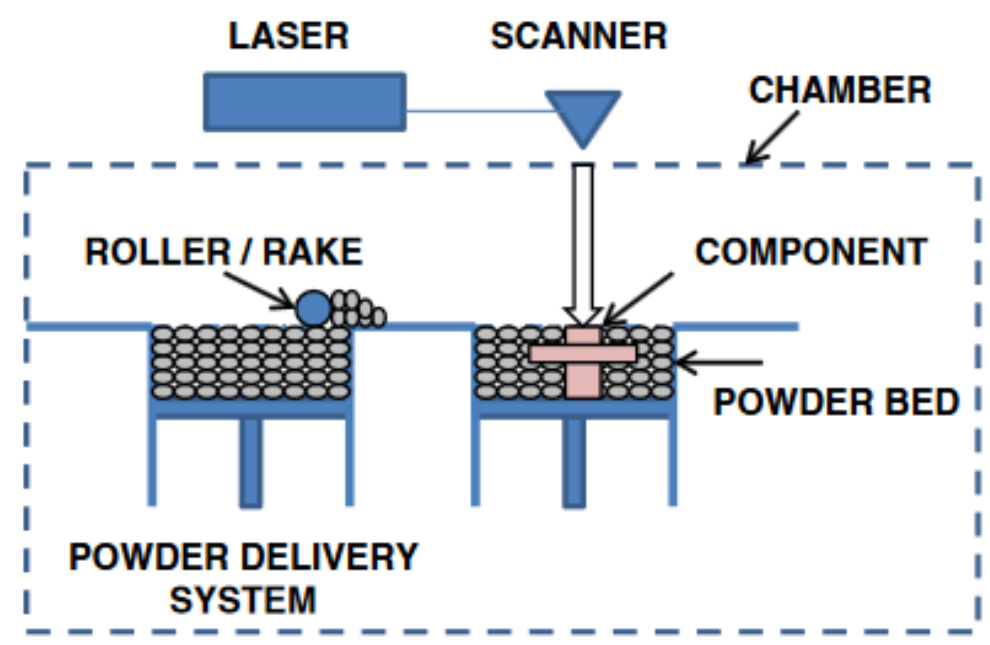

Figure 2-4.Schematic of general powder bed system [35]

\subsubsection{Powder bed systems}

Figure 2-4 shows a schematic diagram of a typical powder bed system. A roller rakes powder from the reservoir across the work area. The energy source is then delivered to the surface of the bed and the powder is melted or sintered into the designated shape. The process of racking and melting/sintering the feed is repeated until a solid part is formed. The workpiece of powder bed systems has higher resolution features so that the dimension of the work piece can be produced precisely. The EOS M290 is the most prevailing powder bed system, fully commercialized and used by many industries for custom tool parts and research purposes [36].

\subsubsection{Powder feed systems}

Unlike powder bed systems, powder feed systems use a nozzle to transfer powders onto the build surface of the workpiece. Shown in Figure 2-5, the energy source melts layers of the powder into the shape desired and a 3D part is formed with repetition. The workpiece either remains static and the powder nozzle moves or the work piece moves while the powder nozzle is in a fixed position. The powder feed systems are known to have a larger build volume, Optomec450 LENS (Laser Engineered Net Shaping-LENS) as an example, which has $1.2 \mathrm{~m}^{3}$ build volume. Powder feed systems are also capable of refurbishing and repairing damaged parts of a workpiece. LENS is the most popular direct energy deposition AM process for powder development and testing, making it suitable for research applications. 


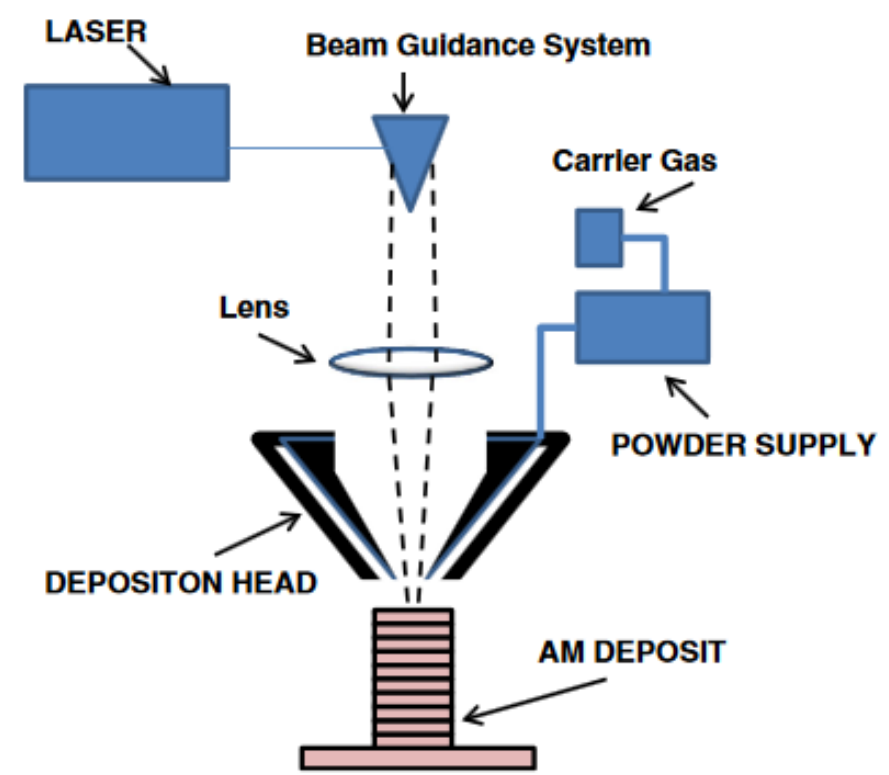

Figure 2-5. Schematic of general powder feed system [35]

\subsubsection{Wire feed systems}

Wire feed systems can use plasma arc as energy source instead of a high energy electron beam. At first, a single bead of material is deposited and upon subsequent passes is built upon to develop a 3D structure. Although wire feed systems have a high deposition rate, extensive machining and polishing are required.

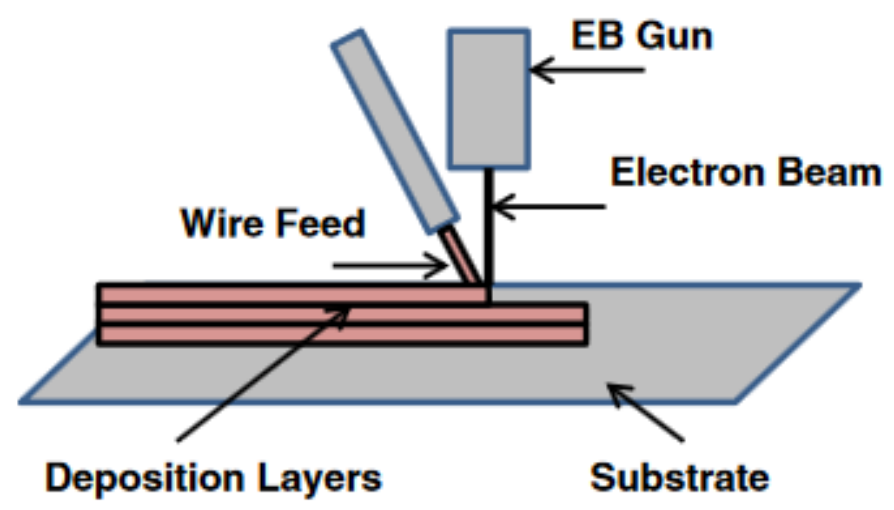

Figure 2-6. Schematic of general wire feed system [35] 
Selective laser melting (SLM) is another prominent powder bed AM technique that has higher precision and has better as-built surface quality when compared to electron beam and LENS. It has been fully commercialized (EOS Company) and becomes the leading technology in AM. The only restriction for the SLM is the size requirement of metal powder, which is lying in a narrow range between $40 \mu \mathrm{m} \sim 60 \mu \mathrm{m}$, and spherical in morphology. The purpose of setting this restriction is to ensure the powder bed has high packing density and the GA processed powder is the only way to provide the desired powder size and morphology. Other powder processes, such as the MA process, have very few reports in SLM application. However, another type of AM technique called Laser Cladding (LC) has a higher tolerance for powder shapes and sizes. The input energy of LC is much higher than LENS, and it is widely used in the industry. The objective in LC is to fuse an alloy layer or coating, onto the surface of a substrate. 


\section{THEORETICAL BACKGROUND}

\subsection{Dispersion Strengthening}

The addition of chemically stable secondary or ternary phase particle in metal matrices is used to strengthen alloys. The secondary phase particles added to the metal matrix are thermally and chemically stable, uniform, and fine. Thus, the particles are capable of pinning dislocation, grain boundaries, and sub-grain boundaries. In other words, prevention of grain growth and movement of dislocations is possible with the secondary phase particles, enhancing the mechanical properties of alloys. Also, the secondary phase particles have high melting points influencing dispersion strengthened metals to have high yield strength, tensile strength, creep, and corrosion resistance. Dispersion strengthening is also known and the theory of dislocation; the hardness of the secondary phase particles causes them to be obstacles for migration and movement of the dislocation line. As a result, high stress is needed to cause movement of the dislocation line. The particles can be identified as deformable and non-deformable, each interacting with dislocations differently.

\subsubsection{Orowan Theory}

In 1948, Orowan proposed a dispersion strengthened alloy under external loading, movement of any dislocation along the glide plane will interact with the non-deformable secondary phase particle. Increasing load increases the strength of the bowing and the dislocation line bypass the secondary phase particle [37]. As a result, the positive and negative dislocation interact and counteract one another, forming a dislocation ring and increasing the density of dislocation. The resistance force caused from the particle and dislocation line with respect to the tension of the dislocation line is:

$$
F=2 T \sin \theta
$$

Where $\theta$ is the angle of dislocation line when the secondary phase particle overlap. When the angle reaches 90 degrees, the dislocation ring becomes a semicircle; the maximum value of the resistant force, $F$, is found when the tension of the dislocation line is found to be:

$$
T=\frac{1}{2} G b^{2}
$$

Where $G$ is the shear modulus and $b$ is the burgers vector of dislocation. Given the balancing condition:

$$
F_{\text {max }}=\lambda b \tau_{c}
$$

Where $T_{c}$ is the critical stress and $\lambda$ is the distance between the secondary phase particles; combing equations 3.3 and 3.1 with condition 3.2: 


$$
\tau_{c}=\frac{G b}{\lambda}
$$

As a result, when the external stress exceeds the critical stress, the dislocation line migrates and passes the secondary particle. Equation 3.4 shows an inverse proportionality to critical stress and the distance between the secondary particles. Thus, smaller distance result in alloys with higher yield strength.

\subsubsection{Ansell-Lenel Theory}

Given the secondary phase particle is deformable, Ansell and Lenell proposed the dislocation line will cut the particle instead of passing or encircling it. The crack of the secondary phase particle which causes the dislocation pile up is used as the yield criteria [38]. The applied stress on the particle is equal to the fracture stress of the particle. The dislocation will move as a pattern, causing the material to yield. The cracking or fracturing of the secondary phase particle consumes the driving force of dislocation movement and increases the dislocation resistance. Thus, the critical stress is defined as:

$$
\tau_{c}=\sqrt{\frac{G b G^{*}}{2 \lambda C}}
$$

Where $G^{*}$ is the shear modulus of secondary phase particle and $C$ is a constant.

\subsection{Elemental composition and effects on ODS Ni-based Superalloys}

Nickel has high chemical and structural stability which enables it to maintain a facecenter-cubic (fcc) lattice structure from room temperature to the melting point. Also, the $\mathrm{Ni}$ matrix is capable of forming alloys with other elements through solid solution strengthening, which allows for $\mathrm{Ni}$ to be the base of an alloy system. More importantly, $\mathrm{Ni}$ forms strengthening $Y^{\prime}$ phase with L12 structure by interacting with other metal elements in the system during aging processing [39]. All Ni-base alloys contain this phase as the matrix and it is also the element of forming the $\mathrm{y}$ matrix and $\mathrm{Y}$ " phase with $\mathrm{Nb}$ and $\mathrm{Ta}$.

Chromium is the solid solution strengthener in ODS Ni-based superalloys because of its capability to enhance the corrosion resistance and high-temperature oxidation resistance. $\mathrm{Cr}$ could form a densified $\mathrm{Cr}_{2} \mathrm{O}_{3}$ oxide film on the surface of metals to improve the passivation ability of metals. However, high content of $\mathrm{Cr}$ promotes the formation of a phase, which undermines the ductility of alloys. Considering the corrosion resistance and ductility of ODS alloys, Cr was chosen as $20 \mathrm{wt}$ \% in this research.

Tungsten is the element to facilitate the solid solution strengthening in ODS alloys. According to published results, the creep strength of ODS alloys increases with W contents. However, W concentration over $3 \mathrm{wt}$. \%, cause saturation in the matrix leading W to cause unstable phases such as Laves phase, weakening the creep strengths of ODS super alloys. As a result, the concentration of $\mathrm{W}$ was chosen to be $3 \mathrm{wt}$. \% in this study. 
Aluminum form the $\mathrm{Y}$-NiAl matrix. The material is also capable of providing oxidation resistance with the formation of a stable $\mathrm{Al}_{2} \mathrm{O}_{3}$ oxide later on the surface of ODS $\mathrm{Ni}$-based superalloys. It is also a booster for the $\mathrm{Y}^{\prime}$ precipitates which enhance the mechanical properties of Ni-based superalloys. Unfortunately, experimental studies have shown that Al composition over 5 wt. \%, causes y' phase to mismatch with the y matrix.

Yttrium Oxide concentration over 0.2 wt. \% shows apparent strengthening of metals. Higher than $0.5 \mathrm{wt}$. \%, shows occurrences of micro voids along the grain boundary, increasing the diffusion of oxygen and decreasing the oxidation resistance of alloys. 


\section{MATERIALS AND EXPERIMENTAL METHODS}

\subsection{Experimental Materials}

Under inert atmosphere, raw elemental powders are mixed at specified weight percentages and sealed for further processing; Table 4-1 shows the raw elemental powders average size distribution and weight percentage.

Table 4-1.1 Breakdown of elemental powders used to produce ODS

\begin{tabular}{cccc} 
Chemical Name & Chemical Formula & $\begin{array}{c}\text { Average Particle } \\
\text { Size }\end{array}$ & $\begin{array}{c}\text { Powder Weight } \\
\text { Distribution (\%) }\end{array}$ \\
\hline Yttrium (III) Oxide & $\mathrm{Y}_{2} \mathrm{O}_{3}$ & $25-50 \mathrm{~nm}$ & 1.5 \\
Tungsten & $\mathrm{W}$ & $<1 \mu \mathrm{m}$ & 3 \\
Chromium & $\mathrm{Cr}$ & $<10 \mu \mathrm{m}$ & 20 \\
Aluminum & $\mathrm{Al}$ & $4.5-7 \mu \mathrm{m}$ & 5 \\
Nickel & $\mathrm{Ni}$ & $3 \mu \mathrm{m}$ & 70.5
\end{tabular}

$\mathrm{Y}_{2} \mathrm{O}_{3}$ was purchased from supplier Sigma Aldrich, which the remaining powders (Ni, Cr, Al, and W) were purchased from Alfa Aesar. The average particle size of the constituent element powders are less than 10 micrometers, while the $\mathrm{Y}_{2} \mathrm{O}_{3}$ has an average particle size between 25 and 50 nanometers. Given the powder weight distribution, the raw elemental powders were mixed, sealed in bottles with Argon gas, and stored in a glove box of Argon atmosphere until further processing. Stearic acid, $\mathrm{CH}_{3}$ $\left(\mathrm{CH}_{2}\right)_{16} \mathrm{CO}_{2} \mathrm{H}$, will also be used as a lubricant for the powders during the ball milling (BM) process in order to control the size and particle distribution of the powders. Figure 4-1 demonstrate the particle distribution, average particle size and particle morphology of the elemental powders before mixing. It is evident the morphology of the individual particles varies widely; the aluminum powders appear to have a spherical shape, the nickel powders appear irregularly round, and the remaining elemental powders appear flaky. As stated earlier, the goal is not only to produce uniform ODS powders using a combination of MCB and BM, but fabricate powders with suitable size distribution and spherical in 
shape for use in AM techniques. The morphology and size of the elemental powders allows for better control of the fabrication techniques.
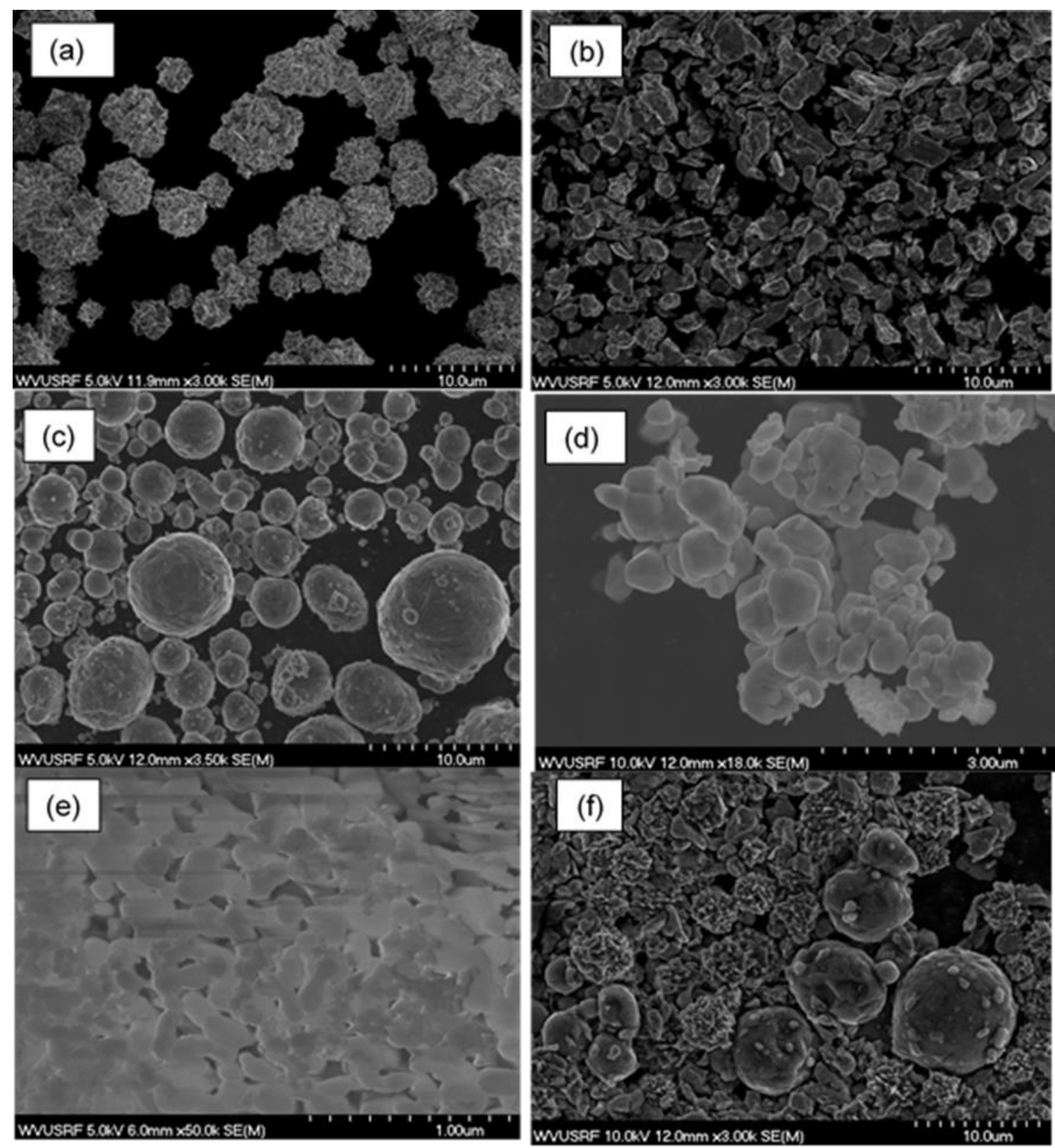

Figure 4-1. SEM Micrographs of (a) Ni powder, (b) Cr powder, (c) Al powder, (d) W powder, (e) Y2O3 and (f) As-blended powders 


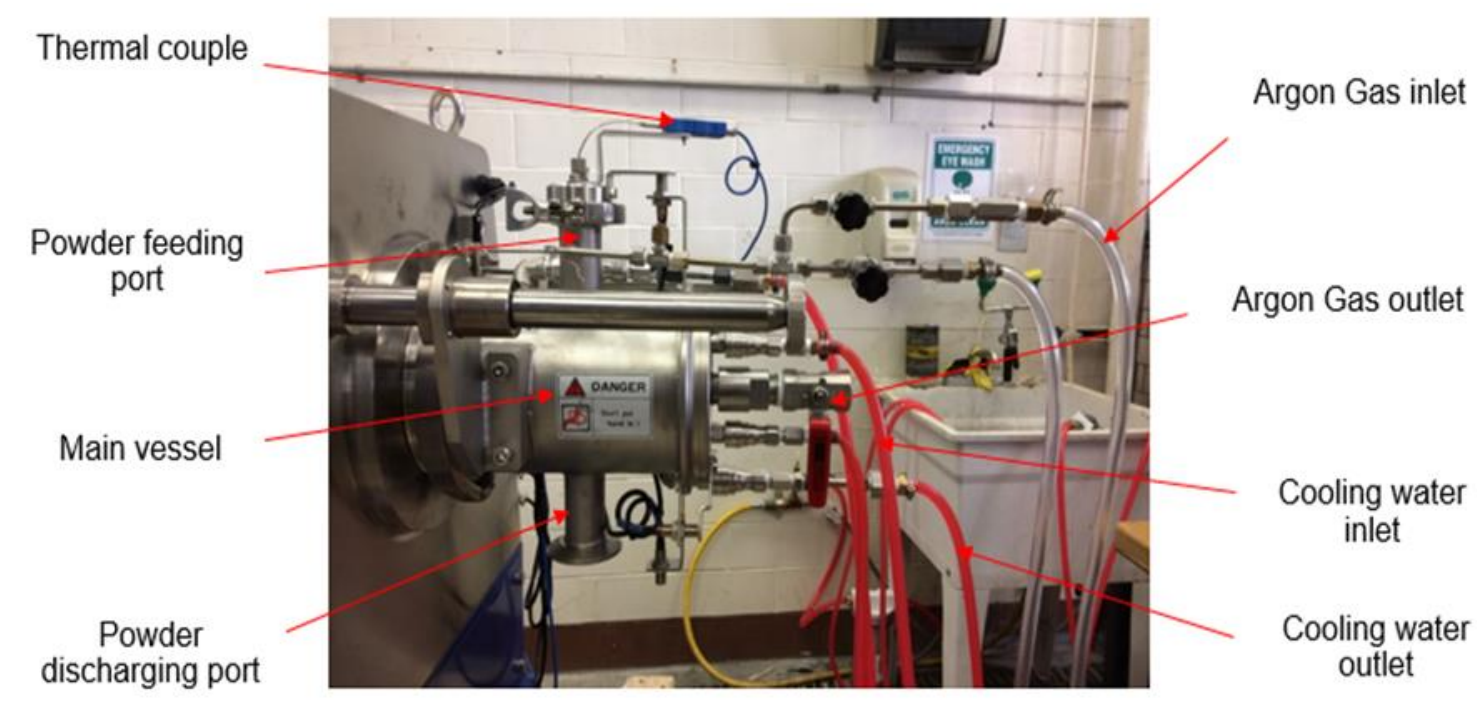

Figure 4-2. HOSOKAWA NOBILTA-130 MCB Machine

\subsection{Experimental Methods and Instruments}

The as blended powders were initially processed using the MCB technique and further processed through ball milling. Optimization and studies done for the MCB technique was done in cooperation of Sizhe Huang, a former Master student under Dr. Bruce Kang's supervision. Sizhe Huang shares any credit shown from the results gathered with results obtained from MCB.

\subsubsection{Mechano-Chemical Bonding (MCB) Process}

The MCB machine used in this research if the NOBILTA-130 model from HOSOKAWA Micron LTD. The machine has a capacity of 0.5 liters and between 300-400 grams of powders can be produced per run. Figure 8 is a simple schematic of the MCB machine and labels key components. Argon gas is used to flush out air in the vessel and is done multiple times to ensure an inert atmosphere. The as-blended powders are processed for 1 hour at $4000 \mathrm{rpm}$ which allows the $\mathrm{Y}_{2} \mathrm{O}_{3}$ nanoparticles even distribution and bonding on the $\mathrm{Ni}$ and $\mathrm{Cr}$ host particles.

\subsubsection{Mechanical Alloying via Ball Milling (BM)}

The MCB produced powders are further processed by use of a planetary ball milling machine. The SFM-1 (QM-3SP2) High Speed Shimmy Ball Mill was used with 4 stainless steel balls, stainless steel grinding medium of various sizes, and a process control agent (PCA). As stated previously, the grinding medium sizes were used based on previous literature and trial and error. All experiments conducted using the planetary ball mill used $4-20 \mathrm{~mm}$ balls, $4-15 \mathrm{~mm}$ balls, $2-10 \mathrm{~mm}$ balls, and $2-5 \mathrm{~mm}$ balls. The primary parameters of planetary ball milling that affects average particle size, particle size 
distribution and morphology are milling energy, milling time, and ball-to-powder (BPR) ratio. The control of the ball milling procedure was done by controlling these parameters and characterizing the morphology and microstructure. Table 4-2 below, shows the changes in parameters and will be labeled as configurations for simplification. It was shown higher BPR yields shorter time for powders to reach steady state conditions and results in a faster refinement of crystallite size, while higher rotation leads to higher energy input into the powder. It is also important to focus on the yield given per run and time taken to complete a run when optimizing the ball milling technique.

Table 4-2. Configuration of planetary ball milling parameters.

\begin{tabular}{llll} 
Configuration & Speed $(\mathrm{rpm})$ & BPR & Time (hours) \\
\hline C1 & 300 & $15: 1$ & 15 \\
C2 & 300 & $30: 1$ & 15 \\
C3 & 300 & $30: 1$ & 45 \\
C4 & 400 & $15: 1$ & 15 \\
C5 & 400 & $15: 1$ & 25 \\
C6 & 400 & $15: 1$ & 30 \\
C7 & 400 & $15: 1$ & 40
\end{tabular}

\subsubsection{Particle Size Distribution}

The size distribution of each configuration will be analyzed using a Malvern PANalytical Master Sizer 2000 particle analyzer. Distilled water was used to disperse the powder in the laser system and also used to purge the chamber of the analyzer after each test.

\subsubsection{Laser Engineering Net Shaping (LENS)}

The optimized MCB+BM powders were sent to the University of Pittsburgh for additive manufacturing on a LENS 450 system. Three input laser energies were selected $(200 \mathrm{~W}, 250 \mathrm{~W}$, and $275 \mathrm{~W})$, two laser scanning speeds $(33 \mathrm{~mm} / \mathrm{s}$ and $10 \mathrm{~mm} / \mathrm{s})$, two powder feeding rated (8 rpm and $11 \mathrm{rpm}$ ), and two flat substrates were adapted (MAR247R and Stainless Steel).

\subsubsection{Finite Element Analysis}

Dassault Systemes SIMULIA Abaqus CAE will be used to determine the mechanical properties of spherical, elliptical, and irregularly shaped particles and a shape factor will be derived. Lastly, simulation results will be compared to experimental results done using in-house micro-indentation technique on spheres with known properties, such as 316 stainless steel and 1100 aluminum alloy. Using a load based multiple-partial unloading micro-indentation technique derived from Hertzian contact laws, the mechanical properties of the ODS particles will be determined. 2-node linear axisymmetric rigid link (RAX2) mesh was used to model a flat indenter and a 3-node linear 
axisymmetric triangle mesh (CAX3) was used to model the particle used for indentation. The particles were indented to an overall depth of $30 \mu \mathrm{m}$ over 10 steps and the unloading curves were used to determine the material properties.

\subsection{Characterization Methods}

Analysis of MCB only, MCB+BM, and AM produced ODS were subjected to analysis in order to quantify the morphology, average particle size, particle size distribution, and microstructure. Thus, X-ray Diffraction (XRD), Scanning Electron Microscopy (SEM), and Energy Dispersive Spectrums (EDS) were used to characterize the material for comparison.

\subsubsection{SEM}

The Hitachi S-4700 from SRF WVU will be used for observation of the morphology, average particle size, and particle distribution of the ODS powders after powder fabrication. The SEM will also be used to analyze the morphology of $y$ matrix and $y^{\prime}$ precipitates. The powdered samples were dispersed unto carbon tape on inspection and the as-printed AM samples were held with the conventional sample holder used for most SEM analysis.

\subsubsection{XRD}

The XRD analysis was done with a PANalytical X'Pert Pro with a Cu-K alpha radiation $(45 \mathrm{kV}, 40 \mathrm{~mA})$. The $\mathrm{XRD}$ patterns were recorded at room temperature with a range of $10^{\circ}$ to $90^{\circ}$.

\subsubsection{EDS}

EDS was utilized to analyze the distribution of elements in the cross section asprinted samples. Especially, point scan was used to pinpoint the location and analyze the distribution, density, and phases. Mapping was also employed to observe the element distribution. 


\section{RESULTS AND DISCUSSIONS}

\subsection{Analysis of Fabricated Powder}

\subsubsection{SEM Analysis}

SEM imaging was conducted on the ODS powder samples in order to analyze the size, morphology, and size distribution under each configuration. Figure 5-1 and Figure 5-2 demonstrates the size distribution of the ODS powders for different milling times and BPR for rotation speed of $300 \mathrm{rpm}$ and $400 \mathrm{rpm}$, respectively. Figure 5-1 uses a magnification of $x 450$ with a scale of $100 \mu \mathrm{m}$, whereas Figure 5-2 uses a magnification of x600 with a scale of $50 \mu \mathrm{m}$. Figure 5-1 (a) and 5-2 (b), corresponding to C1 and C2 show small difference in the size distribution of the ODS powders; given a constant speed of $300 \mathrm{rpm}$ and milling time of 15 hours, doubling the BPR from 15:1 to $30: 1$ did not the particle distribution. However, C3, shown in Figure 5-1 (c), demonstrates agglomeration of the powders with a large BPR and milling time of 45 hours. Figures 5-2 (a)-(d) shows larger rotation speed of $400 \mathrm{rpm}$ leads to a uniform particle distribution, regardless of the BPR and milling time.

Using a higher magnification, the morphology of the ODS particles can be studied further. As seen in Figure 5-3 (a), the particles appear to be flat and flaky with little to no agglomeration of the particles. This is an indication of the beginning stages of the mechanical alloying process where the master particles are flattened to lamellar structures[29]. With the fixed time of 15 hours, Figure 5-3 (b) demonstrates higher BPR leads to less time for powders to reach steady state conditions. Although it is evident that C2 have not reached steady state, BPR is a major requirement for optimal Ni-based ODS powders. Milling time affects the size distribution of the particles as well. It is evident from Figure 5-3 (b) and 5-3 (c), with a constant speed of $300 \mathrm{rpm}$ and BPR of 15:1, the increase in hours from 15 to 45 lead to a decrease in the average particle size.

Given the PCA acts as a lubricant, stearic acid helps promote fracturing of the ODS particles, however, with increased milling time and operation, the temperature increases to a point where the stearic acid cannot control the cold-welding. In order to increase the average particle size, milling time, BPR, or rotation speed must be increased to obtain a suitable size distribution, average particle size, and spherical shape. An influential parameter, also factored in to the study, is the powder yield after ball milling. In order to increase the powder yield of the ODS powders, it was best to decrease the BPR, thus the milling time and rotation speed was optimized for desirable results. As observed in Figures 5-4 (a)-(d), a BPR of 15:1 and rotation speed of $400 \mathrm{rpm}$ lead to nearly spherical ODS particles with good size distribution. Figure 5-4 (a) still shows particles less than 5 $\mu \mathrm{m}$, which are troublesome for AM requirements. However, as milling time increased, as shown in Figures 5-4 (b)-(d), the smaller ODS particles agglomerate and form larger, nearly spherical particles. 

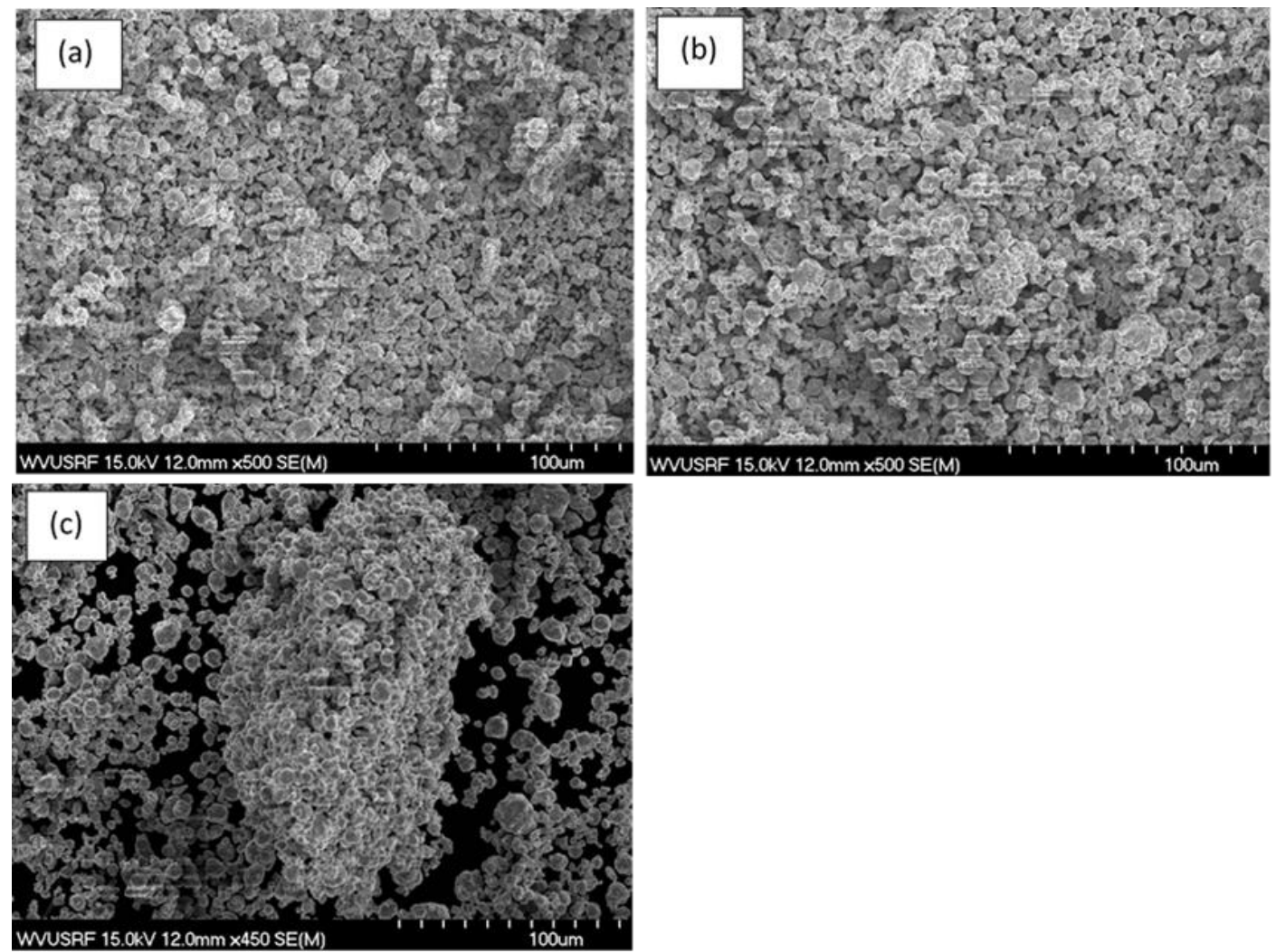

Figure 5-1. 300 rpm (a) BPR 15:1 15 hours (C1), (b) BPR 30:1 15 hours (C2), (c) 300 rpm BPR 30:1 45 hours (C3)
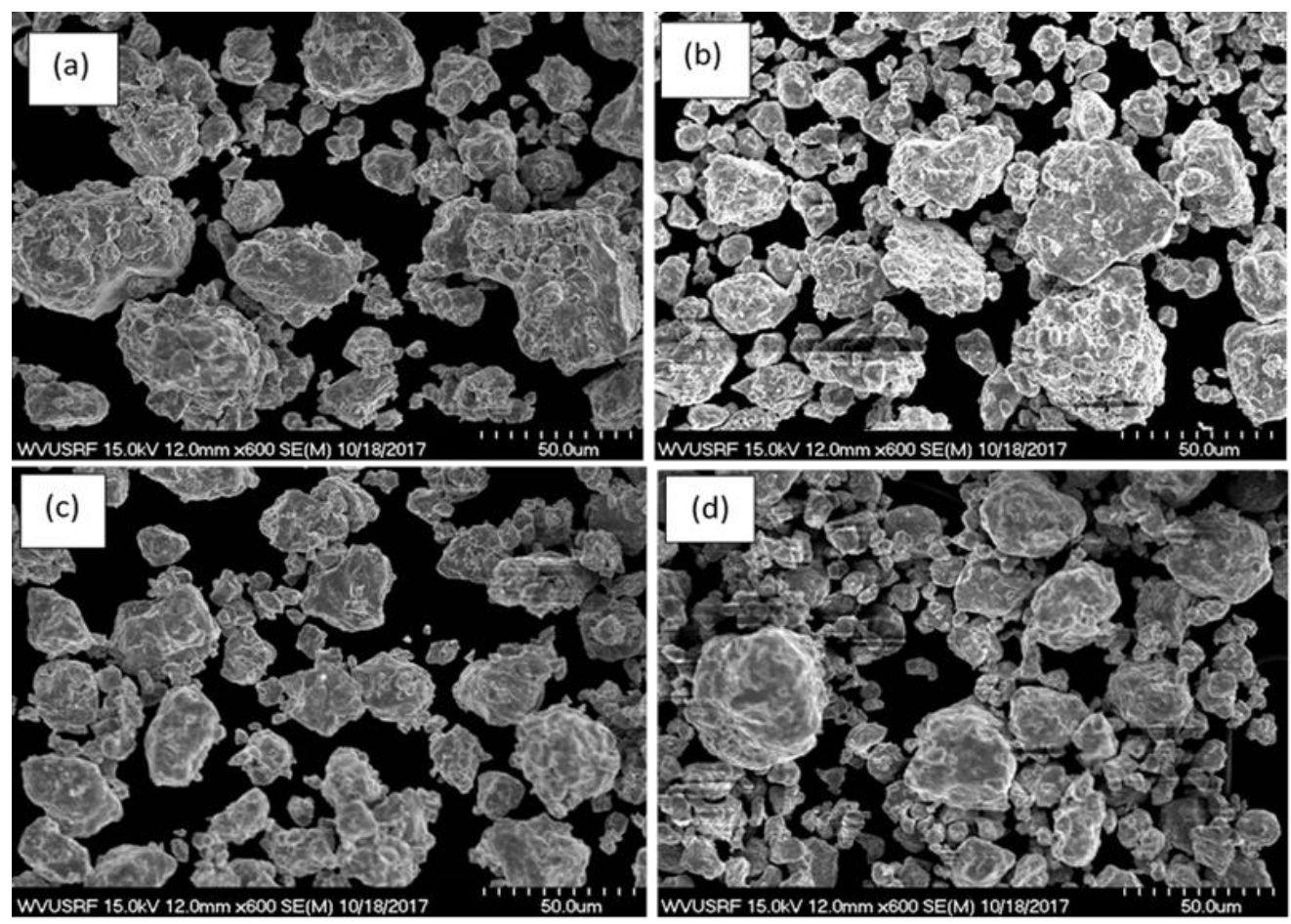

Figure 5-2. 400 rpm (a) BPR 15:1 15 hours (C4), (b) BPR 15:1 25 hours (C5), (c) BPR 15:1 30 hours (C6), (d) BPR 15:1 40 hours 

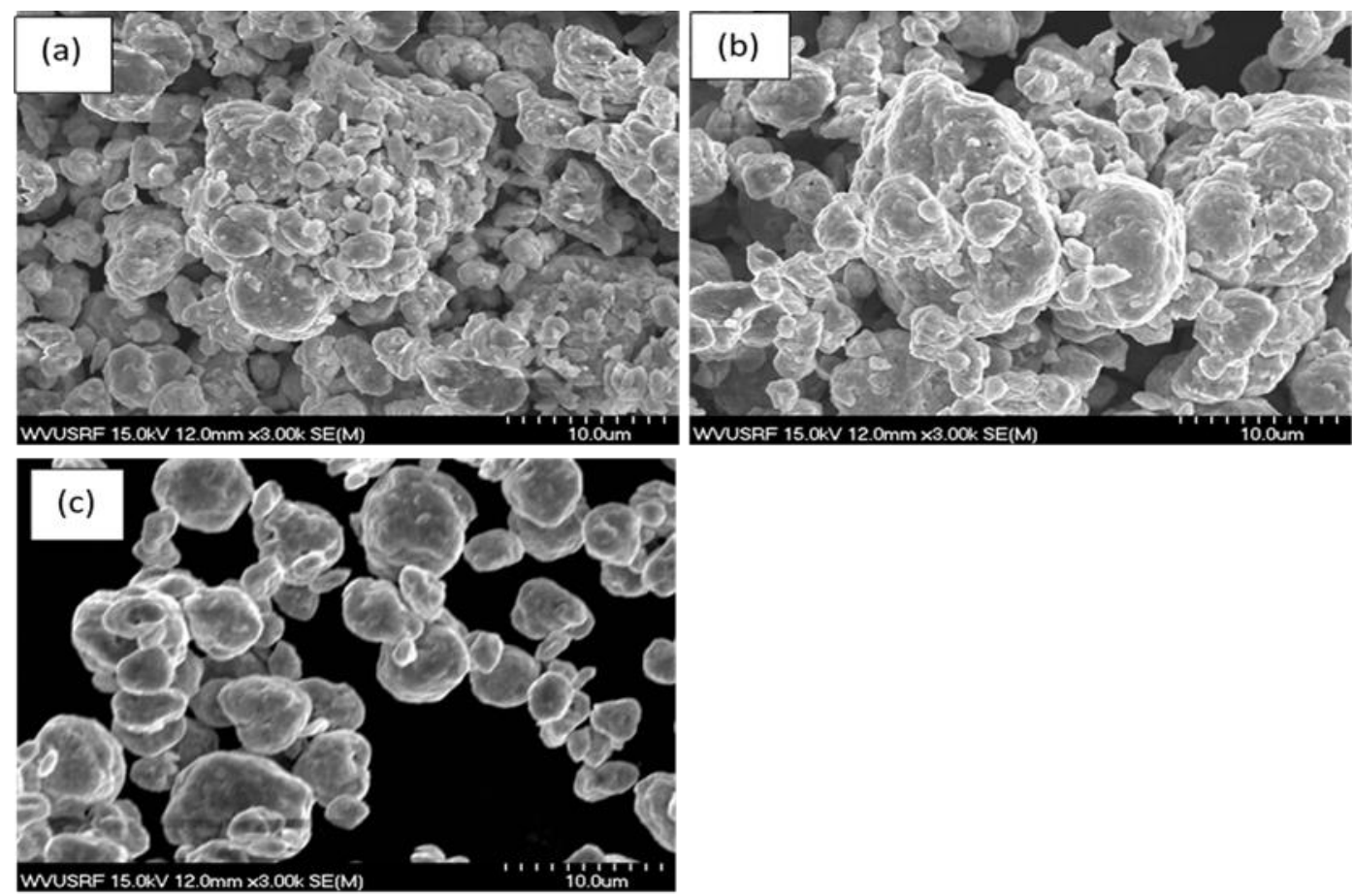

Figure 5-3. 300 rpm (a) BPR 15:1 15 hours (C1), (b) BPR 30:1 15 hours (C2), (c) 300 rpm BPR 30:1 45 hours (C3)
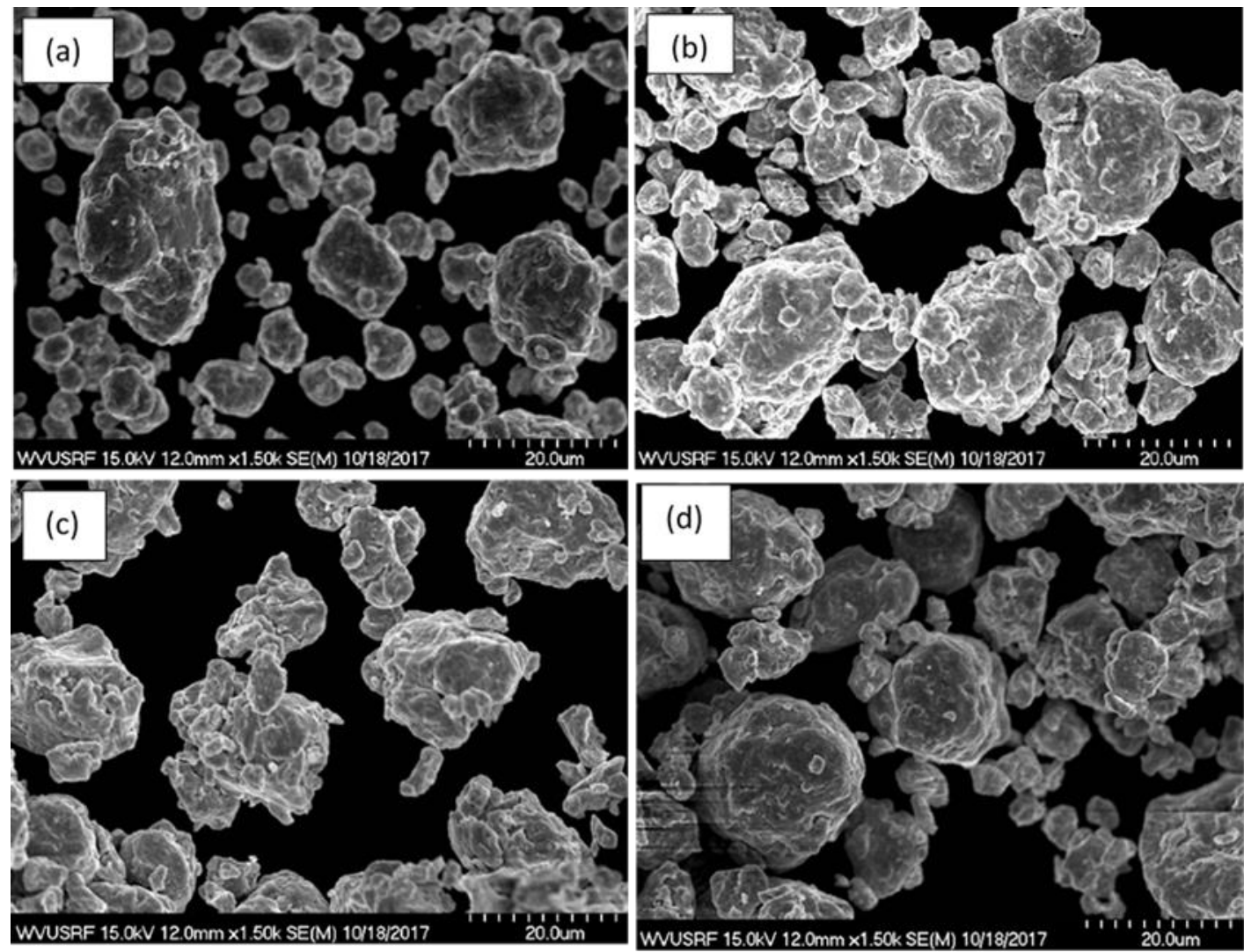

Figure 5-4. $400 \mathrm{rpm}$ (a) BPR 15:1 15 hours (C4), (b) BPR 15:1 25 hours (C5), (c) BPR 15:1 30 hours (C6), (d) BPR 15:1 40 hours 


\subsubsection{Size Distribution of Processed Powders}

Laser diffraction is widely used for measurement of average particle size distribution due to small operation time and repeatability. Using the Malvern PANalytical Master Sizer 2000, the processed powders size distribution was determined with results noted as $D(x)=$ size, where $D$ is the diameter of the particle measured by laser diffraction, $x$ is the mass percent of particles below the measured size. There are five variations of powder size distribution, shown in Figure 5-5, which published results have shown to describe powders[40]. The majority of commercial feedstocks used in SLM processes follow Gaussian distribution with the addition of removing powders below undesirable sizes via sieving operations. Gaussian distributions show the mean, median and mode coincide at the central point of the curve, whereas negatively skewed distributions shows a higher mean value (more coarse particles) and positively skewed distributions show a lower mean value (more fine particles) [40].
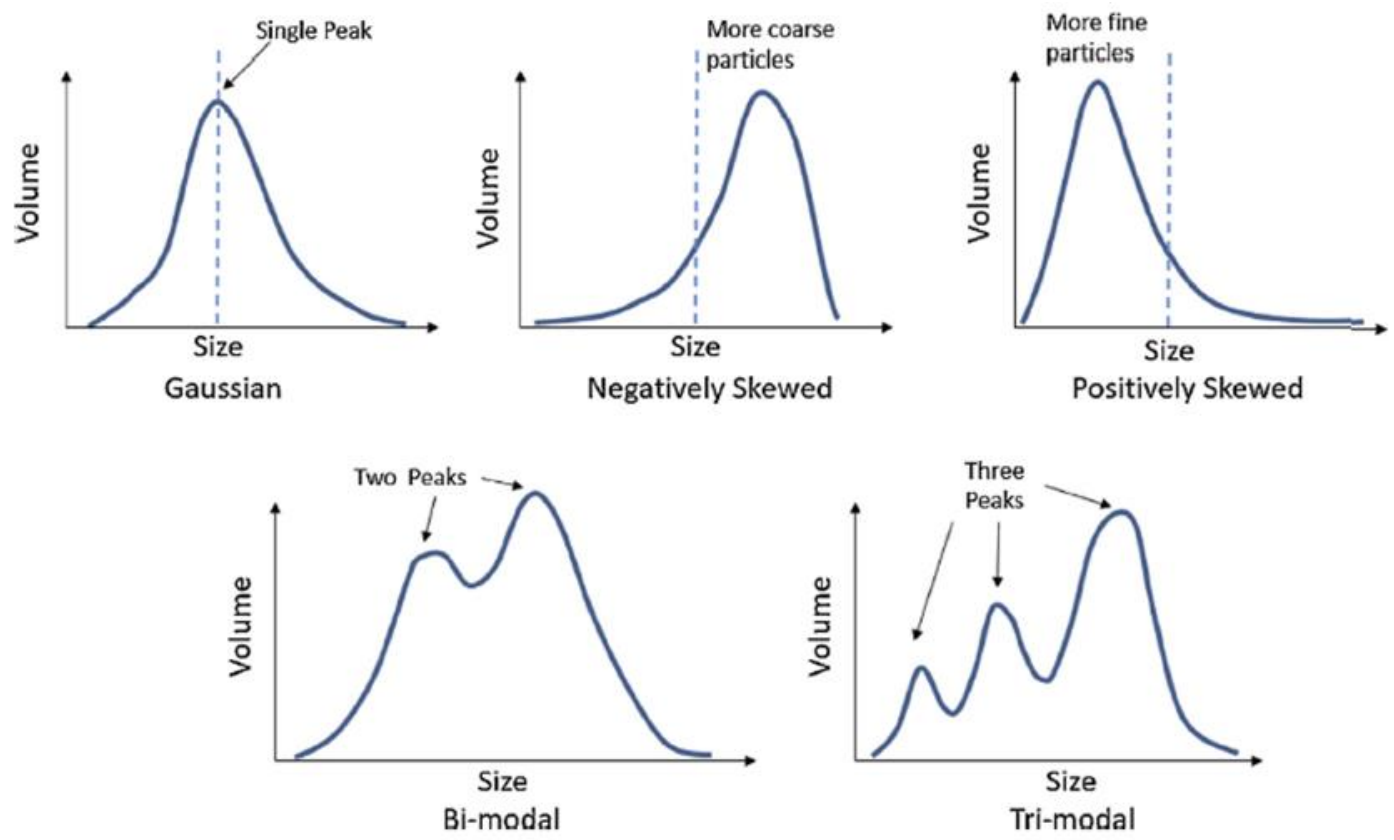

Figure 5-5. Variations of powder size distributions) [40]

Given the SEM images, it's desirable to control the process parameters of the BM technique with rotation speed of $400 \mathrm{rpm}$ and BPR 15:1. As stated, although a lower BPR provides less energy to the powdered particles, an increase in rotation speed will overcome the loss in energy and increase the yield at end of production run. Thus, study of the optimal milling time for the powders to reach steady state conditions, have uniform particle distribution, average particle size greater than $10 \mu \mathrm{m}$, and spherical shape becomes the next goal. Figures 5-6 - 5-9 show the powders processed by 
MCB+BM at 400 rpm, BPR 15:1, and milling times of 15, 25, 30, and 40 hours, respectively.
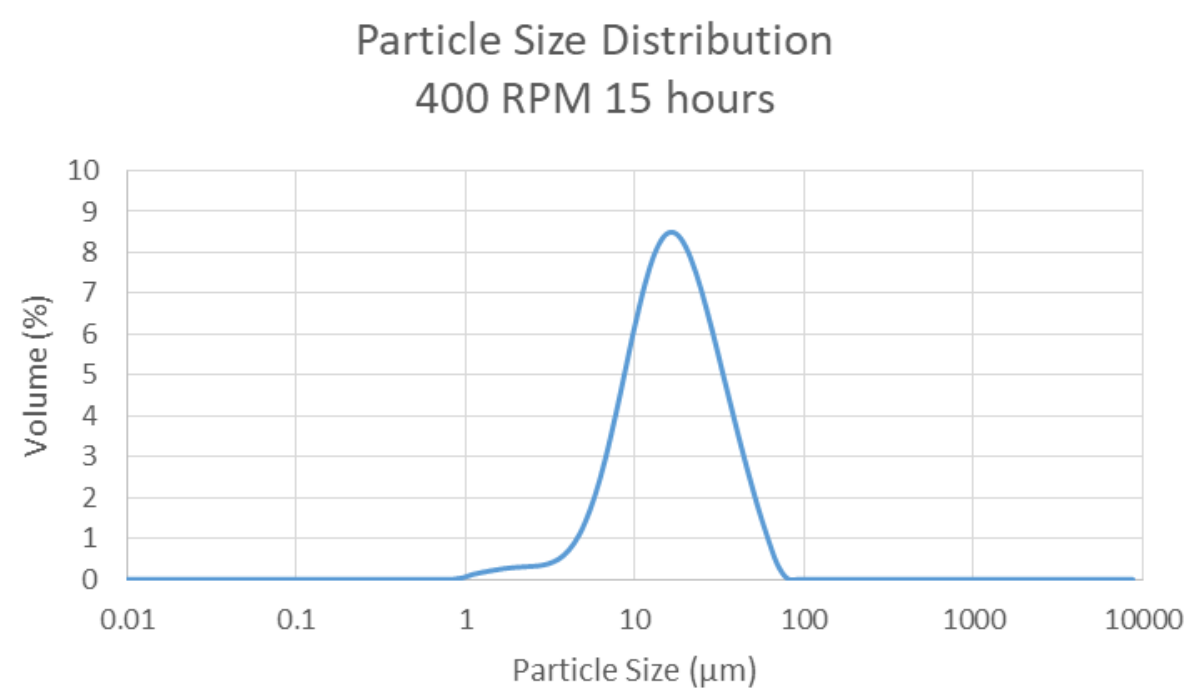

Figure 5-6. Powder size distribution of MCB+BM processed powder $400 \mathrm{rpm}$ BPR 15:1 15 hours $\mathrm{D}(0.1)=5.79 \mathrm{D}(0.5)=13.891 \mathrm{D}(0.9)=121.129$

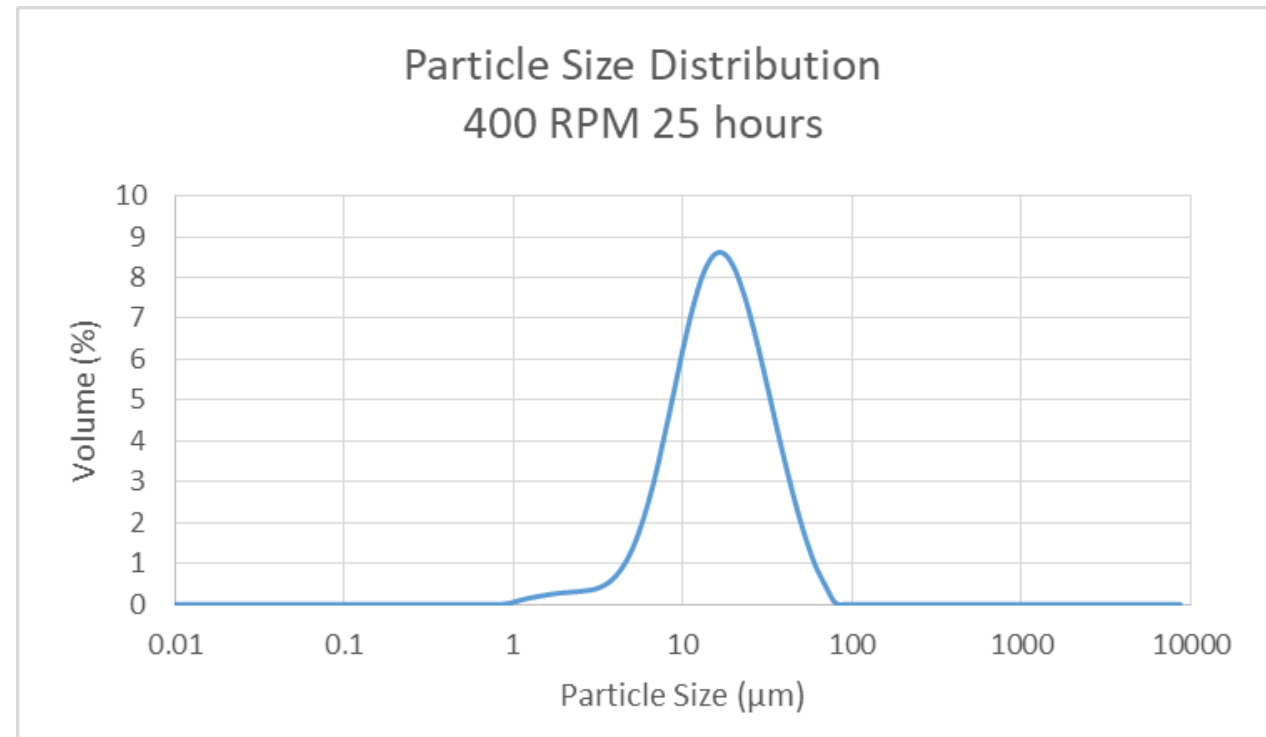

Figure 5-7. Powder size distribution of MCB+BM processed powder $400 \mathrm{rpm}$ BPR 15:1 25 hours $\mathrm{D}(0.1)=8.090 \mathrm{D}(0.5)=21.8 \mathrm{D}(0.9)=66.896$ 


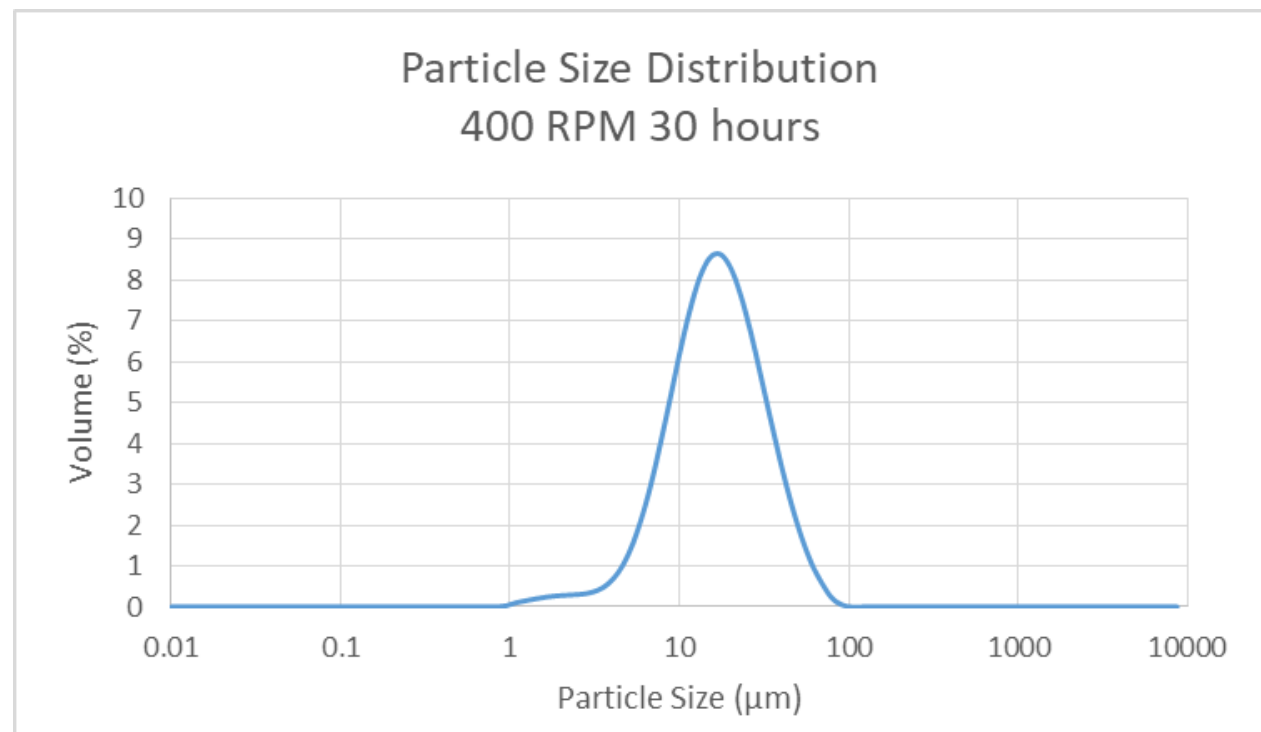

Figure 5-8. Powder size distribution of MCB+BM processed powder $400 \mathrm{rpm}$ BPR 15:1 30 hours $\mathrm{D}(0.1)=7.57 \mathrm{D}(0.5)=17.663 \mathrm{D}(0.9)=39.273$

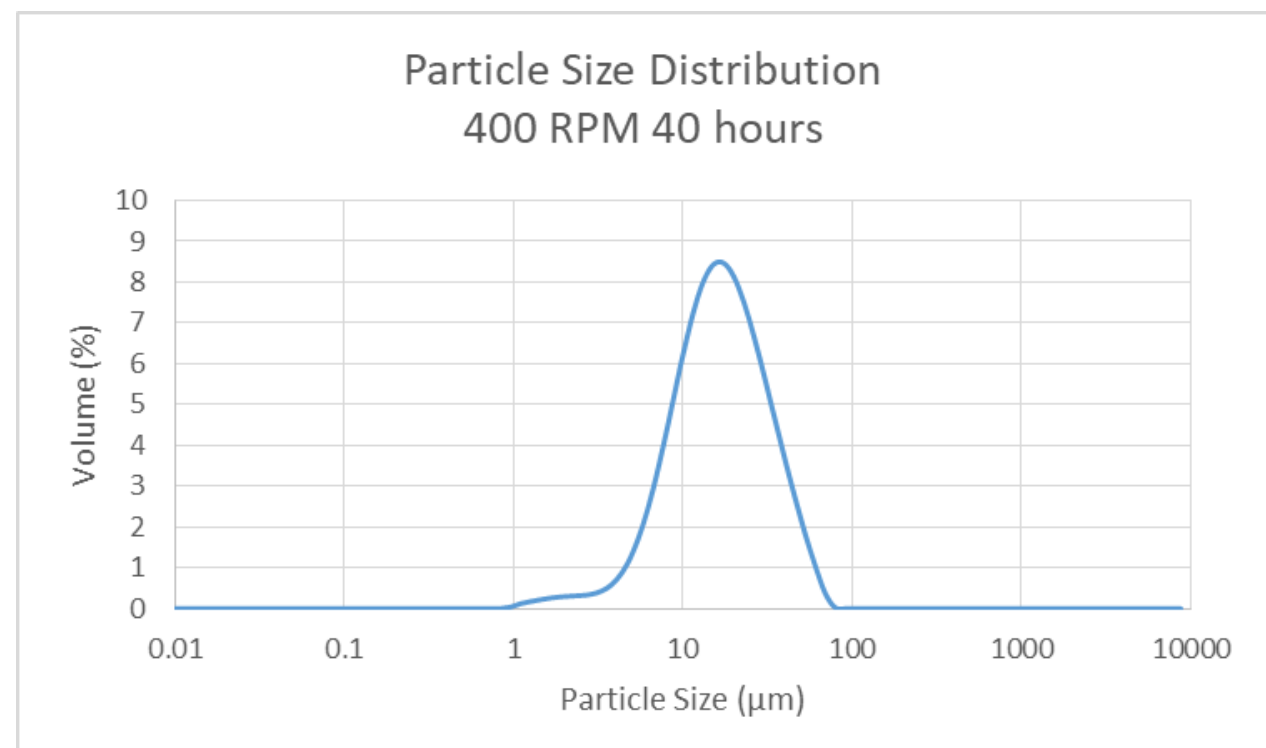

Figure 5-9. Powder size distribution of MCB+BM processed powder $400 \mathrm{rpm}$ BPR 15:1 40 hours $\mathrm{D}(0.1)=7.836 \mathrm{D}(0.5)=14.296 \mathrm{D}(0.9)=26.846$ 
As shown in Figures 5-6 -5-9, given a constant milling speed of $400 \mathrm{rpm}$ and BPR of $15: 1$, changes in milling time changed the average particle size and particle size distribution. The average particle size of C4, C5, C6, and C7 are $13.891 \mu \mathrm{m}, 26.800 \mu \mathrm{m}$, $17.663 \mu \mathrm{m}$, and $14.296 \mu \mathrm{m}$, respectively. Increasing the milling time from 15 hours to 25 hours drastically increased the average particle size by almost double. This is further emphasized in the SEM images, as the ODS particles not only increase in size, but the agglomeration of the particles is more evident. As the milling time increases from 25 hours to 30 hours, the average particle sizes decrease and continues to decrease up to 40 hours. 10 percent of the volume of the ODS particles also shows an increase in size from $5.790 \mu \mathrm{m}$ to $8.090 \mu \mathrm{m}$ with an increase in milling time. Further increase in milling time there is a slight decrease, which for 40 hours, 10 percent of the ODS particles are below $7.836 \mu \mathrm{m}$. Further evidence that an increase in the milling time allows for larger particle sizes and a suitable average particle size for AM applications.

To allow for flow ability the average particle size not only has to be above $10 \mu \mathrm{m}$, but there has to be uniform particle size distribution. C1 shows, with a milling time of 15 hours there are particles as large as $121.628 \mu \mathrm{m}$; however, as the milling time increases, the size of the larger particles received by the laser diffraction decreases to $26.896 \mu \mathrm{m}$ at 40 hours. As a result, it is clear the increased milling time provides a suitable average particle size for AM processes and a uniform particle size distribution. 


\subsubsection{XRD Analysis}

The processed powders were characterized by XRD using a PANalytical X'Pert PRO X-Ray diffractometer with $\mathrm{Cu}-\mathrm{Ka}$ radiation and spinner stage. The XRD patterns were recorded at room temperature with a step size of $0.0167^{\circ}$ with 40 second time period per step. The XRD spectrum of the as-received pure elemental starting powders are shown in Figure 5-10. Al powder shows major peaks around $38^{\circ}, 45^{\circ}$ and $65^{\circ}$; W powder shows major peaks around $40^{\circ}, 58^{\circ}$, and $73^{\circ}$; Ni powder major peaks are $45^{\circ}, 52^{\circ}$, and $78^{\circ}$. Lastly, $\mathrm{Y}_{2} \mathrm{O}_{3}$ and $\mathrm{Cr}$ share a major peak of $30^{\circ}$ and $45^{\circ}$, however, $\mathrm{Y}_{2} \mathrm{O}_{3}$ is a nanoparticle and display the line-broadening effect.

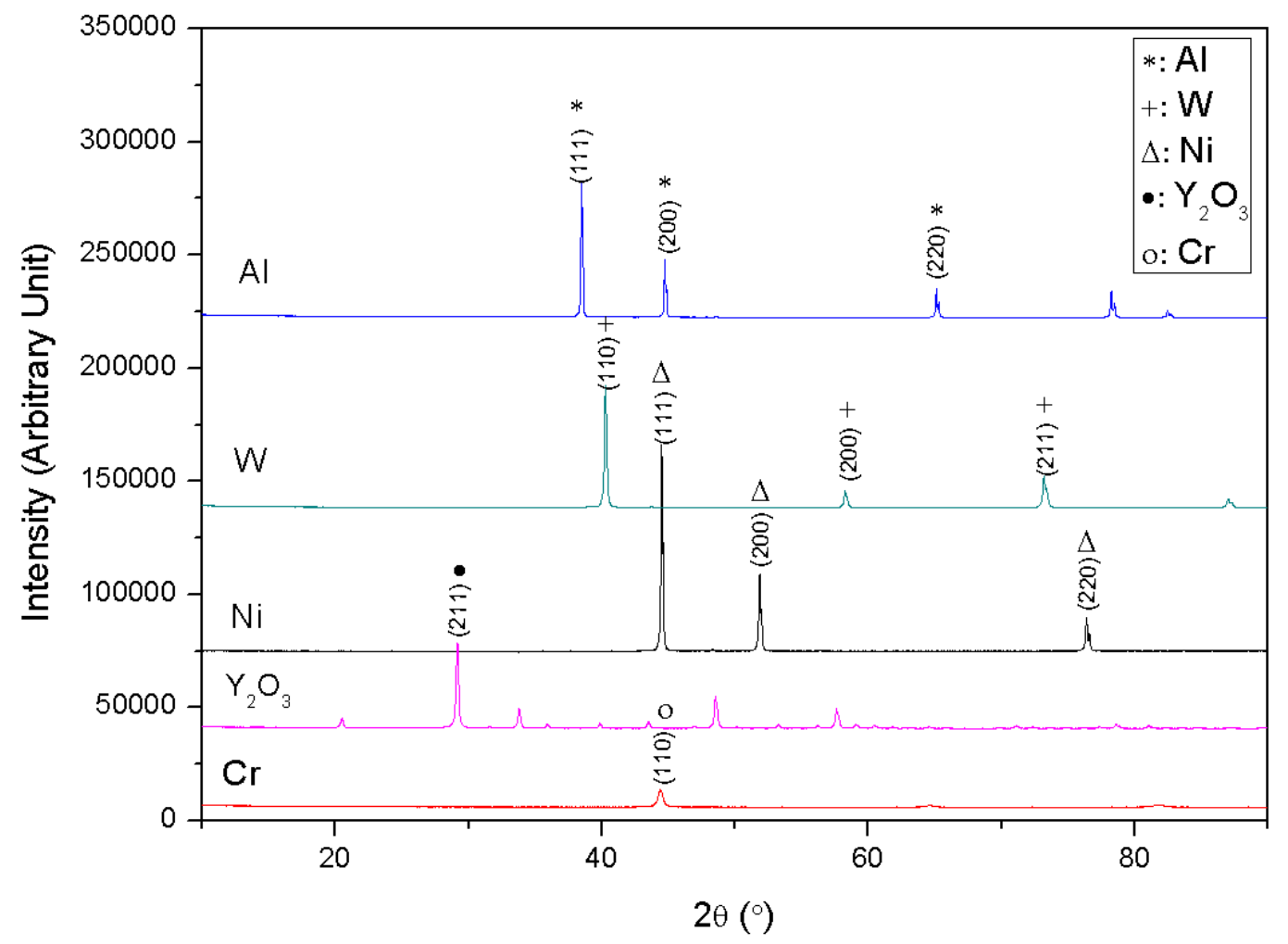

Figure 5-10.XRD patterns of pure elemental starting powders

The preliminary MCB only (4000 rpm for 1 hour) of the combined MCB + BM process shows a disappearance of the $\mathrm{Y}_{2} \mathrm{O}_{3}$ (211) peak. Thus, the $\mathrm{Y}_{2} \mathrm{O}_{3}$ is confirm to be uniformly distributed on the master particles as a thin film. Residual Al (111) and W (110) peaks are also still observed, implying the powders have not alloyed during the MCB process. However, evidence of the NiAl (110) peak is shown at about $44^{\circ}$, meaning the MCB only process promotes the beginning of alloy phase for the ODS Ni-base alloy. Unfortunately, $\mathrm{Ni}_{3} \mathrm{Al}$ (111) peak is cannot be identified in the MCB only XRD spectrum. Thus, MCB at 4000 rpm for 1 hour is not sufficient to produce ODS Ni-based alloys. 


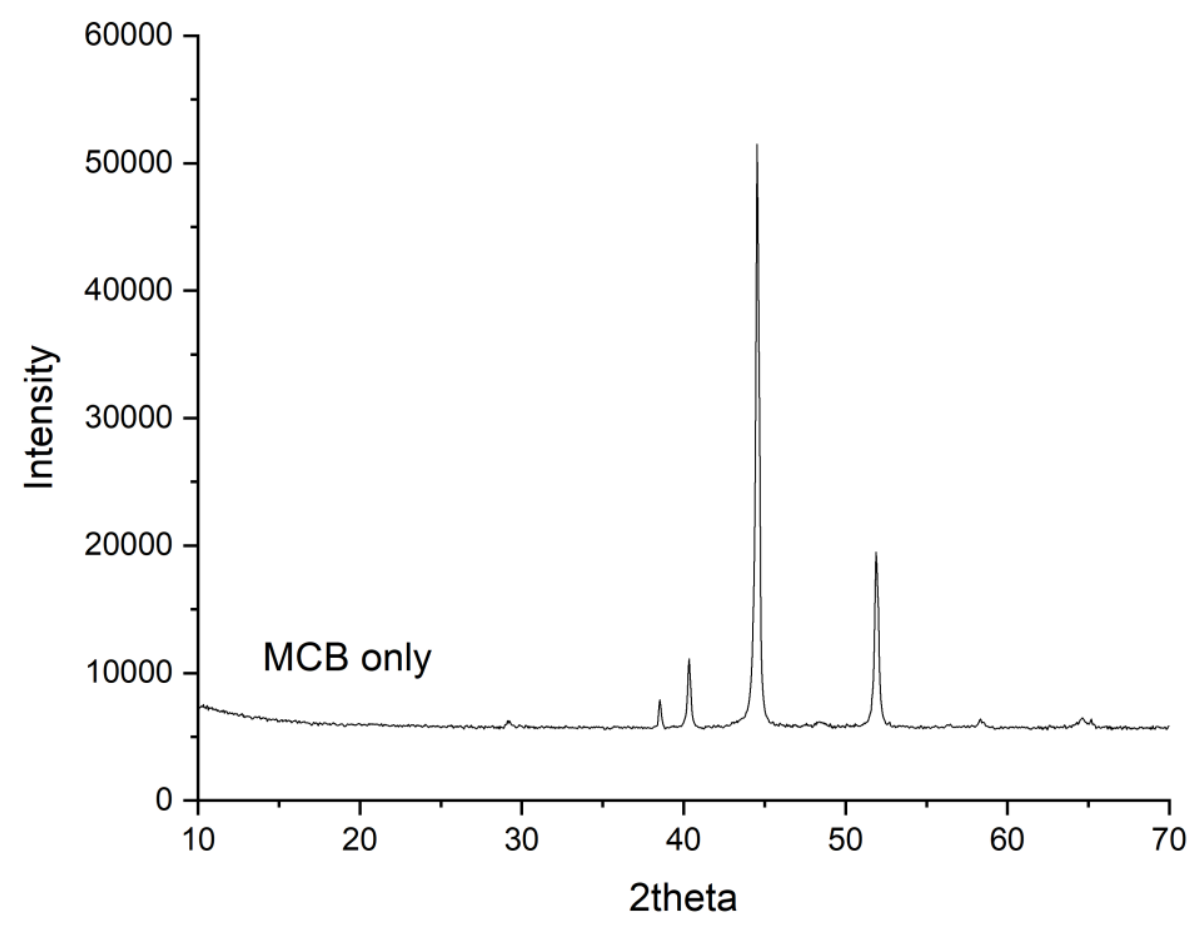

Figure 5-11. XRD spectrum of MCB-only processed powders

Given the XRD spectrum of MCB-only does not show evidence of the powders reach steady state and the lack of $\mathrm{Ni}_{3} \mathrm{Al}$ (111) peak, further MA via planetary ball milling is done. Several parameters of the BM process influence the microstructure of the powders. Given stainless steel milling containers, stainless steel grinding medium, and stearic acid $0.5 \mathrm{wt}$. \% of the powder, the milling speed, milling time, and BPR will be optimized. Figure 5-11 shows the XRD spectrum of MCB+BM powders with powders subjected to milling speed of $300 \mathrm{rpm}$. It is observed from the three spectrums, NiAl (110) and $\mathrm{Ni}_{3} \mathrm{Al}(110)$ peaks appear at $44^{\circ}$ and $52^{\circ}$, respectively. The individual elemental peaks shown in Figure 5-9 are not observed, with the exception of W. The W peak (angle of $40^{\circ}$ ) undergoes broadening, suggesting a change in the crystallographic structure because of formation of solid solutions during the BM process. Because the BM process undergoes severe plastic deformation, broadening of all the major peak are shown. Thus, the combined MCB+BM process allows for uniform dispersion of $\mathrm{Y}_{2} \mathrm{O}_{3}$ and generation of a finer crystallographic structure. 


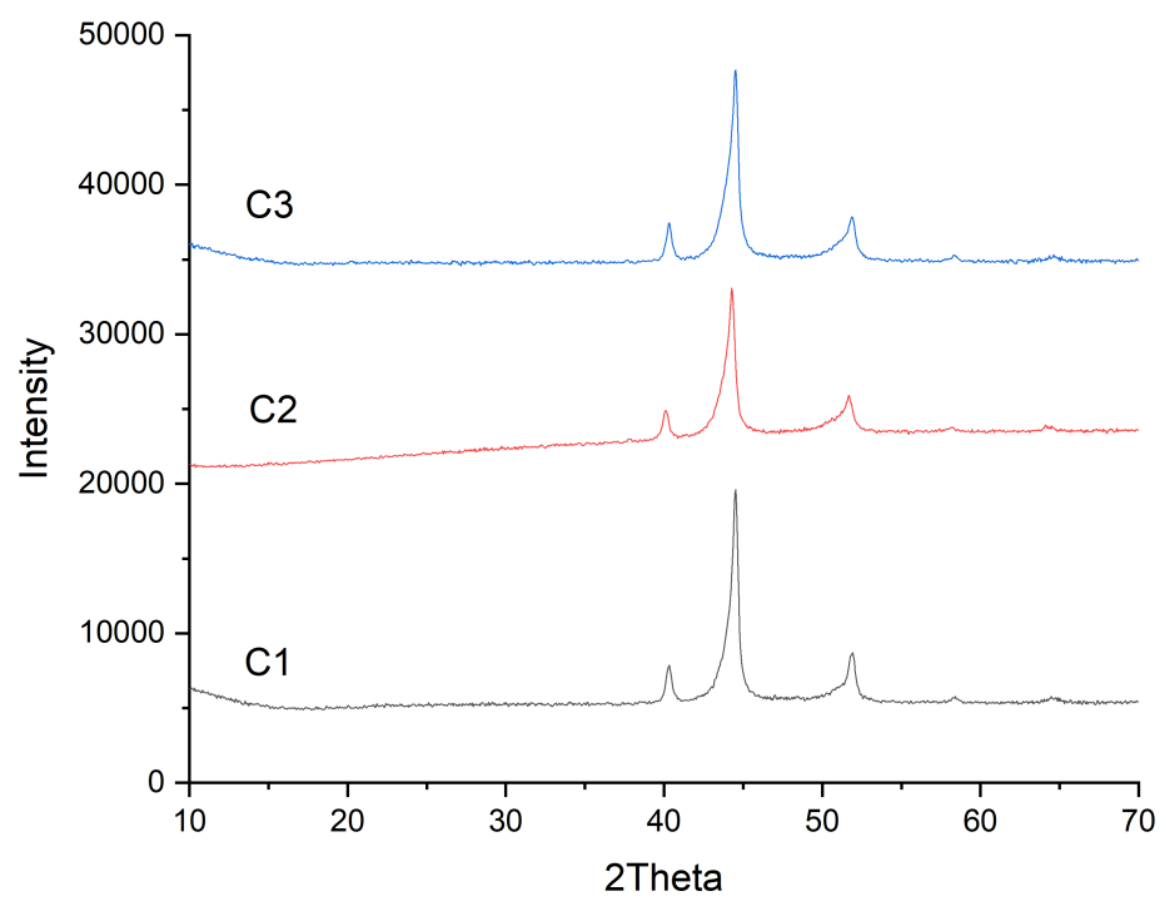

Figure 5-12.XRD spectrum of MCB+BM process at $300 \mathrm{rpm}$ (C1) BPR 15:1 and 15 hours (C2) BPR 30:1 and 15 hours (C3) BPR 30:1 and 45 hours.

With a fixed milling speed of $300 \mathrm{rpm}$ and milling time of 15 hours, an increase of BPR from 15:1 to 30:1 shows a further shift in Ni peak $\left(58^{\circ}\right)$. The double peak shared by $\mathrm{Ni}$ and $\mathrm{Ni}_{3} \mathrm{Al}$ (111) appears to "round". In other words, the intermetallic precipitate, $\mathrm{Ni}_{3} \mathrm{Al}$, becomes prevalent with increased BPR and all other parameters remaining constant. The $\mathrm{NiAl}$ (110) peak also experiences broadening, showing a further refined crystallographic structure. Ni lattice peaks appear at slightly lower Bragg angle and Al lattice peaks at slightly higher Bragg angle with an increase in milling time and BPR at fixed rotation speed of $300 \mathrm{rpm}$. The shifting of the Ni lattice peaks and eventual disappearance of the $\mathrm{Al}$ lattice peak indicates the formation of $\mathrm{y}-\mathrm{NiAl}$ solid solution $[24,41,42]$. Also, the existence $Y^{\prime}-\mathrm{Ni}_{3} \mathrm{Al}$ intermetallic compound is shown after 15 hours of milling time and a BPR of 15:1. The double peaks in which the higher angle peak corresponds to the Ni-lattice peak.

However, a constant BPR of 30:1 and an increase of milling time from 15 hours to 45 hours does not show much difference in the microstructure of the powders. The $\mathrm{Ni}_{3} \mathrm{Al}$ peak still shows evidence of Ni particles. Therefore, increasing milling time with a large BPR of 30:1 did not result in the powders to reach steady state. Further increase of the BPR will lead to lower powder yield, while increasing milling time is not practical for fabrication of ODS Ni-based powders. Thus, increasing the milling speed is the next processing parameter to adjust. 


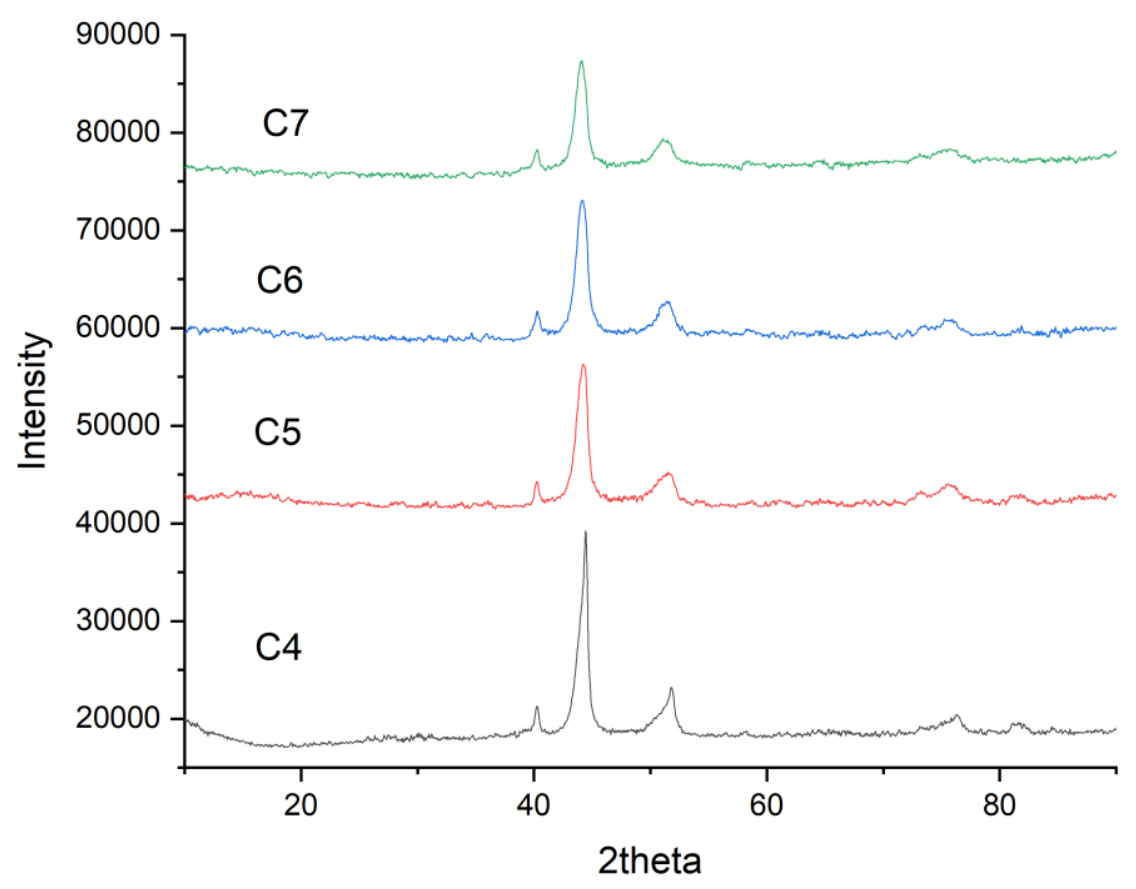

Figure 5-13. XRD spectrum of MCB+BM process at BM milling speed of $400 \mathrm{rpm}$ and BPR of 15:1 (C4) 15 hours (C5) 25 hours (C6) 30 hours (C7) 40 hours.

Increasing the rotation speed to $400 \mathrm{rpm}$ and a constant BPR of 15:1 shows an increased displacement of the Ni peaks to lower angles. Further broadening of the NiAl and $\mathrm{W}$ lattice peaks is also evident in the XRD spectrum. This not only suggests a decrease in grain size of the ODS particles, but an increase in concentration of $\mathrm{Ni}_{3} \mathrm{Al}$ precipitates. Milling time of 15 hours to 25 hours shows the double peak to shift more towards a single peak. The further shift of the Ni peak shows an increase in concentration of the $\mathrm{Ni}_{3} \mathrm{Al}$ (111), unlike the BM configuration of milling speed $300 \mathrm{rpm}$. At milling speed of 30 hours, the peak is now a single peak with no further existence of $\mathrm{Ni}$.

Based on the XRD spectrums, milling speed influenced the microstructure of the ODS Ni-based ODS alloys more than the milling time and BPR. With the higher milling speed, a lower BPR of 15:1 allows for higher powder yield. The steady state is also seen to appear after 30 hours of the BM process.

\subsection{Analysis of As-Printed Sample}

The MCB+BM processed powders were applied on LESN 450 system with a nominal laser spot size of $240 \mu \mathrm{m}$ and a MAR-M247A flat plate was selected as the substrate. Three as-printed samples were AM produced with a laser travel speed of 33 $\mathrm{mm} / \mathrm{s}$. The laser power was selected as $200 \mathrm{~W}, 250 \mathrm{~W}$ and $270 \mathrm{~W}$, producing linear energydefined as laser power $(\mathrm{J}) / \mathrm{beam}$ travel speed $(\mathrm{mm})$ [43]. Therefore, the linear energy is $6.06 \mathrm{~J} / \mathrm{mm}, 7.75 \mathrm{~J} / \mathrm{mm}$ and $8.33 \mathrm{~J} / \mathrm{mm}$ respectively. Hatching lines were made at 0 。 horizontally in the first layer and then were rotated $90^{\circ}$ for the next layer. The hatch 
spacing was $0.1524 \mathrm{~mm}$, the layer thickness was $0.1 \mathrm{~mm}$ and the powder feeding rate was $8 \mathrm{rpm}$.
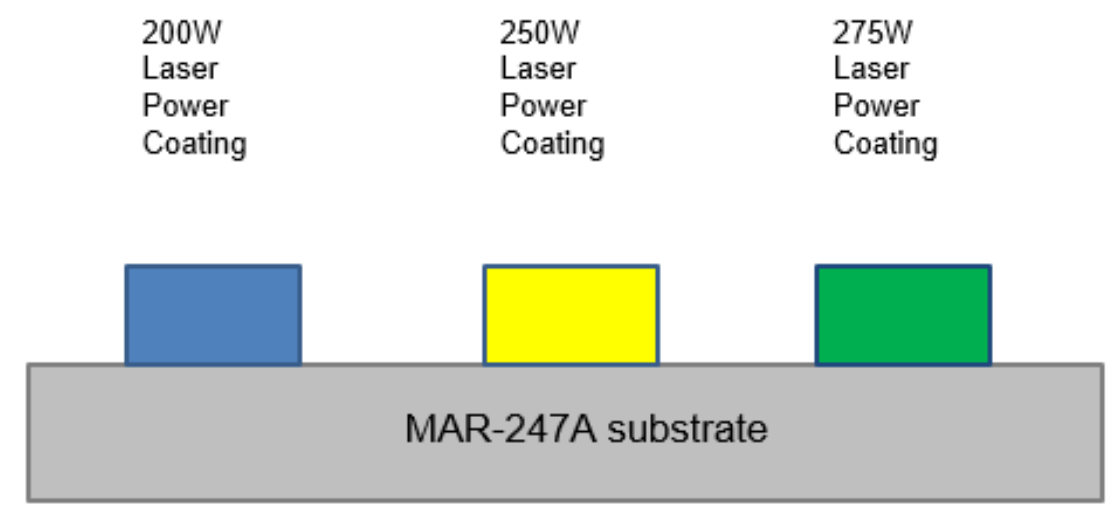

Figure 5-14. A schematic of the first batch of LENS as-printed samples[44].

Table 5-1. The experimental density relative density, and input linear energy of asprinted samples

\begin{tabular}{cccc}
\hline $\begin{array}{c}\text { As-printed } \\
\text { samples }\end{array}$ & $\begin{array}{c}\text { Experimental Density } \\
\left(\mathrm{g} / \mathrm{cm}^{3}\right)\end{array}$ & $\begin{array}{c}\text { Relative } \\
\text { Density } \\
(. \%)\end{array}$ & $\begin{array}{c}\text { Input Linear } \\
\text { Energy } \\
(\mathrm{J} / \mathrm{mm})\end{array}$ \\
$200 \mathrm{~W}$ & 5.67 & $66.63 \%$ & 6.06 \\
$250 \mathrm{~W}$ & 6.33 & $74.38 \%$ & 7.75 \\
$275 \mathrm{~W}$ & 7.13 & $83.78 \%$ & 8.33 \\
\hline
\end{tabular}

Table 7 shows the experimental density and relative density of as-printed samples. The as-printed density of samples increased with the increase of the linear energy, meaning the more linear energy, the denser microstructure and the more chance of forming a melt pool. Figure 5-15 shows the SEM images of the cross-section of 200W$6.06 \mathrm{~J} / \mathrm{mm}, 250 \mathrm{~W}-7.75 \mathrm{~J} / \mathrm{mm}$, and $275 \mathrm{~W}-8.33 \mathrm{~J} / \mathrm{mm}$ as-printed samples. The influence of the laser linear energy on the microstructure of as-printed parts can be observed at low magnification.

The SEM images show evidence of pores existing in the as-printed ODS part showing the cause of the low density of the as-printed sample. Columnar structures are shown in Figures 15 (b) and (c), but not in (a), which suggests higher input linear energy would cause columnar structure because there is more thermal driving force for grain growth in the microstructure. However, there is no clear evidence of pores in a low magnification in Figure 5-15 (b) and (c). The growth directions of columnar structure and the building direction of the as-printed ODS parts are displayed by arrows in the SEM image. The growth direction of the columnar structure was found that it was approximately $45^{\circ}$ from the building direction. 

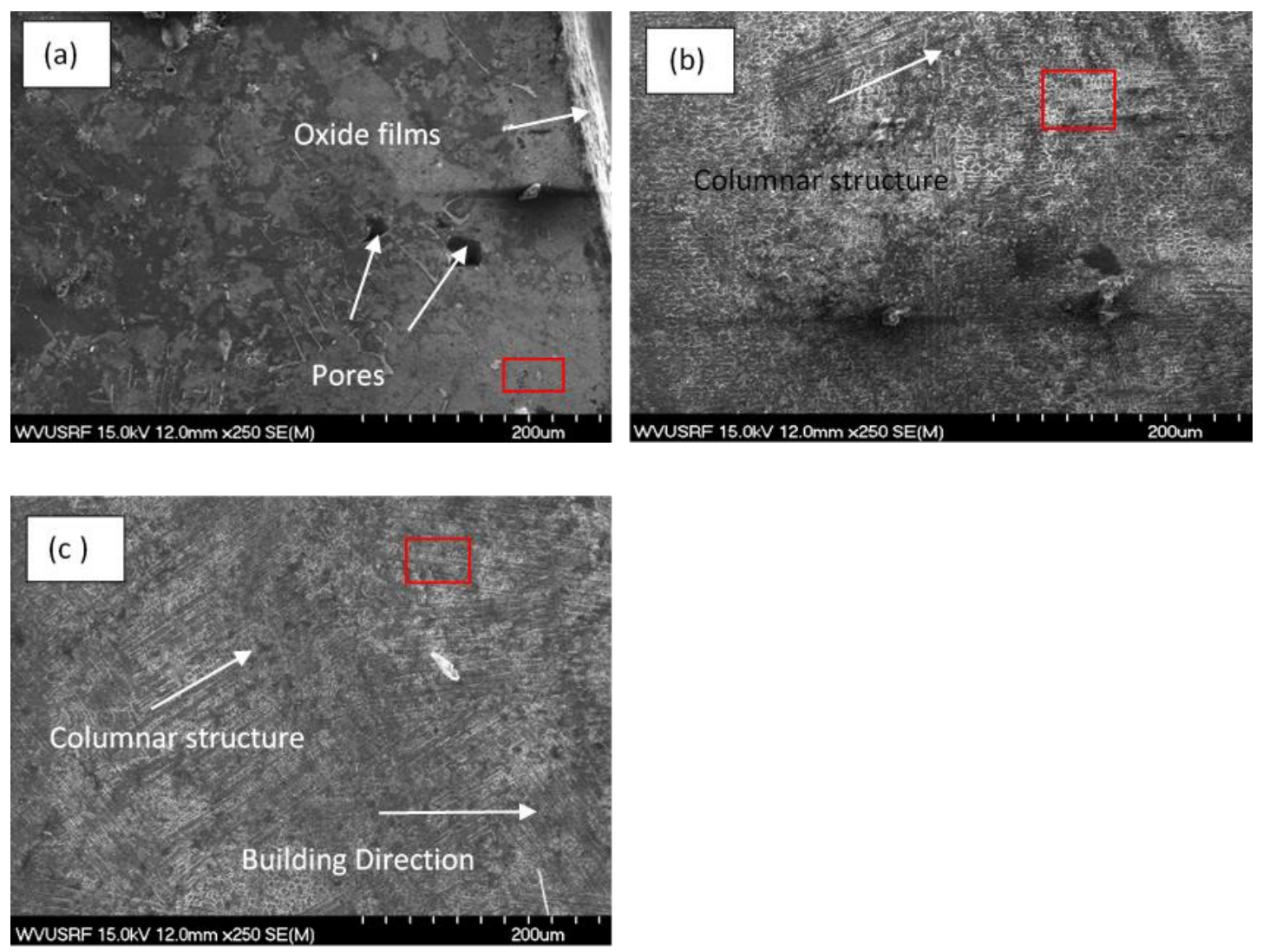

Figure 5-15. SEM showing images of the characteristic microstructures of as-printed ODS parts at different laser power and input linear energy: (a). 200W-6.06 J/mm; (b). $250 \mathrm{~W}-7.75 \mathrm{~J} / \mathrm{mm}$; (c). $275 \mathrm{~W}-8.33 \mathrm{~J} / \mathrm{mm}$

As the laser power increased, the columnar structure arrays became finer, exhibiting a typical epitaxial growth along the growth direction. However, in Figure 5-15 (b), as the building direction moved forward, the columnar structure became dispersed and unclear. On the other hand, the columnar structure in Figure 5-15 (c) is still evident as the building direction moved forward. Thus, the input energy of $250 \mathrm{~W}-7.55 \mathrm{~J} / \mathrm{mm}$ sample was not sufficient and decreased with advancement of the building direction comparing the $275 \mathrm{~W}-8.33 \mathrm{~J} / \mathrm{mm}$ sample [44].

Figure 5-16 shows the characteristic microstructures of as-printed ODS parts in the specific locations (as indicated in the red block in Figure 5-15) at higher magnification. No columnar structures were found in Figure 5-16 (a), but tiny upright $\gamma^{\prime}$ phase was observed. It was hard to identify every columnar dendrite in Figure 5-16 (b) since severe clustering occurred during the solidification process within the melt pool. Also, short cracks were found in the inter-dendritic region in the SEM image. The continuous columnar dendrites are clearer in Figure 5-16 (c), but small and short cracks were still 

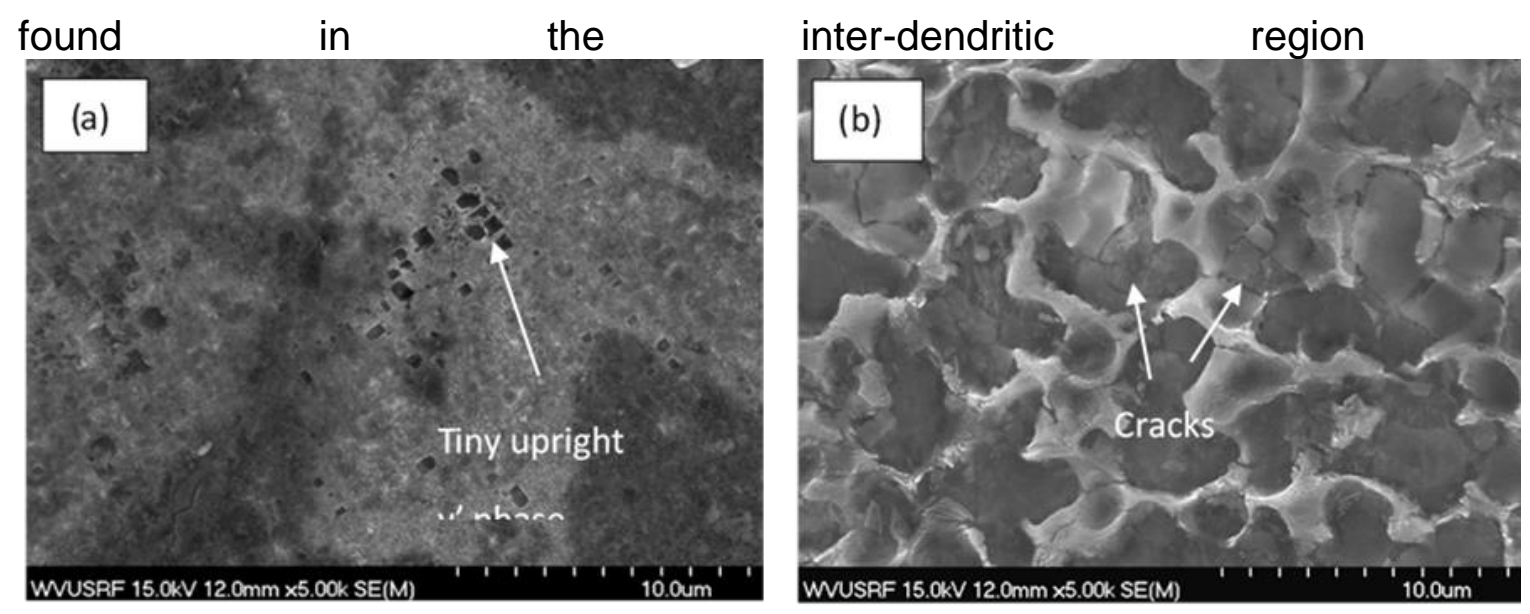

[44].

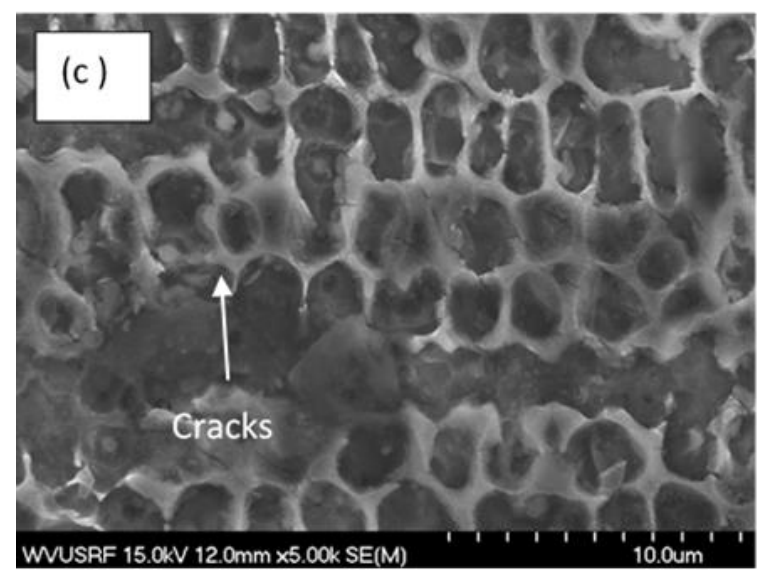

Figure 5-16. High-magnification of SEM micrographs showing the characteristic microstructures of as-printed ODS parts in: (a). Figure 5-19 (a); (b). Figure 5-19 (b); (c). Figure 5-19 (c).

Unlike the ambiguous epitaxial characteristics shown in Figure 5-16 (b), the columnar dendrites generally displayed the epitaxial growth with a higher degree of clustering on increasing laser power. Generally, the major input energy and heat induced by laser radiation were transmitted through the substrate of previous as-printed layers, which in turn facilitated the formation of typical columnar dendrite structures along the building direction $[43,45,46]$. Also, residual thermal stress as a result of the repeated rapid melting and solidification at a higher laser power was remained in the workpiece [47] which results in the generation of thermal cracks in both dendrite and inter-dendritic regions that were observed in the SEM micrographs [44].

Increasing laser power caused the instantaneous and overall temperature within the melt pool to increase substantially because more heat and energy induced by the laser was generated during the process. Therefore, the heat accumulated during the process allowing the internal energy and thermodynamic potentials to improve. The improvement enhanced the epitaxial growth of the columnar dendrites and the dispersion performance [46]. 
In order to examine the chemical composition of the columnar dendrites and the inter-dendrite zones that were observed in different process conditions. EDS point scan was conducted to identify the chemical composition as shown in Figure 5-17. For EDS spot $1 \mathrm{C}, \mathrm{O}, \mathrm{Cr}, \mathrm{Al}, \mathrm{Ni}$, and $\mathrm{Y}$ are present and the major component is $\mathrm{Cr}, \mathrm{Al}$, and $\mathrm{O}$. Thus, formation of complex oxides and carbide occur during the process. The $\mathrm{Y}_{2} \mathrm{O}_{3}$ tended to grow on the grain boundary. EDS spots show $\mathrm{C}, \mathrm{O}, \mathrm{Ni}, \mathrm{Cr}, \mathrm{Al}$, and $\mathrm{W}$. EDS point analysis indicated that carbon was introduced during the process. Because the powders were processed by $\mathrm{MCB}+\mathrm{BM}$ process, the PCA, stearic acid, with the composition of $\mathrm{CH}_{3}$ $\left(\mathrm{CH}_{2}\right)_{16} \mathrm{CO}_{2} \mathrm{H}$ to boost the MA effect during the BM process. Some of the carbon remained after the BM process and reacted with major elements $\mathrm{Ni}, \mathrm{Cr}$, and $\mathrm{Al}$ to form carbides during the printing process [44].
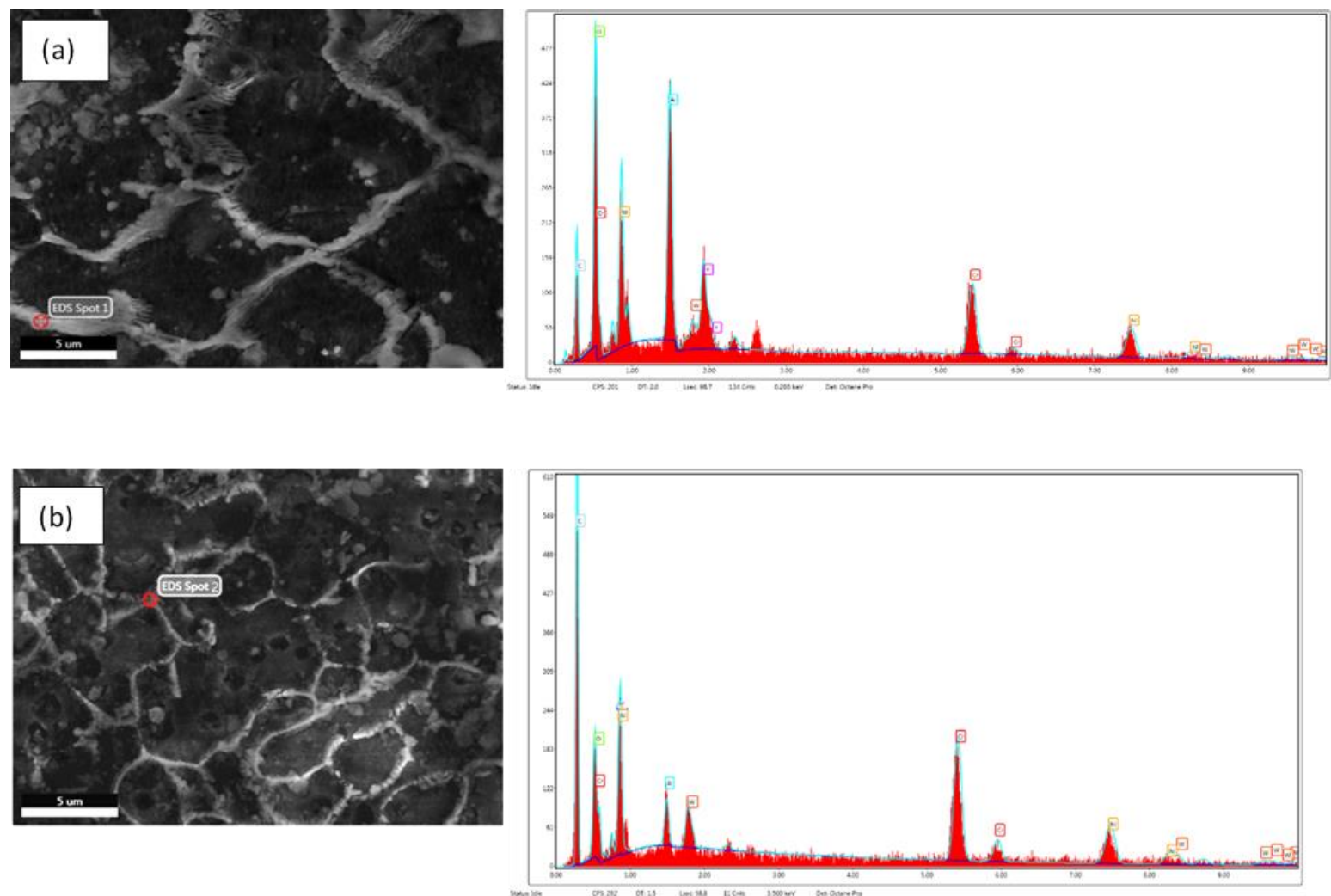

Figure 5-17. EDS point analysis of as-printed ODS parts at different laser powder and input linear energy: (a). $250 \mathrm{~W}-7.75 \mathrm{~J} / \mathrm{mm}$ in mid-layer; (b). $275 \mathrm{~W}-8.33 \mathrm{~J} / \mathrm{mm}$ in mid-layer. 


\section{CONCLUSIONS}

\subsection{Powder Characterization}

In this study, a novel MCB+BM technique was used to fabricate Ni-based ODS alloy powders with nanocrystalline microstructure and uniform dispersion of $\mathrm{Y}_{2} \mathrm{O}_{3}$. The powders were initially processed via MCB for 1 hour at $4000 \mathrm{rpm}$ and then followed by planetary BM process for over 10 hours at $400 \mathrm{rpm}$. The ODS particles microstructure were characterized by XRD and the size, size distribution, and morphology by SEM and particle analyzer.

The $\mathrm{Y}_{2} \mathrm{O}_{3}$ nanoparticles were shown to be bonded and coated on the master particles ( $\mathrm{Ni}$ and $\mathrm{Cr}$ ) and form a nano-size amorphous film during the MCB process. Evidence of the $\mathrm{Y}$-NiAl matrix is shown in the XRD spectrum of MCB only; implying the elemental Al powders fractured and bonded onto the Ni powders due to large shear force during the process.

Further MA processing via planetary ball mill introduced the intermetallic precipitates $\mathrm{Y}^{\prime}-\mathrm{Ni}_{3} \mathrm{Al}$ and nanocrystalline microstructure. Constant milling speed and increase in BPR introduced higher impact energy, promoting further cold-welding and the powders, thus providing larger concentration of $Y^{\prime}$ precipitates. Increase of milling time with constant BPR show little to no difference in the microstructure of the powders, however an increase in milling speed allow for higher powder yield and desirable microstructure.

Milling speed of $400 \mathrm{rpm}$ shows powders are more spherical in shape (minimal individual particles appear "flaky"). Fixed milling speed of $400 \mathrm{rpm}$ and increase milling time show powders with uniform particle size distribution and average particle size of 10 $\mu \mathrm{m}$ which are suitable for AM application.

\subsection{AM processing of MCB+BM powders}

MCB+BM processed powders were applied on LENS 450 system and the asprinted samples were characterized by SEM/EDS. The results suggest the higher the input linear energy, the denser the material will be. Also, an increase in input energy shows an increase in the $y^{\prime}$ precipitation of the microstructure. Overall, laser power is shown to generate a higher melt pool temperature, thus providing sufficient formation of Y' phase. 


\section{RECOMMENDATIONS FOR FUTURE WORK}

Although it is determined a milling speed of $400 \mathrm{rpm}$, BPR of 15:1, and milling time of 40 hours was the optimal BM procedure, further investigation of other BM processing parameters can allow for shorter milling time. Also, particles shown were not perfectly spherical and research of PCA or development of a new MA device can aid in production of perfect spherical particles.

FEA simulations of the micro-indentation technique did not agree with the experimental results. Further simulation on spherical parts with irregular shape can be done to determine a shape factor. Derivation of the "true" loading condition during the indentation testing can also be investigated. FEA simulation of the multiple loading-partial unloading micro-indentation technique gives confidence the technique can be used to determine mechanical properties of powders. Experimental results on spherical balls of known material properties were done. Results were not accurate, however, it is believed misalignment of the load mechanism, surface roughness of the flat indenter, and surface finish/shape influence results. Preliminary review, theory, and results are given in the Appendix. 


\section{A. Appendix}

Since the microstructure of the ODS powder will influence the mechanical properties of AM-processed structural parts, implementation of a load-based multiple loading-partial unloading micro-indentation technique will be used to evaluate the mechanical properties of metallic powders. Numerical simulation was carried out on spherical and non-spherical particles in order to determine a shape factor function for non-spherical particles.

\section{A.1 Mechanical Properties of powders/particles}

Indentation is a useful mechanical testing process that uses hard, geometrically specific materials as an indenter to submerge into a test material. The test material's reaction to the applied load of the indenter depends on the mechanical properties. The depth and surface area provide enough information to determine the mechanical properties. Various techniques can be used to determine the indentation depth and area, such as depth sensing and optical technology. Although indentation testing to obtain material mechanical properties was first developed for alloys, the technique has become helpful in a number of industrial sectors. Since the 1800's indentation testing was first used for hardness testing due to the technique's ability to perform non-destructive evaluation (NDE). Unlike conventional methods to determine mechanical properties of materials, indentation has shown the capability to determine properties of materials with ambiguous geometry or sensitive in nature i.e., thin films, coatings, and nano-systems. The following are examples of indentation techniques used to determine mechanical properties of particles.

\section{A.2 Contact Mechanics and Indentation}

A general case of contact stress occurs when two contacting bodies loaded against each other have perpendicular principle curvatures at the contact point. A specific case will be two spheres in contact (which includes a sphere on a flat plane). Hertz determined the radius of the contact area for two solid spheres with diameter $d_{1}$ and $d_{2}$ can be represented as [48]:

$$
\begin{gathered}
a=\sqrt[3]{\frac{3 P\left[\left(\frac{1-v_{1}^{2}}{E_{1}}\right)+\left(\frac{1-v_{2}^{2}}{E_{2}}\right)\right]}{8\left(\frac{1}{d_{1}}+\frac{1}{d_{2}}\right)}} \\
\frac{1}{E_{R}}=\frac{1-v_{1}^{2}}{E_{1}}+\frac{1-v_{2}^{2}}{E_{2}}
\end{gathered}
$$


Where $E_{R}$ is the reduced Modulus of Elasticity, $E_{1,2}$ are the moduli of elasticity for spheres 1 and 2, and $\mathrm{V}_{1}, 2$ are the Poisson's ratio for spheres 1 and 2, respectively. Allowing the diameter of the seconds sphere to approach infinity and assuming $a^{2}=R h_{e}$ (where $R$ is the radius of the spherical indenter and $h_{e}$ is the indentation depth), Equations A.6 and A.7 evaluate to:

$$
P=\frac{4}{3} E_{R} R^{\frac{1}{2}} h_{e}^{\frac{3}{2}}
$$

Young's modulus measurement from materials testing can be determined from the unloading data of a loading-unloading indentation test and Equation A.8 can be re-written as:

$$
\frac{d P}{d h}=2 E_{R} \sqrt{\frac{A}{\pi}}
$$

Where $A$ is the projected area of the indentation and a function of indentation depth. However, measurement of the projected area or indentation depth requires precise measurements. As a result, an alternative method, developed at West Virginia University, allows for a theoretical solution for the Young's Modulus measurement. Equation A.9 can be rewritten as [49]:

$$
\frac{d h}{d P}=C P^{-\frac{1}{3}}
$$

Where $C=\left(6 \mathrm{E}_{R}{ }^{2} \mathrm{R}\right)^{-1 / 3}$ and related the reduced Young's modulus of the system. Thus, only the unloading stiffness and load are required to determine the Young's modulus.

Developed at West Virginia University, a multiple loading partial unloading indentation technique is shown to determine the mechanical property of materials nondestructively. Figure A-1, shown below, show a simple schematic of the indentation system. As seen, the system has a load cell to record the reaction force, piezoelectric (PZT) actuator to implement indentation, and a spherical indenter. 


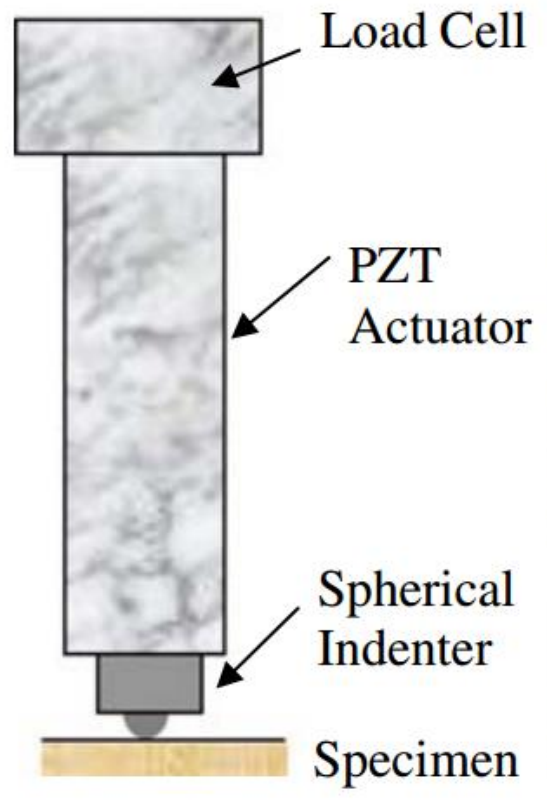

(a)

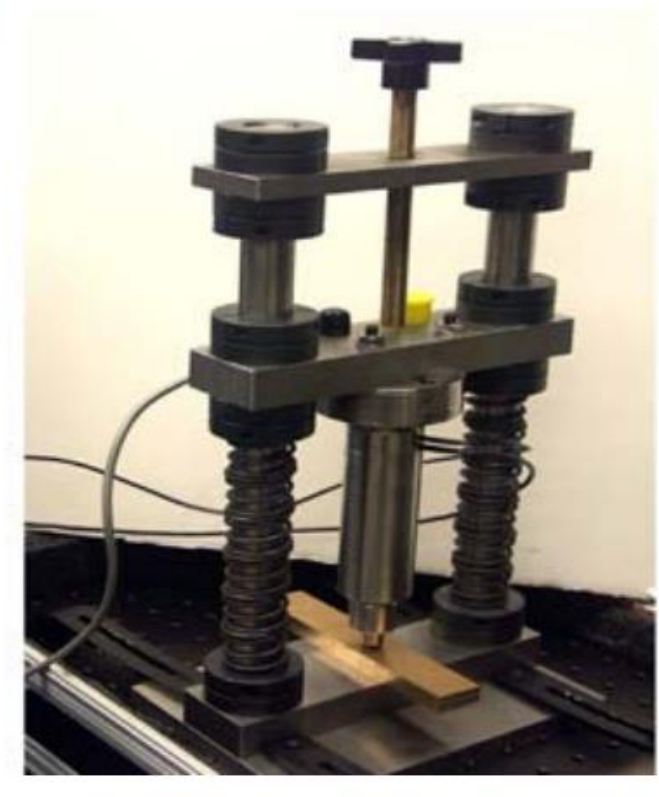

(b)

Figure A-1. Table-top load depth sensing indentation system (a) Schematic of indentation system and (b) indentation system [49].

Feng et al., shows the loading apparatus and sample material is subjected to the indentation load caused by the PZT. As a result, the total indentation includes the indentation depth and material deformation. Thus, the total indentation $\left(\mathrm{h}_{\text {total }}\right)$ is:

$$
h_{\text {total }}=h+h_{s}
$$

Where $\mathrm{h}$ is the indentation depth and $\mathrm{h}_{s}$ is the deformation of the indention system. Taking the derivative with respect to the applied load and substituting Equation A.10, you will find:

$$
\frac{d h_{\text {total }}}{d P}=C P^{-\frac{1}{3}}+C_{s}
$$

Where $\mathrm{C}_{\mathrm{s}}$ is the system compliance. It was shown the slope $\mathrm{C}$ can be used to determine the reduced modulus of the system. Thus, if the system compliance can be measured or calibrated during the indentation, evaluation of the indentation to the material does not need evaluation [49].

Unfortunately, the compliance is not constant depending on the sample size and other factors. As a result, the multiple loading-partial unloading approach allows one to determine the slope as long as the system compliance does not change during the indentation test. Figure A-2 shows the load-displacement curve with multiple partial unloading for a single indentation test as well as the linear relationship. 


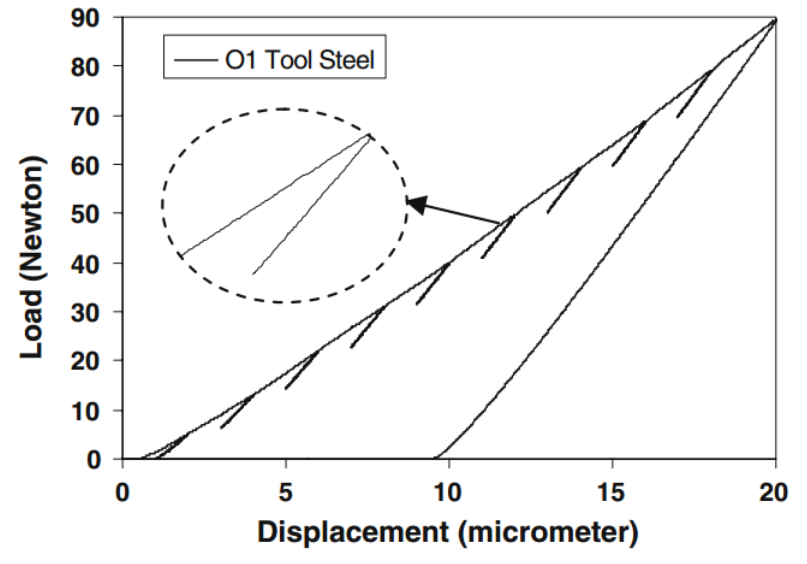

(a)

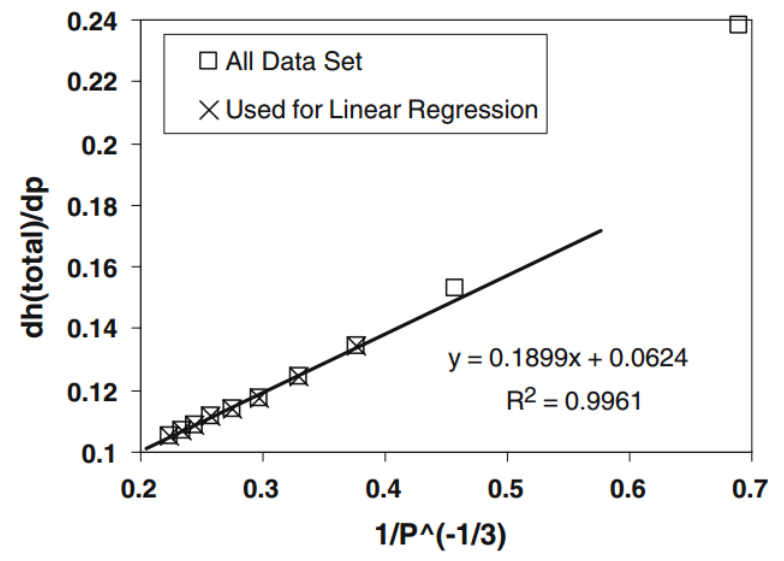

(b)

Figure A-2. Experimental results from multiple loading partial unloading on $\mathrm{O} 1$ tool steel (a) Experimental load-displacement curve and (b) linear relationship[49].

\section{A.3 Mechanical Property Analysis}

The multiple loading-partial unloading technique developed at West Virginia University is shown to determine the mechanical properties of a flat coupon sample with use of a spherical indenter. The adjustment of the BM parameters was done, not only to achieve a nanocrystalline microstructure and uniform dispersion of $\mathrm{Y} 2 \mathrm{O} 3$, but also to produce powders suitable for AM processing. Uniform size distribution, average particle size greater than $10 \mu \mathrm{m}$, and spherical morphology was also an objective. It is shown the Ni-based ODS powders produced by BM parameters of $400 \mathrm{rpm}, 15: 1 \mathrm{BPR}$, and 40 hour milling time have near spherical shape. Thus, it is feasible the indentation technique can be used to determine the mechanical properties of the powders, given the indenter is now flat and the sample spherical. Finite Element Analysis (FEA) was done to determine the possibility of this assumption.

\section{A.3.1 FEA Simulation of Spheres}

Both the flat indenter and spherical specimen are treated as bodies of revolution to avoid practical issues of the three dimensional nature of the problem. Therefore, a three dimensional analysis must be done for accurate results. Simulia ABAQUS CAE software was used to for the following simulations. Because the indenter and spherical sample will behave as three dimensional bodies under axially symmetric loading conditions, an axisymmetric simulation will be done and the applied load will be considered the symmetry axis. The indenter and specimen are shown in Figure A-3, as well as the boundary conditions and load. 


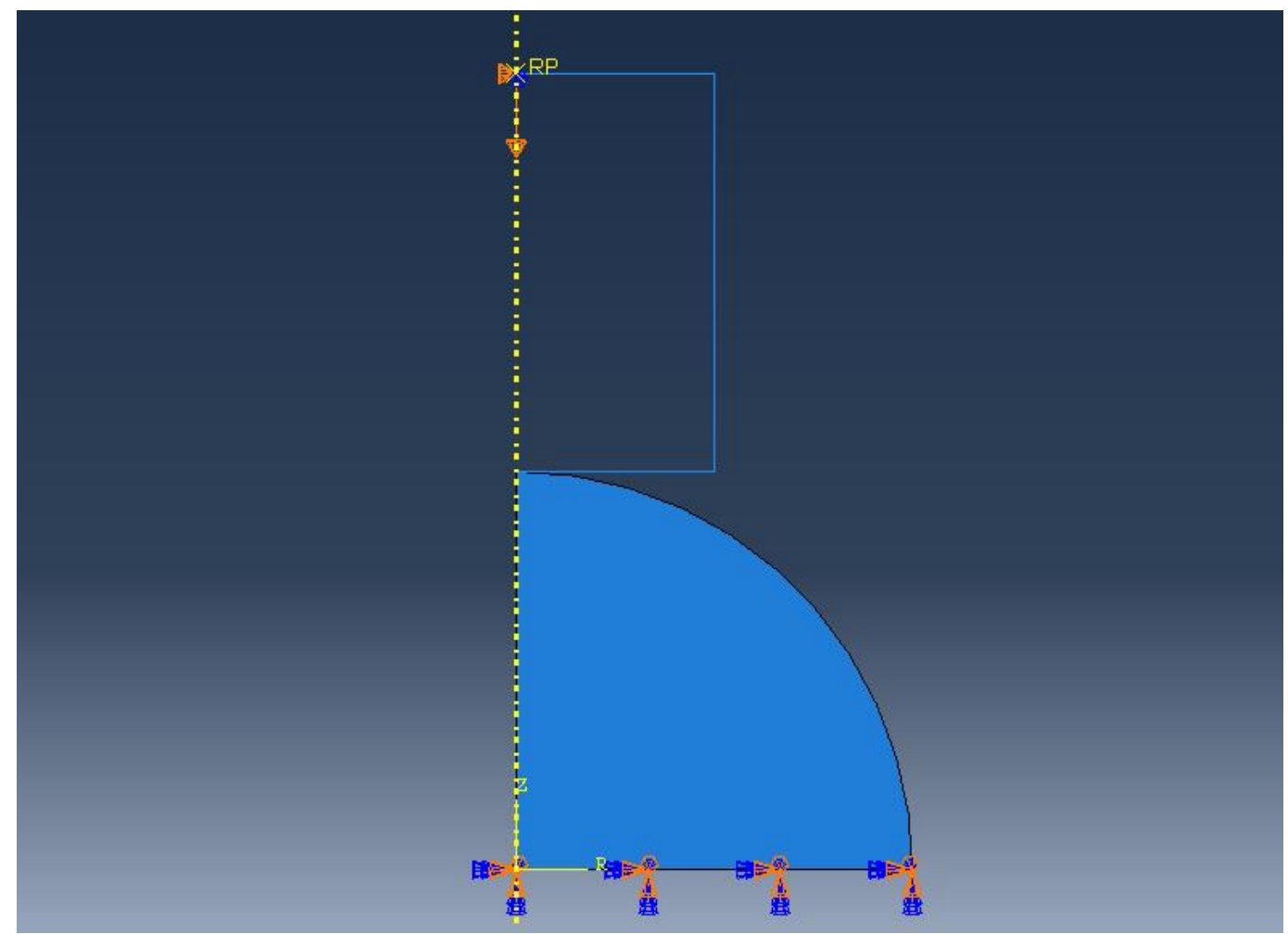

Figure A-3. Schematic of spherical specimen under the flat indenter with boundary conditions shown.

During indentation, the indenter chosen for testing has higher hardness and stiffness than the sample. As a result, an analytical rigid body is used to model the indenter. The indenter will load the sample $3 \mu \mathrm{m}$ and unload $1 \mu \mathrm{m}$ to $30 \mu \mathrm{m}$ (for a total of 10 steps). This allows for 10 points to determine the slope and eventually reduced modulus given Equation 3.12. The loading of the indenter onto the sphere is along the symmetry line. A boundary condition will be on the flat of the sphere and acts as rollers to prevent movement in the $\mathrm{x}$-direction. The center of the sphere has a fixed condition to prevent displacement in the $y$-direction. Hard contact between the rigid body and deformable sphere is assume with friction coefficient of 0.5 and small sliding

Because very small indentations are being simulated, meshes near the indenter must be fine to describe the deformation and stress gradients for accuracy [50]. Therefore, a fine mesh of $50 \mu \mathrm{m}$ was used near the point of contact and gradually increased to $400 \mu \mathrm{m}$ farther from contact. A 3-node linear axisymmetric triangle (CAX3) mesh was used for the particle. Figure A-4 shows the mesh of the particle. Due to the changing mesh, when two elements are connected in the distribution, the middle node on the common face is constrained to lie on a straight line. 

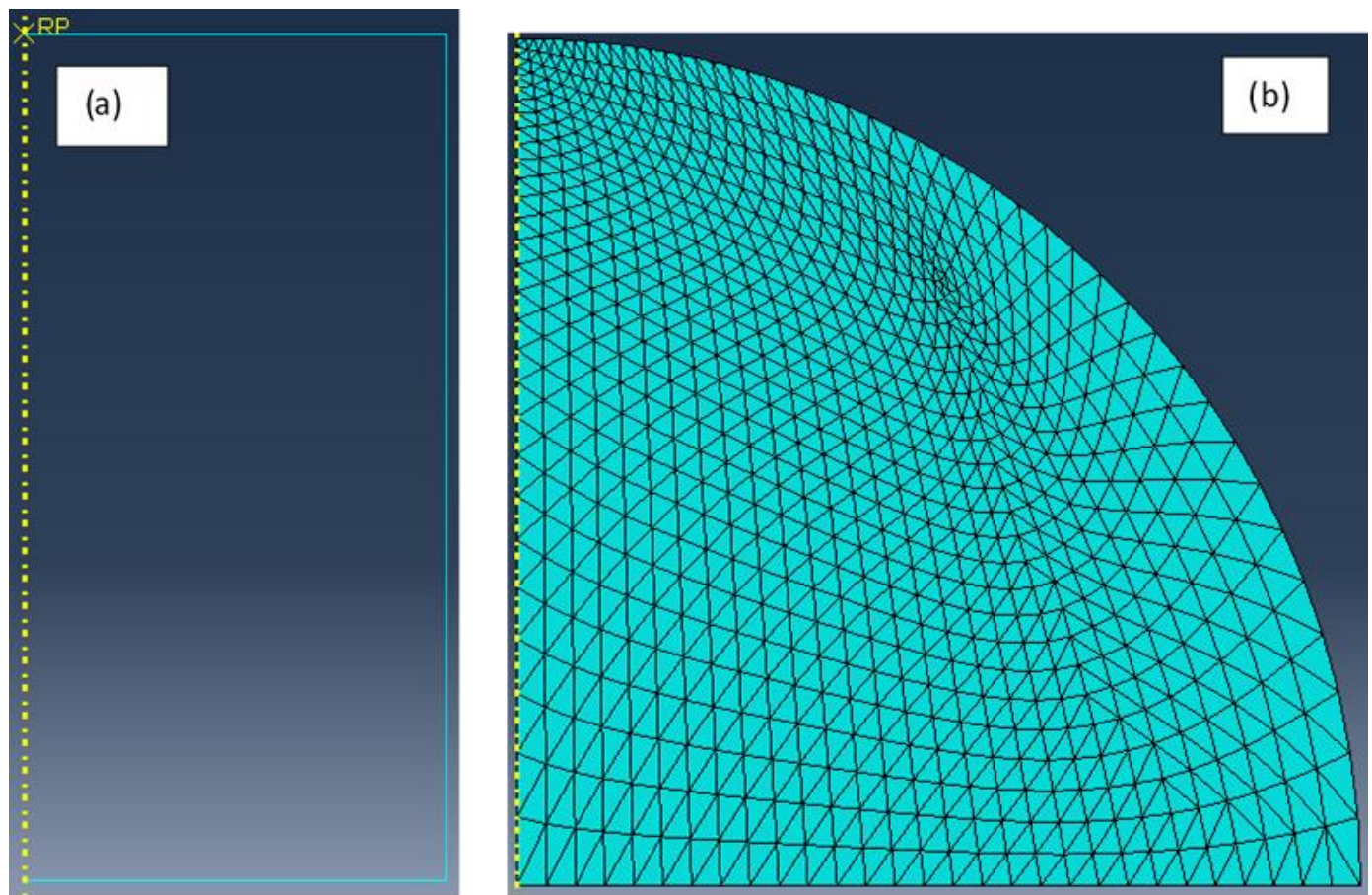

Figure A-4. Mesh distribution of (a) analytical rigid indenter and (b) deformable sphere

The flat analytical rigid indenter is considered to have infinite stiffness (as stated earlier) and the spherical particle is chosen to be Stainless Steel 316. Figure A-5 shows the stress strain curve of common SS 316 at different temperatures. In this study, room temperature lot 2 will be used as the deformable part material property. The Young's Modulus of the material is known to be $193 \mathrm{GPa}$ and the hardening coefficient is determined from power law and Figure 36. 


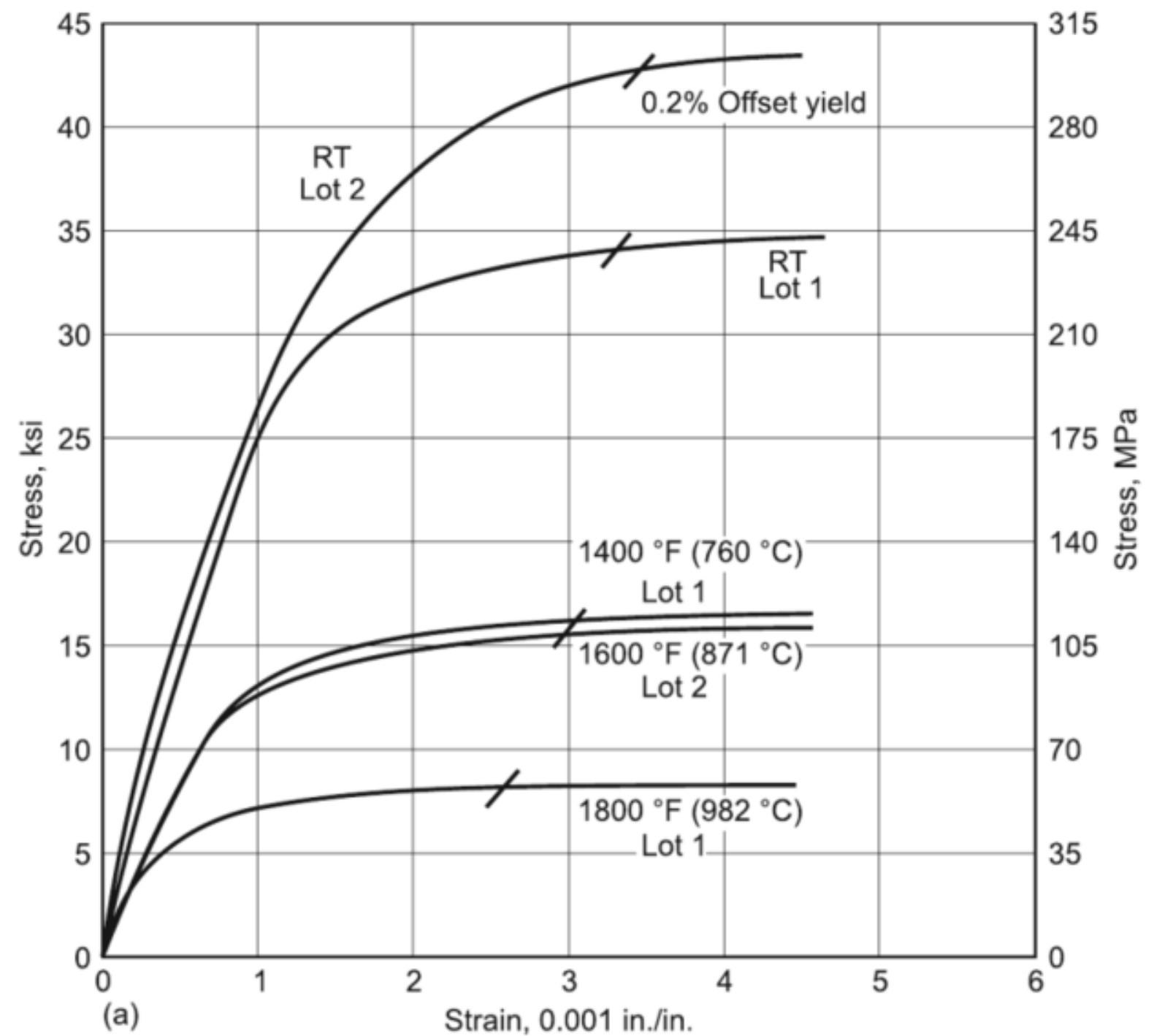

Figure A-5. Stress Strain curve of Stainless Steel 316 at room temperature, $1400^{\circ} \mathrm{F}$, $1600^{\circ} \mathrm{F}$, and $1800^{\circ} \mathrm{F}[51]$.

Simulation using the multiple loading partial unloading technique was done on spherical deformable parts of size, $10 \mathrm{~mm}, 5 \mathrm{~mm}, 3 \mathrm{~mm}, 2 \mathrm{~mm}, 1 \mathrm{~mm}$, and $0.5 \mathrm{~mm}$ to determine the stiffness response. Figures A-6 $-\mathrm{A}-11$ show the respective $\frac{d h}{d P} v s . P^{-\frac{1}{3}}$ plots, linear regression line, and correlation coefficient. 


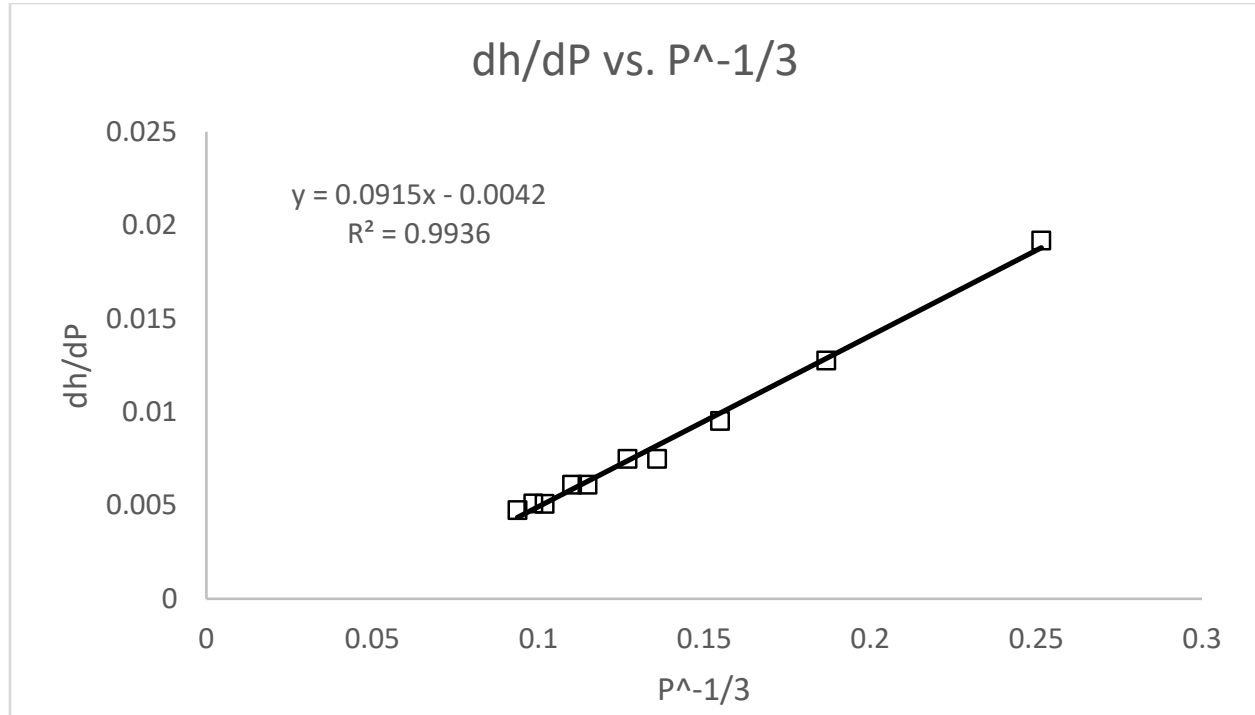

Figure A-6. FEA simulation of stiffness response of axisymmetric sphere $(D=10 \mathrm{~mm})$

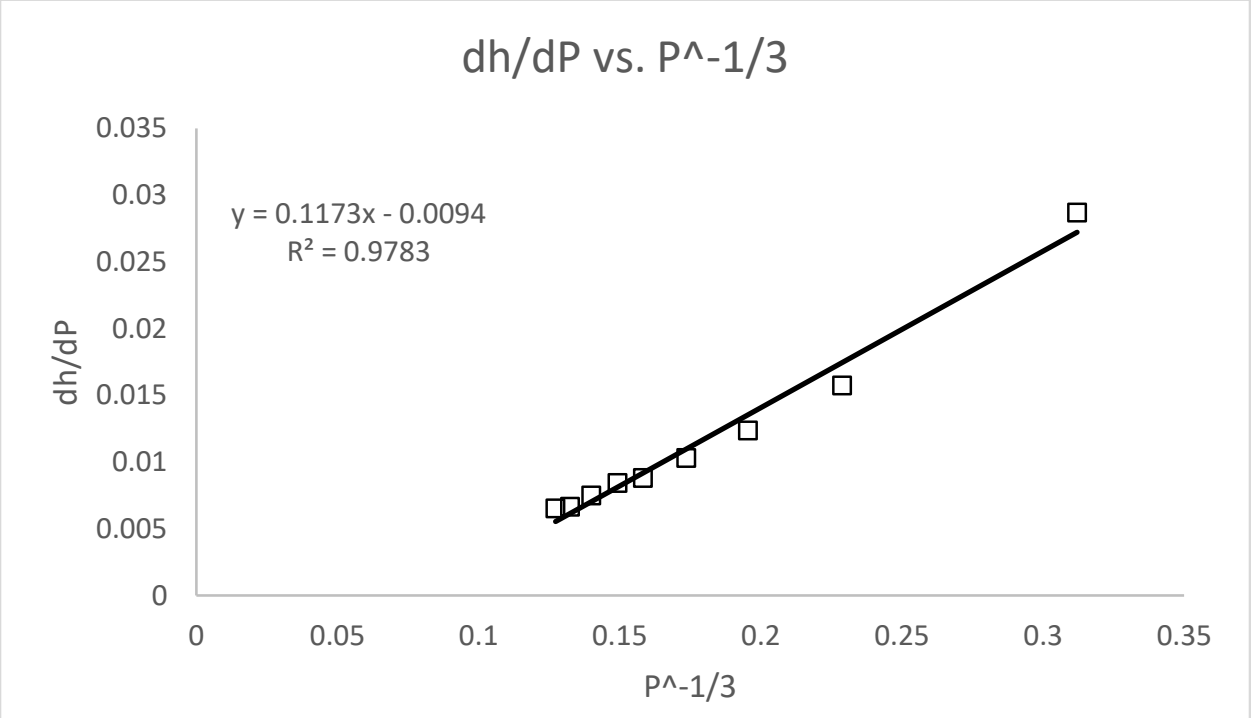

Figure A-7. FEA simulation of stiffness response of axisymmetric sphere $(D=5 \mathrm{~mm})$ 


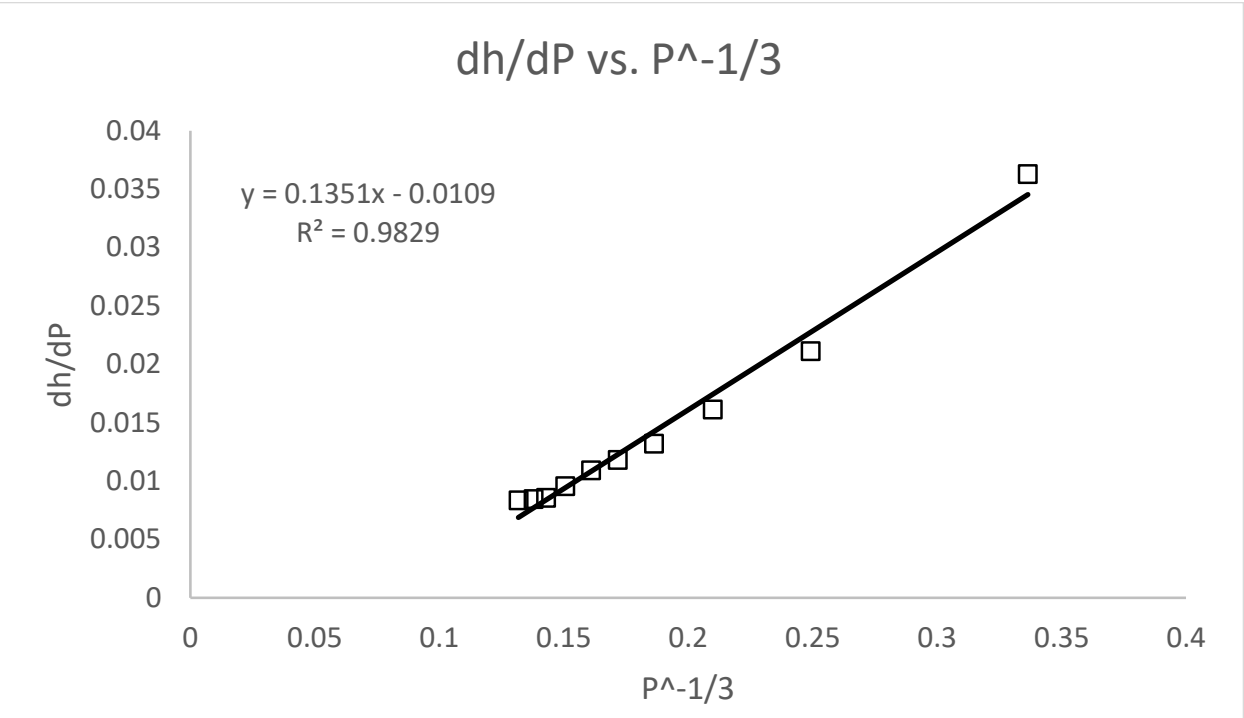

Figure A-8. FEA simulation of stiffness response of axisymmetric sphere $(D=3 \mathrm{~mm})$

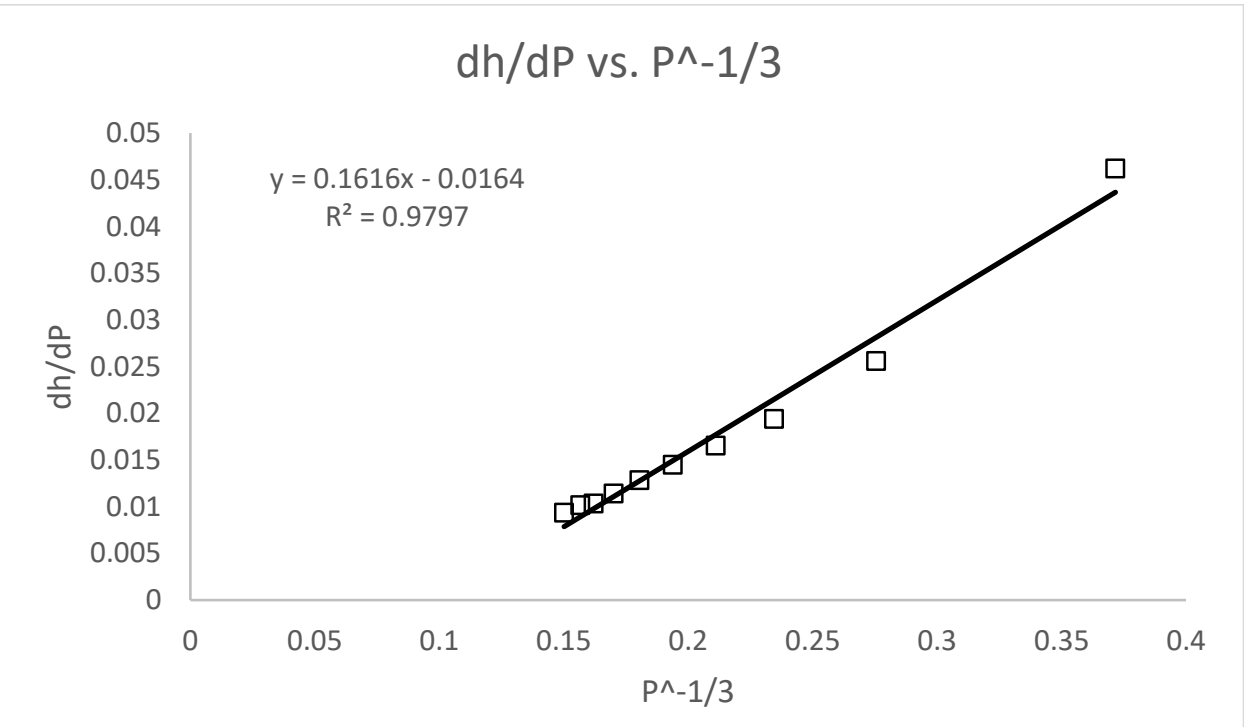

Figure A-9. FEA simulation of stiffness response of axisymmetric sphere ( $D=2 \mathrm{~mm})$ 


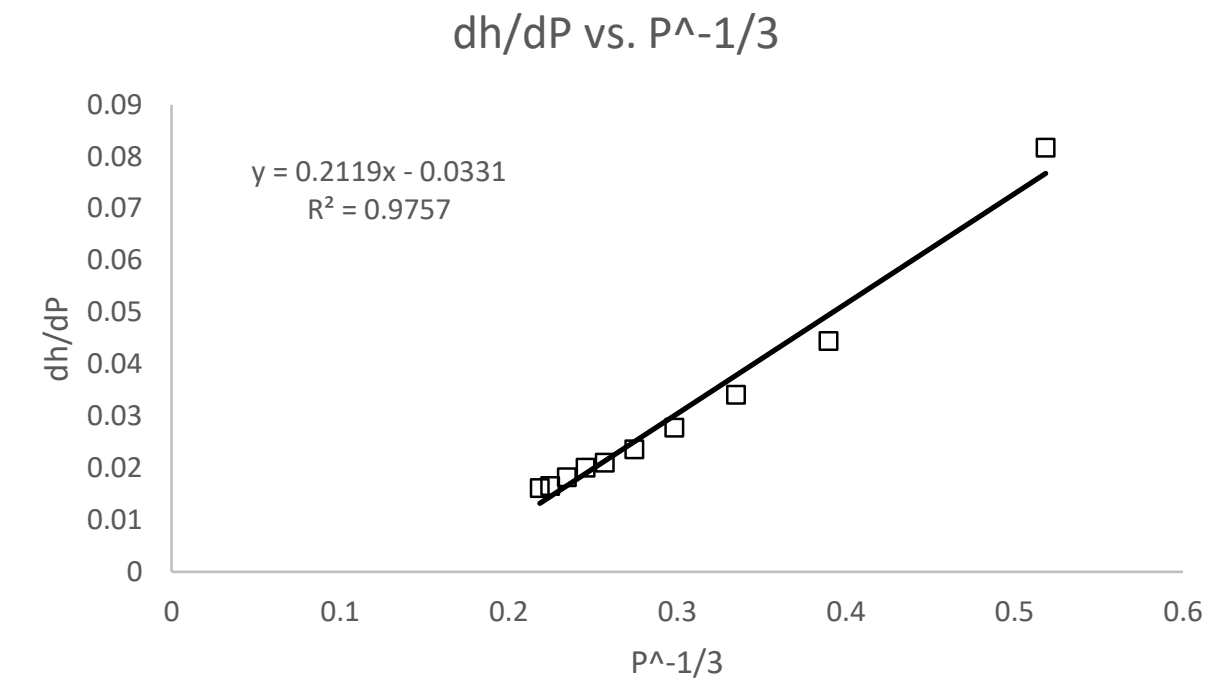

Figure A-10. FEA simulation of stiffness response of axisymmetric sphere $(D=1 \mathrm{~mm})$

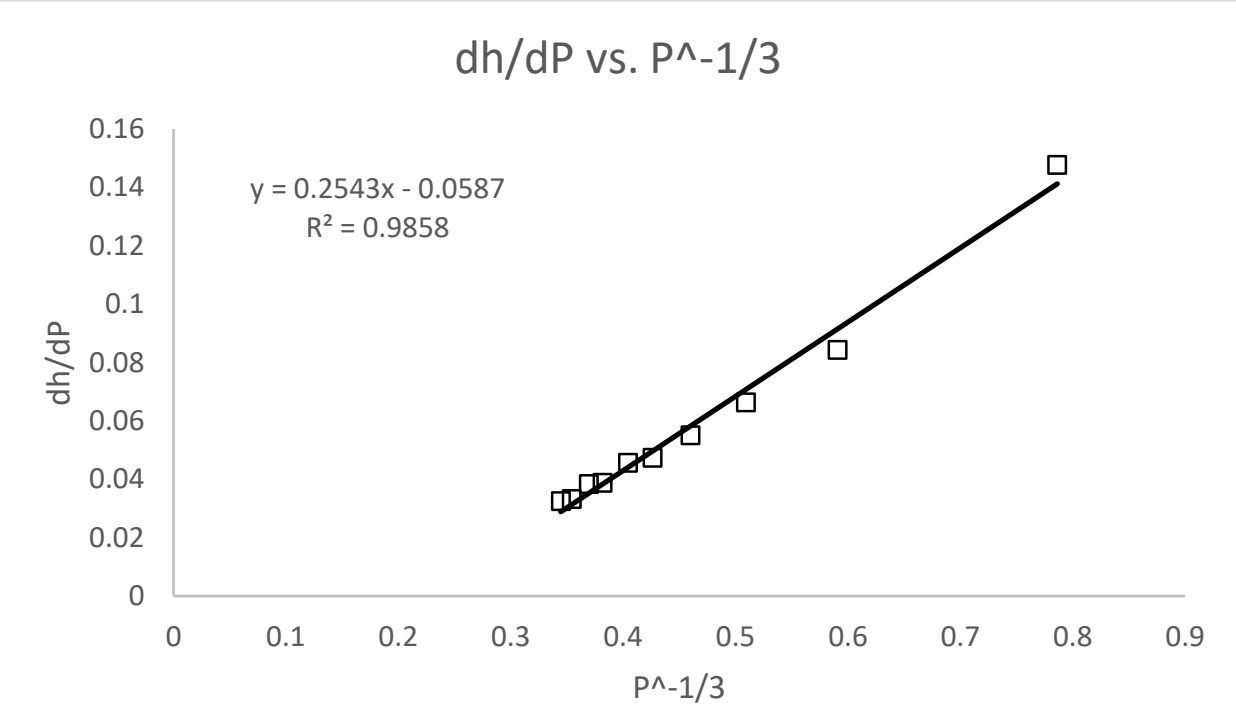

Figure A-11. FEA simulation of stiffness response of axisymmetric sphere ( $D=0.5 \mathrm{~mm})$

As shown, the simulation is capable of determining the stiffness response of spheres as small as $500 \mu \mathrm{m}$. Table A-1 summarizes the results, given the size of the sphere. It is evident there is no apparent relationship with the size of the sphere and the Young's Modulus. However, for powders in the lower micron scale, there is an expected relationship. 
Table A-1. FEA Simulation Results of sphere with various sizes.

\begin{tabular}{ccc} 
Size $(\mathrm{mm})$ & $\mathrm{E}_{\mathrm{R}}(\mathrm{GPa})$ & $\mathrm{E}(\mathrm{GPa})$ \\
\hline 10 & 208.72 & 195.68 \\
5 & 203.21 & 190.51 \\
3 & 212.23 & 198.97 \\
2 & 198.71 & 186.29 \\
1 & 187.17 & 174.47 \\
0.5 & 201.38 & 188.79
\end{tabular}

\section{A.3.2 Experimental Multiple Loading-Partial Unloading Micro-indentation}

Given the successful results of the FEA simulations shown above, experimental tests were conducted for verification. For proof on concept, Experimental testing was done on SS 316, Brass alloy 216, and Aluminum alloy 1100 spheres. Several diameters of the spheres were purchased and tested using the multiple loading-partial unloading indentation technique. Initially, a spherical indenter is used to indent the surface of the sample holder. A tiny indent is made to hold the SS ball in place during testing and the center of the spherical call align with the loading of the sample. Initial contact is made with the sample and indentation of $5 \mu \mathrm{m}$ and unload $2 \mu \mathrm{m}$ to for a total of 10 steps is done.

Table A-2. Experimental Results using Micro-Indentation Technique

\begin{tabular}{cccc} 
Material & Size $(\mathrm{mm})$ & $\mathrm{E}_{\mathrm{R}}(\mathrm{GPa})$ & $\mathrm{E}(\mathrm{GPa})$ \\
\hline $\mathrm{SS}$ & 5 & 64.57 & 65.612 \\
& 3 & 72.076 & 74.21 \\
Brass & 6.35 & 26.73 & 25.68 \\
& 3.18 & 29.76 & 28.7275 \\
\hline Aluminum & 7.94 & 26.58 & 24.13 \\
& 6.35 & 29.12 & 26.82 \\
\hline
\end{tabular}




\section{References}

[1] Wong, K. V., and Hernandez, A., 2012, "A Review of Additive Manufacturing," ISRN Mech. Eng., 2012, pp. 1-10.

[2] Huang, S. H., Liu, P., Mokasdar, A., and Hou, L., 2013, "Additive Manufacturing and Its Societal Impact: A Literature Review," Int. J. Adv. Manuf. Technol., 67(58), pp. 1191-1203.

[3] Spierings, A. B., Herres, N., and Levy, G., 2010, "Influence of the Particle Size Distribution on Surface Quality and Mechanical Properties in Additive Manufactured Stainless Steel Parts," 21st Annu. Int. Solid Free. Fabr. Symp. - An Addit. Manuf. Conf. SFF 2010, August 9, 2010 - August 11, 2010, pp. 397-406.

[4] Hao, L., Savalani, M. M., Zhang, Y., Tanner, K. E., Heath, R. J., and Harris, R. A., 2007, "Characterization of Selective Laser-Sintered Hydroxyapatite-Based Biocomposite Structures for Bone Replacement," Proc. R. Soc. A Math. Phys. Eng. Sci., 463(2084), pp. 1857-1869.

[5] Sutton, A. T., Kriewall, C. S., Leu, M. C., and Newkirk, J. W., 2016, "Powders for Additive Manufacturing Processes: Characterization Techniques and Effects on Part Properties," Solid Free. Fabr. Proc., pp. 1004-1030.

[6] Cooke, A., and Slotwinski, J., 2012, Properties of Metal Powders for Additive Manufacturing: A Review of the State of the Art of Metal Powder Property Testing.

[7] Kakinuma, Y., Mori, M., Oda, Y., Mori, T., Kashihara, M., Hansel, A., and Fujishima, M., 2016, "Influence of Metal Powder Characteristics on Product Quality with Directed Energy Deposition of Inconel 625," CIRP Ann. - Manuf. Technol., 65(1), pp. 209-212.

[8] Kakinuma, Y., Mori, M., Oda, Y., Mori, T., Kashihara, M., Hansel, A., and Fujishima, M., 2016, "Influence of Metal Powder Characteristics on Product Quality with Directed Energy Deposition of Inconel 625," CIRP Ann. - Manuf. Technol., 65(1), pp. 209-212.

[9] Kleinknecht, ronald a, 1973, "Notice Warning Concerning Copyright Restrictions," J. Am. Dent. Assoc., 86.

[10] Antony, L. V. M., and Reddy, R. G., 2003, "High-Purity Metals Overview Processes for Production of High-Purity Metal Powders."

[11] Anderson, J. E., Bool, L. E. I., Arnold, G. W., and Leger, C. B., 2003, "Hot Gas Atomization."

[12] Rieken, J. R., Anderson, I. E., Kramer, M. J., Odette, G. R., Stergar, E., and Haney, E., 2012, "Reactive Gas Atomization Processing for Fe-Based ODS Alloys," J. Nucl. Mater., 428, pp. 65-75.

[13] Chen, G., Zhao, S. Y., Tan, P., Wang, J., Xiang, C. S., and Tang, H. P., 2018, "A Comparative Study of Ti-6Al-4V Powders for Additive Manufacturing by Gas Atomization, Plasma Rotating Electrode Process and Plasma Atomization," Powder Technol., 333, pp. 38-46.

[14] "AMT Advanced Materials Technology GmbH - Gas-Atomization" [Online]. Available: https://www.amt-advanced-materials-technology.com/processing/gasatomization/. [Accessed: 13-May-2018].

[15] "Our Technology - AP\&amp;C" [Online]. Available: http://advancedpowders.com/plasma-atomization-technology/our-technology/. 
[Accessed: 13-May-2018].

[16] Benjamin, J. ., 1970, "Dispersion Strengthened Superalloys by Mechanical Alloying," Metall. Trans., 1(10), pp. 2943-2951.

[17] Weinbruch, S., Anastasiadis, A., Ortner, H. M., Martinz, H. P., and Wilharitz, P., 1999, "On the Mechanism of High Temperature Oxidation of ODS Superalloys: Significance of Yttrium Depletion Within the Oxide Scales," Oxid. Met., (51), pp. 111-128.

[18] Gilman, P. S., and Benjamin, J. ., 1983, "Mechanical Alloying," Annual Review of Materials Science, pp. 279-300.

[19] Bhadeshia, H. K. D. ., 1997, "Recrystallisation of Practical Mechanically Alloyed Iron-Base and Nickel-Base Superalloys," Mater. Sci. Eng. A, 1(223), pp. 64-77.

[20] Park, L. J., Ryu, H. J., Hong, S. H., and Kim, Y. G., 1998, "Microstructure and Mechanical Behavior of Mechanically Alloyed ODS Ni-Base Superalloy for Aerospace Gas Turbine Application," Adv. Perform. Mater.

[21] Chia, E., Kang, B. S., Zheng, M., Li, Y., and Chyu, M., 2018, "DEVELOPMENT OF ODS COATING FOR HIGH TEMPERATURE TURBINE COMPONENTS USING DED ADDITIVE MANUFACTURING," Proceeding of ASME Turbo Expo 2018, Oslo, Norway.

[22] Ma, L., Kang, B. S. J., Alvin, M. A., and Huang, C. C., 2014, "Characterization of Oxide-Dispersion-Strengthened (ODS) Alloy Powders Processed by MechanoChemical-Bonding (MCB) and Balling Milling (BM)," KONA Powder Part. J., 31(1), pp. 146-155.

[23] Mino, K., and Asakawa, K. J., 1987, "An Oxide Dispersion Strengthened NickelBase Superalloy with Excellent High Temperature Strength," 27(10), pp. 823-842.

[24] Yu, Y., Zhou, J., Chen, J., Zhou, H., Guo, C., and Guo, B., 2010, "Synthesis of Nanocrystalline Ni3Al by Mechanical Alloying and Its Microstructural Characterization," J. Alloys Compd., 498(1), pp. 107-112.

[25] Donachie, M. ., and Donachie, S. J., 2002, "Superalloys a Technical Guide," ASM Int. [Online]. Available: www.asminternational.org. [Accessed: 22-May-2019].

[26] Pollock, T. M., and Tin, S., 2006, "Nickel-Based Superalloys for Advanced Turbine Engines: Chemistry, Microstructure and Properties," J. Propuls. Power, 22(2), pp. 361-374.

[27] Srinivasan, D., and Al., E., 2006, "Strengthening Mechanisms (via Hardness Analysis) in Nanocrystalline Y2O3 and Al2O3 Dispersoids," 416(1-2), pp. 211218.

[28] Benfu, H., and er al., 2007, "Research and Development of New High Performance Powder Superalloys," (2), pp. 49-53.

[29] Suryanarayana, C., 2001, "Mechanical Alloying and Milling Mechanical Engineering," Prog. Mater. Sci., 46, pp. 1-184.

[30] Intrater, J., 2007, "Mechanical Alloying and Milling , C. Suryanarayana ," Mater. Manuf. Process., 22(6), pp. 790-791.

[31] Koch., C. S. and C. C., 2000, "Nanocrystalline Materials - Current Research and Future Directions," Hyperfine Interact., pp. 5-44.

[32] Choi, J. P., and Al., E., 2017, "Densification and Microstructural Investigation of Inconel 718 Parts Fabricated by Selective Lasr Melting," 310, pp. 60-66.

[33] Kantzos, C., Pauza, J., Cunningham, R., Narra, S. P., Beuth, J., and Rollett, A., 
2019, "An Investigation of Process Parameter Modifications on Additively Manufactured Inconel 718 Parts," J. Mater. Eng. Perform., 28(2), pp. 620-626.

[34] Zhang, F. L., Zhu, M., and Wang, C. Y., "Parameters Optimization in the Planetary Ball Milling of Nanostructured Tungsten Carbide/Cobalt Powder."

[35] Frazier, W. E. J. J. o. M. ., 2014, "Metal Additive Manufacturing: A Review," pp. 14-18.

[36] Li, R., 2010, "Densification Behavior of Has and Water Aromized 316L Stainless Steel Powder during Selevtive Laser Melting," 256(13), pp. 4350-4356.

[37] Pan, X., and Ma, X. J. ., 2004, "Phase Transformations in Nanocrystalline TiO2 Milled in Different Milling Atmospheres," 177(11), pp. 4098-2103.

[38] Ansell, G., and Lenel, F. J. A. M., 1960, "Criteria for Yielding of DispersionStrengthened Alloys," 8(9), pp. 612-616.

[39] Busby, 2009, "Economic Benefits of Advanced Materials in Nuclear Power Systems," 392(2), pp. 301-306.

[40] Tan, J. ., Wong, W. L. ., and Dalgarno, K. W. J. A. ., 2017, "An Overview of Powder Granulometry on Feedstock and Part Performance in the Selective Laser Melting Processs."

[41] Lu, L., and Lai, M. O., Mechanical Alloying.

[42] Enayati, M. H., Sadeghian, Z., Salehi, M., and Saidi, A., 2004, "The Effect of Milling Parameters on the Synthesis of Ni3AI Intermetallic Compound by Mechanical Alloying," Mater. Sci. Eng. A, 375-377(1-2 SPEC. ISS.), pp. 809-811.

[43] Stevens, E. L., and et al., 2017, "Variation of Hardness, Microstructure, and Laves Phase Distribution in Direct Laser Deposited Alloy 718 Cuboids," 119, pp. 188198.

[44] Huang, S., 2018, "Developemtn of Oxide-Dispersion Strengthened Nickel-Based Superalloy Powders for Additive Manufacturing," West Virginia University.

[45] Dinda, G., and Al., E., 2009, "Laser Aided Direct Metal Deposition of Inconel 625 Superalloy: Microstructural Evolution and Thermal Stability.," 509(1-2), pp. 98104.

[46] Jia, Q., and Gu, D. J., 2014, "Selective Laser Melting Additive Manufacturing of Inconel 718 Superalloy Parts: Densification, Microstructure and Properties.," 585, pp. 713-721.

[47] Liu, F., and Al., E., 2011, "Microstructure and Residual Stress of Laser Rapid Formed Inconel 718 Nickel-Based Superalloy," 43(1), pp. 208-213.

[48] Hertz, H., and Agnew, J. R., 1881, "On the Contact of Elastic Solids," Math, 92.

[49] Feng, C., Tannenbaum, J. M., Kang, B. S., and Alvin, M. A., 2010, "A Load-Based Multiple-Partial Unloading Micro-Indentation Technique for Mechanical Property Evaluation," Exp. Mech., 50(6), pp. 737-743.

[50] Bhattacharya, A. K., and Nix, W. D., 1988, "Finite Element SImulation of Indentation Experiments," Int. J. Solids Struct., 24(9), pp. 881-891.

[51] International, A., 2002, "Stainless Steel (SS)," Atlas of Stress-Strain Curves, C.A. Parker, V. Flint, and B. Sanders, eds., Library of Congress Cataloging-inPublication Data, Materials Park, OH, pp. 193-202. 